\title{
Resisting B-H Oxidative Addition: The Divergent Reactivity of the $o$-Carborane and Carba-closo-dodecaborate Ligand Substituents
}

\author{
AUTHORS \\ Jess Estrada, ${ }^{\dagger}$ Sarah E. Lee, ${ }^{\dagger}$ Scott McArthur, ${ }^{\dagger}$ Ahmad El-Hellani, ${ }^{\dagger}$ Fook S. Tham,${ }^{\dagger}$ and \\ Vincent Lavallo*† \\ $\dagger$ Department of Chemistry University of California Riverside, UCR Center for Catalysis, \\ Riverside, CA 92521 (USA).
}

\begin{abstract}
Here, we report a study of two isoelectronic $\operatorname{Ir}(\mathrm{I})$ complexes supported by different carboranyl phosphines, bearing either $o$-carborane or carba-closo-dodecaborate ligand substituents. The neutral $\operatorname{Ir}(\mathrm{I})$ complex containing the $o$-carboranyl phosphine ligand is not isolable and undergoes spontaneous B-H cyclometalation to afford an $\operatorname{Ir}(\mathrm{III})$ hydride. In contrast, the anionic $\operatorname{Ir}(\mathrm{I})$ complex supported by a phosphine with a carba-closo-dodecaborate ligand R-group is stable towards B-H activation. This divergent reactivity has important implications for the design of carborane containing ligands for catalysis. Both compounds are fully characterized by multinuclear NMR spectroscopy, HRMS spectrometry, and single crystal x-ray diffraction studies.
\end{abstract}

Keywords: carborane anion, carborane ligand, cyclometalation, carboranyl phosphine, boron cluster 


\section{Introduction}

The proliferation of contemporary homogenous catalytic methods is largely due to the availability of a diverse array of ligand architectures. Due to their shape and 3-dimensional aromaticity, icosahedral carboranes [1] are an interesting alternative to classical alkyl and aryl ligand substituents. Soon after the discovery of $o$-carborane [2] 1 (Fig 1, left) in 1963 a carboranyl phosphine ligand [3] and complex [4] were reported by Smith. Kumada [5] was the first to implement such ligands in homogenous catalysis, but these and all subsequent systems containing $o$-carborane $\mathbf{1}$

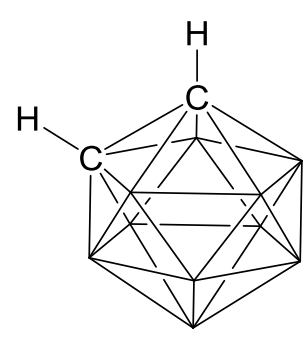

1
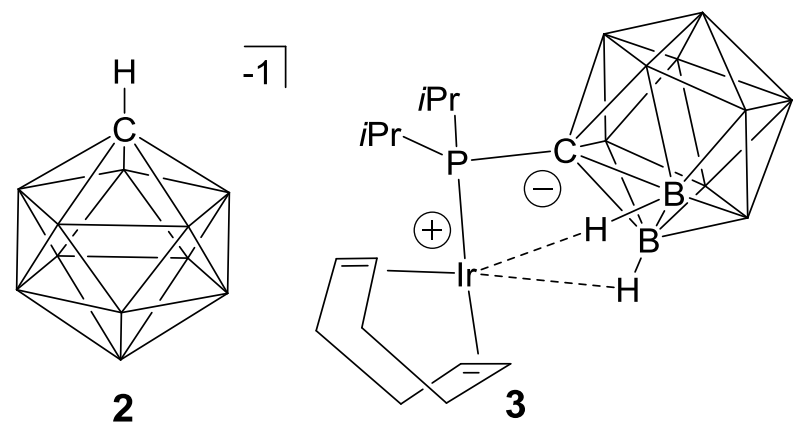

fragments have not yielded

Fig 1. o-Carborane 1, carba-closo-dodecaborate anion 2, and zwitterionic Ir complex 3. Unlabeled vertices $=\mathrm{B}-\mathrm{H}$

catalysts [6] that surpass the activity of systems supported by ubiquitous trialkyl or triaryl phosphines. Perhaps this non- competitive catalytic behavior is due to the decomposition of the cluster via well-known B-vertex extrusion $[1 \mathrm{~b}, 7]$ and B-H cyclometalation reactions. Hawthorne [8] was the first to observe intramolecular B-H oxidative addition of an $o$-carboranyl phosphine, as well as analogous intermolecular reactions with unsubstituted dicarbaborane icosahedra at an $\operatorname{Ir}(\mathrm{I})$ center.

In contrast to $o$-carborane $\mathbf{1}$, the isoelectronic and weakly coordinating carba-closododecaborate anion [1e] 2 (Fig 1, center) is not susceptible to B-vertex extrusion reactions and is renowned for its inert properties, particularly when polyhalogenated [1h, 9]. We recently reported the first utilization of the carba-closo-dodecaborate as a ligand substituent for a 
transition metal-based catalyst [10]. Notably, this zwitterionic Au(I) complex, featuring an anionic carboranyl phosphine, is far more active than all known systems for the hydroamination of alkynes. In addition, we reported an unusually stable pseudo-low coordinate zwitterionic $\operatorname{Ir}(\mathrm{I})$ compound 3 (Fig 1, right) that does not undergo spontaneous intramolecular B-H oxidative addition [11]. Although not identical, $\mathbf{3}$ is reminiscent of Hawthorne's unstable $\operatorname{Ir}(\mathrm{I})$ complexes [8] and its behavior suggests that ligands bearing a carba-closo-dodecaborate substituent [12] are inherently more resistant to B-H activation. Given our interest in catalyst design we are seeking stronger evidence that the carba-closo-dodecaborate ligand substituent has enhanced chemical stability towards B-H oxidative addition. Here, we report such a study by the preparation and analysis of two isoelectronic iridium (I) complexes containing phosphines with $o$-carborane and carba-closo-dodecaborate moieties, respectively. Where the former $o$-carborane containing complex is not isolable and spontaneously undergoes cyclometalation at one of the B-H vertices, the carba-closo-dodecaborate analogue is stable.

\section{Results and Discussion}

Reacting the known [13] $o$-carborane phosphine 4 with $1 / 2$ an equivalent of $(\mathrm{ClI}(\mathrm{COD}))_{2}$ in hexane solvent results in a color change from light orange to red orange and subsequent formation of a precipitate (Fig 2, next page). Based on Hawthorne's prior observations [8], it was predicted that the ensuing $\operatorname{Ir}(\mathrm{I})$ complex 5 would be unstable towards cyclometalation and produce the corresponding Ir(III) complex 6. Indeed, analysis of the precipitate by ${ }^{1} \mathrm{H}$ NMR spectroscopy shows clean formation of an iridium hydride at $-16.42 \mathrm{ppm}\left(\mathrm{d},{ }^{2} J_{\mathrm{P}-\mathrm{H}}=13.1 \mathrm{~Hz}\right)$, suggesting cyclometalation of the $o$-carborane cluster. The coupling constant is consistent with an iridium (III) hydride disposed cis to a phosphine (range ${ }^{2} J_{\mathrm{P}-\mathrm{H}}$ from $10-20 \mathrm{~Hz}$ ) [8]. In addition 

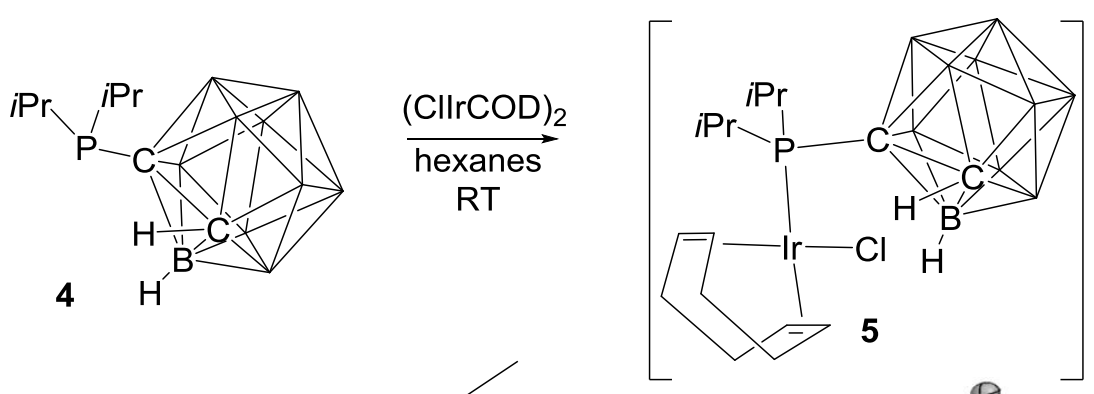

B3 Cyclometalated
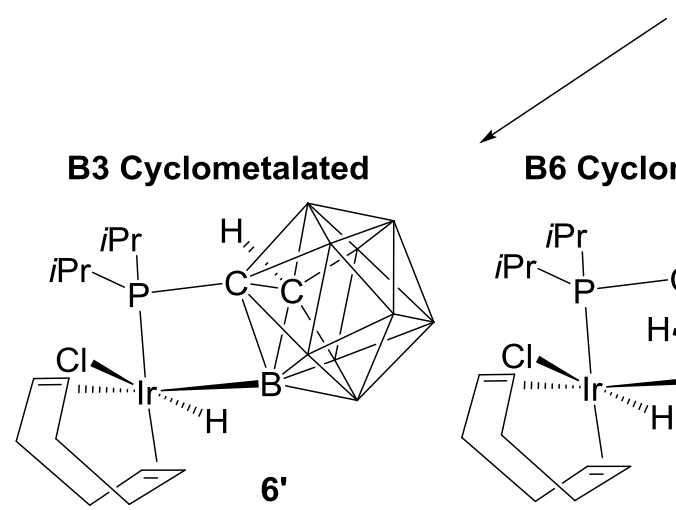

B6 Cyclometalated
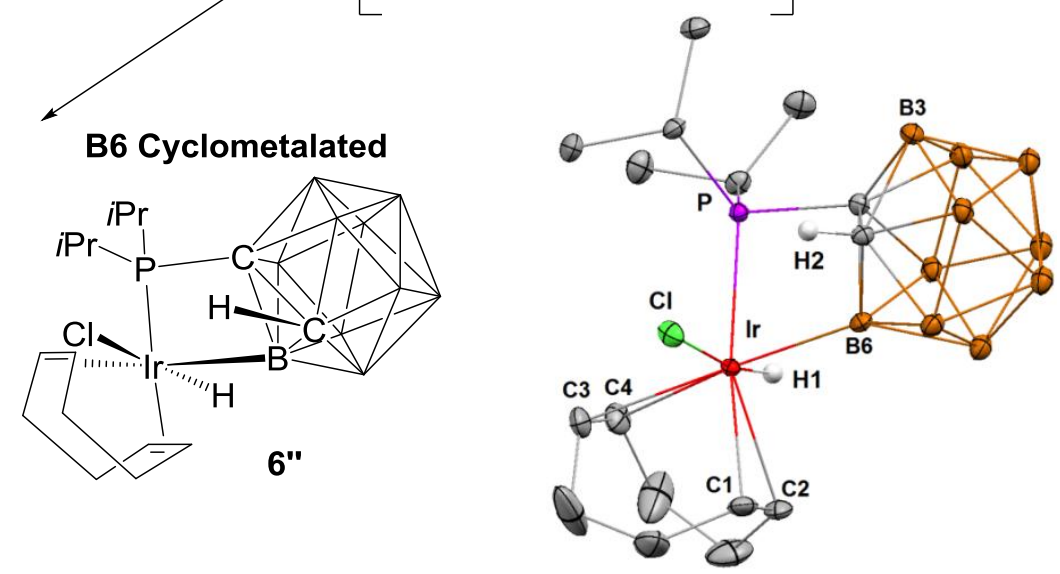

Fig 2. Synthesis of the cyclometalated Ir complexes 6. Solid-state structure of diastereomer 6". Color code: Grey $=\mathrm{C}$, white $=\mathrm{H}$, violet $=\mathrm{P}$, red $=\mathrm{Ir}$, brown $=\mathrm{B}$, green $=$ chlorine. Unlabeled vertices $=\mathrm{B}-\mathrm{H}$. Notable bond lengths $(\AA)$ : Ir-P = 2.320(5); Ir-B = 2.094(2); Ir-Cl = 2.498(5); C1-C2 = 1.384(3); C3-C4 = 1.362(3). Selected bond angles $\left({ }^{\circ}\right):$ B-Ir-P $=72.1 ; \mathrm{P}-\mathrm{Ir}-\mathrm{Cl}=90.2$.

to the expected ${ }^{1} \mathrm{H}$ signals for the phosphine $i$ Pr-groups and COD protons, two carborane $\mathrm{C}-\mathrm{H}$ resonances appear in a 4:1 ratio. The presence of two carborane $\mathrm{C}-\mathrm{H}$ signals suggests the formation of a diastereomeric mixture $\left(6^{\prime}, 6\right.$ '"), resulting from competitive B-H oxidative addition at the B3 and B6 positions (Fig 2). Such competitive cyclometalations have been observed previously [14] with other metals, and occur preferentially at B3/B6 positions rather than at B4/B5, since the B3/B6 boron atoms are more activated (attached to 2-carbon atoms). All other ligand ${ }^{1} \mathrm{H}$ resonances from the two diastereomers 6 ' and 6 '" are superimposed by coincidence. Analysis of the ${ }^{31} \mathrm{P}$ NMR spectra also shows a 4:1 mixture of compounds and is in agreement with our assignment. The ${ }^{11} \mathrm{~B}$ NMR spectrum is uninstructive due to the multitude of broad overlapping resonances, which cannot be resolved. However, the solution and solid-state 
infrared spectra of complexes 6 show bands typical for B-H stretches of $o$-carborane (2460-2625 $\mathrm{cm}^{-1}$ ), as well as the presence of an Ir-H absorbance at $2223 \mathrm{~cm}^{-1}$ [8].

A single-crystal X-ray diffraction study of one of the diastereomers 6" confirms that cyclometalation occurs adjacent to the two carborane carbon atoms (Figure 2, bottom right). Compound 6" adopts a distorted octahedral geometry with the hydride, which was located from the difference electron density map, cis to the phosphine ligand. The Ir-P, Ir-B and Ir-Cl bond lengths are 2.320(5), 2.094(2) and 2.498(5) $\AA$, respectively. The cyclooctadiene C-C double bond lengths trans to the phosphine $(\mathrm{C} 1-\mathrm{C} 2=1.384(3) \AA)$ and metalated B-vertex $(\mathrm{C} 3-\mathrm{C} 4=$ 1.362(3) Å) are in the typical range (1.35-1.44) for olefins coordinated to Ir [15].

We next turned our attention to preparing a complex bearing a phosphine with a carbacloso-dodecaborate substituent, which is isoelectronic with the unstable complex $\mathbf{5}$. We predicted that treating the previously reported [11] zwitterion $\mathbf{3}$ with a nucleophilic chloride source should produce the anionic isoelectronic complex 7 (Fig 3). Thus,
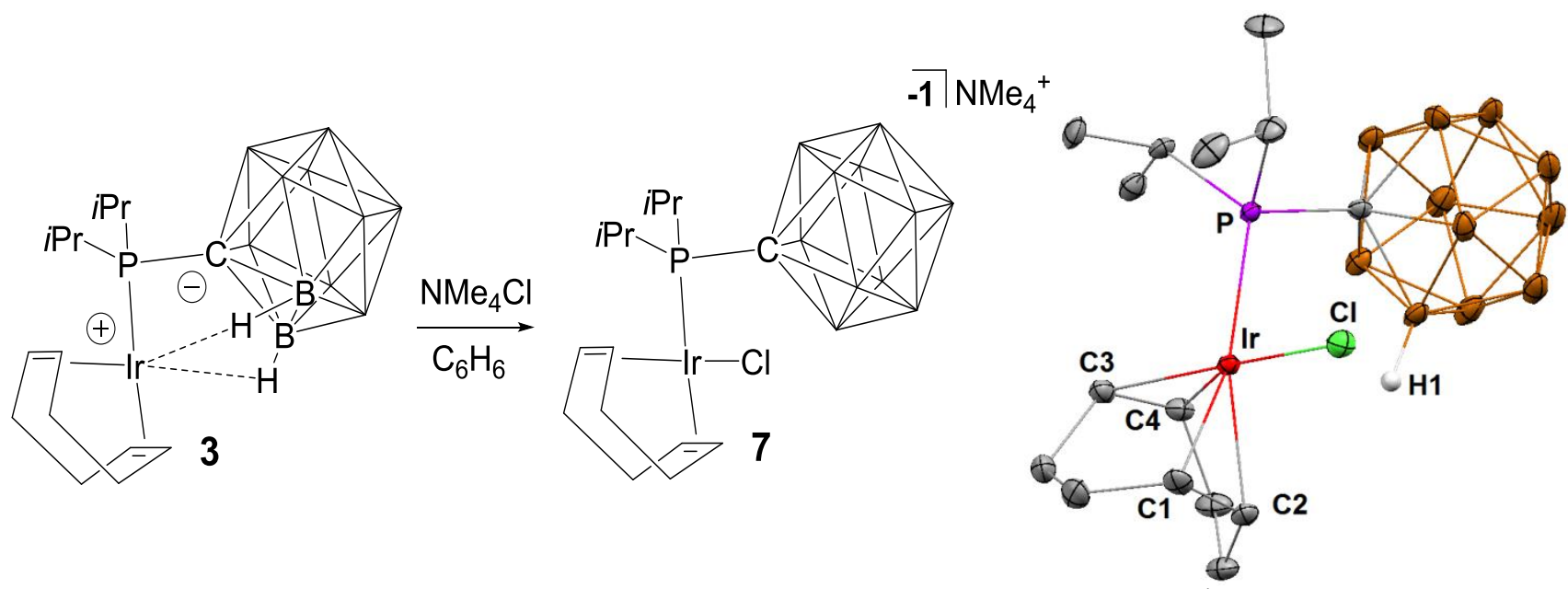

Fig 3. Synthesis of anionic $\operatorname{Ir}(\mathrm{I})$ complex 7. Solid-state structure of 7 (note: most hydrogens and $\mathrm{NMe}_{4}{ }^{+}$countercation omitted for clarity). Color code: grey $=\mathrm{C}$, white $=\mathrm{H}$, violet $=\mathrm{P}$, red $=\mathrm{Ir}$, brown $=\mathrm{B}$, green $=\mathrm{Cl}$. Unlabeled vertices $=\mathrm{B}$ $H$. Notable bond lengths $(\AA)$ : Ir-P = 2.371(7); Ir-Cl = 2.394(8); C1-C2 = 1.401(5); C3-C4 = 1.422(5). Selected bond angle $\left({ }^{\circ}\right)$ : $\mathrm{P}-\mathrm{Ir}-\mathrm{Cl}=91.4$. 
treatment of solution of 3 dissolved in $\mathrm{C}_{6} \mathrm{H}_{5} \mathrm{~F}$ with five equivalents of $\mathrm{NMe}_{4} \mathrm{Cl}$ resulted in the formation of an orange precipitate 7. Analysis of the precipitate by ${ }^{31} \mathrm{P}$ NMR shows the formation of a single new product 7 (s, $32.95 \mathrm{ppm})$. Importantly, the ${ }^{1} \mathrm{H}$ NMR spectrum of 7 shows no Ir-H resonance, suggesting that the carba-closo-dodecaborate substituent is not cyclometalated. Moreover, as indicated by ${ }^{11} \mathrm{~B}$ NMR spectroscopy (three resonances 1:5:5 ratio; 5:5 overlapping) the local $\mathrm{C}_{5 \mathrm{v}}$ symmetry of the cluster is retained, confirming that cyclometalation has not occurred. In addition, solution I.R. shows no Ir-H absorbance, ruling out the possibility of a rapid reversible cyclometalation process faster than the NMR time scale.

A single-crystal X-ray diffraction structure confirms the identity of the square planar Ir (I) complex 7 (Fig 3, previous page). While the Ir-P bond length $(2.371(7) \AA$ ) is similar to complex 6, the $\mathrm{Ir}-\mathrm{Cl}(2.394(8) \AA)$ and olefin bond lengths $(\mathrm{C} 1-\mathrm{C} 2=1.401(5) \AA, \mathrm{C} 3-\mathrm{C} 4=$ 1.422(5) $\AA$ ) are comparatively contracted and elongated, respectively. These observations can be explained by the decrease in coordination number and oxidation state, which allows for stronger M-L $\sigma$ and $\pi$ interactions. The closest B-H approach to the Ir center is 2.83 (Ir-H1) which is outside the range of typical Ir B-H agostic interactions. For comparison, the zwitterionic precursor 3 displays two strong agostic interactions with the metal center $(\mathrm{Ir}-\mathrm{H}$ distances $=$ 1.93(4) and 1.92(4) А) [11].

\section{Summary and Conclusion}

The study above provides strong evidence that the carba-closo-dodecaborate ligand substituent is far less susceptible to intramolecular B-H oxidative addition reactions compared to its neutral cousin, $o$-carborane. This observation is important since it supports the notion that $o$ carborane may not be a suitable ligand substituent for catalysts that mediate reactions involving 
oxidative addition/reductive elimination sequences. In addition, the ability of the carba-closododecaborate ligand R-group to resist intramolecular B-H oxidative addition suggests that ligands containing this group should be useful for a variety of catalytic processes.

\section{Experimental}

Unless otherwise stated, all manipulations were carried out using standard Schlenk or glovebox techniques $\left(\mathrm{O}_{2}, \mathrm{H}_{2} \mathrm{O}<1 \mathrm{ppm}\right)$ under an argon or dinitrogen atmosphere. The iridium complex 3 and 1-diisopropylphosphino-1,2-dicarba-closo-dodecaborane ligand $\mathbf{4}$ were prepared according to literature $[11,13]$. Solvents were dried on $\mathrm{NaK}, \mathrm{K}$, or $\mathrm{CaH}_{2}$ and distilled under argon before use. Reagents were purchased from commercial vendors and used without further purification.NMR spectra were recorded on Bruker Avance $300 \mathrm{MHz}$, Varian Inova $300 \mathrm{MHz}$, Varian Inova $400 \mathrm{MHz}$, or Varian Inova $500 \mathrm{MHz}$ spectrometers. Standard Varian pulse sequences, gCOSY and gHSQC were used for the COSY and HSQC experiments. Both are standard Varian pulse sequences that accompany the VnmrJ 2.2D software. ${ }^{1} \mathrm{H}$ NMR and ${ }^{13} \mathrm{C}$ NMR chemical shifts were referenced to residual solvent. ${ }^{11}$ B NMR chemical shifts were externally referenced to $\mathrm{BF}_{3} \mathrm{OEt}_{2} .{ }^{31} \mathrm{P}$ NMR chemical shifts were externally referenced to $80 \%$ $\mathrm{H}_{3} \mathrm{PO}_{4}$ in $\mathrm{H}_{2} \mathrm{O}$. HRMS was recorded on Agilent Technologies 6210 (time of flight LC/MS) using ESI technique. IR spectra were recorded on Attenuated Reflectance (ATR) and were run on ABB MB3000 spectrometer in the $525-4000 \mathrm{~cm}^{-1}$ frequency range using a diamond crystal.

Synthesis of complexes $6 \operatorname{Ir}(\mathrm{COD}) \mathrm{Cl}\left[\mathrm{P}(\mathrm{iPr})_{2} \mathrm{C}_{2} B_{10} \mathrm{H}_{11}\right]$ (mixture of two diastereomers)

A solution of $[\operatorname{Ir}(\mathrm{COD}) \mathrm{Cl}]_{2}(30 \mathrm{mg}, 0.089 \mathrm{mmol})$ in hexane $(6 \mathrm{~mL})$ was added to a hexane solution of 1-diisopropylphosphino-1,2-dicarba-closo-dodecaborane 4 (23.2 mg, $0.089 \mathrm{mmol})$ and allowed to stir overnight. The resulting precipitate was filtered and washed with hexane $(3 \mathrm{x}$ $1 \mathrm{~mL}$ ) to afford 6 as a vermilion solid in $95 \%$ yield $(50.5 \mathrm{mg}, 0.084 \mathrm{mmol}) .{ }^{1} \mathrm{H}$ NMR (300 MHz, 
$\left.\mathrm{C}_{6} \mathrm{D}_{6}, 25^{\circ} \mathrm{C}\right): \delta=5.87$ (bs, $\left.1 \mathrm{H}, \mathrm{C}-\mathrm{H}_{\text {carborane minor isomer }}\right), 5.75$ (bs, $\left.1 \mathrm{H}, \mathrm{C}-\mathrm{H}_{\text {carborane major isomer }}\right), 5.15$ (m, 1H, $\left.\mathrm{CH}_{\mathrm{COD}}\right), 5.03\left(\mathrm{~m}, 1 \mathrm{H}, \mathrm{CH}_{\mathrm{COD}}\right), 4.86\left(\mathrm{~m}, 1 \mathrm{H}, \mathrm{CH}_{\mathrm{COD}}\right), 4.55\left(\mathrm{~m}, 1 \mathrm{H}, \mathrm{CH}_{\mathrm{COD}}\right), 2.95(\mathrm{~m}, 1 \mathrm{H}$, $\left.\mathrm{CH}_{2}\right), 2.59(\mathrm{~m}, 1 \mathrm{H}, \mathrm{CH}), 2.53\left(\mathrm{~m}, 1 \mathrm{H}, \mathrm{CH}_{2}\right), 2.26(\mathrm{~m}, 1 \mathrm{H}, \mathrm{CH}), 2.08\left(\mathrm{~m}, 1 \mathrm{H}, \mathrm{CH}_{2}\right), 1.89(\mathrm{~m}, 2 \mathrm{H}$, $\left.\mathrm{CH}_{2}\right), 1.81\left(\mathrm{~m}, 1 \mathrm{H}, \mathrm{CH}_{2}\right), 1.73\left(\mathrm{~m}, 1 \mathrm{H}, \mathrm{CH}_{2}\right), 1.63\left(\mathrm{~m}, 1 \mathrm{H}, \mathrm{CH}_{2}\right), 0.99\left(\mathrm{dd},{ }^{3} \mathrm{~J}(\mathrm{P}-\mathrm{H})=15.7,{ }^{3} \mathrm{~J}(\mathrm{H}-\right.$ $\left.\mathrm{H})=7.5 \mathrm{~Hz}, 3 \mathrm{H}, \mathrm{CH}_{3}\right), 0.94\left(\mathrm{dd},{ }^{3} \mathrm{~J}(\mathrm{P}-\mathrm{H})=19.5,{ }^{3} \mathrm{~J}(\mathrm{H}-\mathrm{H})=7.4 \mathrm{~Hz}, 3 \mathrm{H}, \mathrm{CH}_{3}\right), 0.85\left(\mathrm{dd},{ }^{3} \mathrm{~J}(\mathrm{P}-\mathrm{H})=\right.$ $\left.16.9,{ }^{3} \mathrm{~J}(\mathrm{H}-\mathrm{H})=7.5 \mathrm{~Hz}, 3 \mathrm{H}, \mathrm{CH}_{3}\right), 0.75\left(\mathrm{dd},{ }^{3} \mathrm{~J}(\mathrm{P}-\mathrm{H})=12.0,{ }^{3} \mathrm{~J}(\mathrm{H}-\mathrm{H})=7.3 \mathrm{~Hz}, 3 \mathrm{H}, \mathrm{CH}_{3}\right),-16.42(\mathrm{~d}$, $\left.{ }^{2} \mathrm{~J}(\mathrm{P}-\mathrm{H})=13.1 \mathrm{~Hz}, 1 \mathrm{H}, \mathrm{Ir}-\mathrm{H}\right) ;{ }^{13} \mathrm{C}\left\{{ }^{1} \mathrm{H}\right\} \mathrm{NMR}\left(126 \mathrm{MHz}, \mathrm{C}_{6} \mathrm{D}_{6}, 25{ }^{\circ} \mathrm{C}\right): \delta=104.7\left(\mathrm{CH}_{\mathrm{COD}}\right), 94.6$ $\left(\mathrm{CH}_{\mathrm{COD}}\right), 86.9\left(\mathrm{~d},{ }^{2} \mathrm{~J}(\mathrm{P}-\mathrm{C})=17.4 \mathrm{~Hz}, \mathrm{CH}_{\mathrm{COD}}\right), 82.0\left(\mathrm{CH}_{\mathrm{COD}}\right), 64.7\left(\mathrm{CH}_{\text {carborane }}\right) 32.8\left(\mathrm{CH}_{2}\right), 32.2$ $\left(\mathrm{d},{ }^{1} \mathrm{~J}(\mathrm{P}-\mathrm{C})=19.8 \mathrm{~Hz}, \mathrm{CH}\right), 32.1\left(\mathrm{CH}_{2}\right), 31.3\left(\mathrm{CH}_{2}\right), 27.7\left(\mathrm{CH}_{2}\right), 24.3\left(\mathrm{~d},{ }^{1} \mathrm{~J}(\mathrm{P}-\mathrm{C})=12.9 \mathrm{~Hz}, \mathrm{PCH}\right)$, $21.6\left(\mathrm{CH}_{3}\right), 20.2\left(\mathrm{CH}_{3}\right), 18.4\left(\mathrm{~d},{ }^{2} \mathrm{~J}(\mathrm{P}-\mathrm{C})=6.2 \mathrm{~Hz}, \mathrm{CH}_{3}\right), 18.4\left(\mathrm{~d},{ }^{2} \mathrm{~J}(\mathrm{P}-\mathrm{C})=7.3 \mathrm{~Hz}, \mathrm{CH}_{3}\right) ;{ }^{31} \mathrm{P}\left\{{ }^{1} \mathrm{H}\right\}$ NMR (162 MHz, $\left.\mathrm{C}_{6} \mathrm{D}_{6}, 25{ }^{\circ} \mathrm{C}\right): \delta=43.0,20.9 \mathrm{ppm} ;{ }^{11} \mathrm{~B}\left\{{ }^{1} \mathrm{H}\right\} \operatorname{NMR}\left(96 \mathrm{MHz}, \mathrm{C}_{6} \mathrm{D}_{6}, 25{ }^{\circ} \mathrm{C}\right): \delta=$ $2.8, \quad 0.0,-3.1,-4.3,-5.7,-6.6,-7.8,-9.6,-18.0$ (B-Ir) ppm. HRMS: calculated [M-] $\left\{\mathrm{C}_{16} \mathrm{H}_{37} \mathrm{~B}_{10} \mathrm{ClIrP}\right\}: 596.2949 \mathrm{~m} / z$; Found [M-] $\left\{\mathrm{C}_{16} \mathrm{H}_{37} \mathrm{~B}_{10} \mathrm{ClIrP}\right\}: 596.2915 \mathrm{~m} / \mathrm{z}$. (Note: the resonance for the carborane carbon attached to $\mathrm{P}$ is not observable in the ${ }^{13} \mathrm{C}$ NMR spectrum.)

Synthesis of complex $7 \operatorname{Ir}(\mathrm{COD}) \mathrm{Cl}\left[\mathrm{P}(\mathrm{iPr})_{2} \mathrm{CB}_{11} \mathrm{H}_{11}\right]\left[\mathrm{N}_{(}\left(\mathrm{CH}_{3}\right)_{4}\right]$

Complex 3 (50 mg, $0.089 \mathrm{mmol}$ ) was dissolved in a solution of fluorobenzene and tetramethylammonium chloride ( $48.95 \mathrm{mg}, 0.45 \mathrm{mmol}$ ) was added to the solution and stirred overnight. The resulting precipitate was filtered and washed with fluorobenzene $(2 \times 1 \mathrm{~mL})$, and hexane $(2 \times 1 \mathrm{~mL})$ to afford 7 as a light red solid in $95 \%$ yield $(50.5 \mathrm{mg}, 0.085)$. Crystals suitable for a single crystal $\mathrm{x}$-ray diffraction study were obtained by layering a tetrahydrofuran solution with hexanes. ${ }^{1} \mathrm{H}$ NMR $\left(500 \mathrm{MHz}, \mathrm{THF}-d_{8}, 25{ }^{\circ} \mathrm{C}\right): \delta=4.55$ (bs, $\left.2 \mathrm{H}, \mathrm{CH}_{\mathrm{COD}}\right), 4.09$ (bs, $2 \mathrm{H}$, $\left.\mathrm{CH}_{\mathrm{COD}}\right), 3.27\left(\mathrm{~s}, 12 \mathrm{H}, \mathrm{N}\left(\mathrm{CH}_{3}\right)_{4}{ }^{+}\right), 2.86(\mathrm{~m}, 2 \mathrm{H}, \mathrm{CH}), 2.15$ (bm, 4H, $\left.\mathrm{CH}_{2}\right), 2.03$ (bm, 4H, $\mathrm{CH}_{2}$ ), $1.48\left(\mathrm{dd},{ }^{3} \mathrm{~J}(\mathrm{P}-\mathrm{H})=15.7,{ }^{3} \mathrm{~J}(\mathrm{H}-\mathrm{H})=6.9 \mathrm{~Hz}, 6 \mathrm{H}, \mathrm{CH}_{3}\right), 1.33\left(\mathrm{dd},{ }^{3} \mathrm{~J}(\mathrm{P}-\mathrm{H})=12.9,{ }^{3} \mathrm{~J}(\mathrm{H}-\mathrm{H})=6.9 \mathrm{~Hz}\right.$, 
$\left.6 \mathrm{H}, \mathrm{CH}_{3}\right) \mathrm{ppm} ;{ }^{31} \mathrm{P}\left\{{ }^{1} \mathrm{H}\right\}$ NMR $\left(300 \mathrm{MHz}, \mathrm{THF}-d_{8}, 25{ }^{\circ} \mathrm{C}\right): \delta=32.9 \mathrm{ppm} ;{ }^{11} \mathrm{~B}\left\{{ }^{1} \mathrm{H}\right\}$ NMR $(300$

MHz, THF- $\left.d_{8}, 25{ }^{\circ} \mathrm{C}\right): \delta=0.8,-9.0,-9.7 \mathrm{ppm}$. HRMS: calculated [M-] $\left\{\mathrm{C}_{15} \mathrm{H}_{37} \mathrm{~B}_{11} \mathrm{ClIrP}\right\}$ :

$595.3047 \mathrm{~m} / \mathrm{z}$; Found [M-] $\left\{\mathrm{C}_{15} \mathrm{H}_{37} \mathrm{~B}_{11} \mathrm{ClIrP}\right\}: 595.3061 \mathrm{~m} / \mathrm{z}$.

\section{Author Information}

Corresponding Author

*Email: vincent.lavallo@ucr.edu.

\section{Author Contributions}

The manuscript was written through contributions of all authors. All authors have given approval to the final version of the manuscript. These authors contributed equally.

\section{Notes}

The authors declare no competing financial interests.

\section{Acknowledgments}

We are grateful to NSF (award \# CHE 1455348) and the ACS PRF (award \# 52255-DNI3) for financial support of this work. J.E. was supported by a US Department of Education GAANN Award (no. P200A120170).

\section{References}

[1.] For recent reviews on carborane clusters, see: (a) R.N. Grimes, Dalton Trans. 44 (2015) 59395956; (b) A.R. Popescu, F. Teixidor, C. Viñas, Coord. Chem. Rev. 269 (2014) 54-84; (c) A.M. Spokoyny, Pure Appl. Chem. 85 (2013) 903-919; (d) D. Olid, R. Núñez, C. Viñas, F. Teixidor, Chem. Soc. Rev. 42 (2013) 3318; (e) C. Douvris, J. Michl, Chem. Rev. 113 (2013) PR179-PR233; (f) P. Farras, E.J. Juarez-Perez, M. Lepsik, R. Luque, R. Núñez, F. Teixidor, Chem. Soc. Rev. 41 (2012) 3445-3463; (g) M. Scholz, E. Hey-Hawkins, Chem. Rev. 111 (2011) 7035-7062; (h) C.A Reed, Acc. Chem. Res. 43 (2009) 121-128; (i) Y. Li, P.J. Carroll, L.G. Sneddon, Inorg. Chem. 47 (2008) 91939202; For recent discussions on the 3-dimensional aromaticity of carborane clusters, see: (j) J. Poater, M. Solà, C. Viñas, F. Teixidor, Angew. Chem. Int. Ed. 53 (2014) 12191-12195; (k) M. Asay, C.E. Kefalidis, J. Estrada, D.S. Weinberger, J. Wright, C.E. Moore, A.L. Rheingold, L. Maron, V. Lavallo, Angew. Chem. Int. Ed. 52 (2013) 11560-11563; (I) J.H. Wright II, C.E. Kefalidis, F.S. Tham, L. Maron, V. Lavallo, Inorg. Chem. 52 (2013) 6223-6229.

[2.] T.L. Heying, J.W. Ager, S.L Clark, D.J. Mangold, H.L. Goldstein, M. Hillman, R.J Polak, J.W. Szymanski, Inorg. Chem. 2 (1963) 1089-1092.

[3.] H.D. Smith, T.A. Knowles, H. Schroeder, Inorg. Chem. 4 (1965) 107-111.

[4.] H.D. Smith, J. Am. Chem. Soc. 87 (1965) 1817-1818.

[5.] K. Tamao, Y. Kiso, K. Sumitani, M.J. Kumada, J. Am. Chem. Soc. 94 (1972) 9268-9269. 
[6.] Recently Bourissou and coworkers have published a series of papers descibing unprecedented stoichiometric oxidative addition reactions with $\mathrm{Au}(\mathrm{I})$ complexes supported chelating ocarboranyl diphosphines: (a) M. Joost, L. Estévez, K. Miqueu, A. Amgoune, D. Bourissou, Angew. Chem. Int. Ed. (2015) DOI: 10.1002/anie.201500458; (b) M. Joost, A. Zeineddine, L. Estévez, S. Mallet-Ladeira, K. Miqueu, A. Amgoune, D. Bourissou, J. Am. Chem. Soc. 136 (2014) 1465414657; (c) M. Joost, L. Estévez, S. Mallet-Ladeira, K. Miqueu, A. Amgoune, D. Bourissou, Angew. Chem. Int. Ed. 53 (2014) 14512-14516.

[7.] For examples of ligand framework utilizing functionalized nido-dicarbaboranes, see: (a) A.-R. Popescu, A. Laromaine, F. Teixidor, R. Sillanpää, R. Kivekäs, J.I. Llambias, C. Viñas, Chem. Eur. J. 17 (2011) 4429-4443; (b) P. Farràs, F. Teixidor, I. Rojo, R. Kivekäs, R. Sillanpää, P. GonzálezCardoso, C. Viñas, J. Am. Chem. Soc. 133 (2011) 16537-16552; (c) P. Farràs, D. Olid-Britos, C. Viñas, F. Teixidor, Eur. J. Inorg. Chem. 2011 (2011) 2525-2532; (d) F. Teixidor, R. Núñez, M.A. Flores, A. Demonceau, C. Viñas, J. Organomet. Chem. 614-615 (2000) 48-56; (e) F. Teixidor, M.A. Flores, C. Viñas, R. Sillanpää, R. Kivekäs, J. Am. Chem. Soc. 122 (2000) 1963-1973; (f) F. Teixidor, M.A. Flores, C. Viñas, R. Kivekäs, R. Sillanpää, Organometallics 17 (1998) 4675-4679; (g) C. Viñas, R. Núñez, F. Teixidor, R. Kivekäs, R. Sillanpää, Organometallics 15 (1996) 3850-3858; (h) F. Teixidor, J.A. Ayllòn, C. Viñas, R. Kivekäs, R. Sillanpää, J. Casabò, J. Organomet. Chem. 483 (1994) 153-157; (i) F. Teixidor, J.A. Ayllon, C. Viñas, R. Kivekäs, R. Sillanpää, J. Casabo, Inorg. Chem. 33 (1994) 1756-1761; (j) F. Teixidor, J.A. Ayllon, C. Viñas, R. Kivekäs, R. Sillanpää, J. Casabo, Organometallics 13 (1994) 2751-2760; (k) F. Teixidor, C. Viñas, M. Mar Abad, M. Lopez, J. Casabo, Organometallics 12 (1993) 3766-3768; (I) F. Teixidor, J.A. Ayllon, C. Viñas, R. Kivekäs, R. Sillanpää, J. Casabo, J. Chem. Soc., Chem. Commun. (1992) 1281-1282.

[8.] E.L. Hoel, M.F. Hawthorne, J. Am. Chem. Soc. 97 (1975) 6388-6395.

[9.] For recent examples on the use of inert polyhalogenated carborane anions, see: (a) R.J. Wehmschulte, K.K. Laali, G.L. Borosky, D.R. Powell, Organometallics 33 (2014) 2146-2147; (b) R.J. Wehmschulte, M. Saleh, D.R. Powell, Organometallics 32 (2013) 6812-6819; (c) M. Khandelwal, R.J. Wehmschulte, Angew. Chem. Int. Ed. 51 (2012) 7323-7326; (d) R. RamírezContreras, N. Bhuvanesh, J. Zhou, O.V. Ozerov, Angew. Chem. Int. Ed. 52 (2013) 10313-10315;

(e) C. Douvris, C.M. Nagaraja, C.-H. Chen, B.M. Foxman, O.V. Ozerov, J. Am. Chem. Soc. 132 (2010) 4946-4953; (f) C. Douvris, O.V. Ozerov, Science 321 (2008) 1188-1190.

[10.] V. Lavallo, J.H. Wright, F.S. Tham, S. Quinlivan, Angew. Chem. Int. Ed. 52 (2013) 3172-3176.

[11.] A. El-Hellani, C.E. Kefalidis, F.S. Tham, L. Maron, V. Lavallo, Organometallics 32 (2013) 68876890.

[12.] For examples of other ligands with carba-closo-dodecaborate substituents, see: (a) M.J. Asay, S.P. Fisher, S.E. Lee, F.S. Tham, D. Borchardt, V. Lavallo, Chem. Commun. 51 (2015) 5359-5362; (b) A. El-Hellani, V. Lavallo, Angew. Chem. Int. Ed. 53 (2014) 4489-4493; (c) A. Himmelspach, M. Finze, S. Raub, Angew. Chem. Int. Ed. 50 (2011) 2628-2631.

[13.] R. Núñez, C. Viñas, F. Teixidor, R. Sillanpää, R. Kivekäs, J. Organomet. Chem. 592 (1999) 22-28.

[14.] N. Fey, M.F. Haddow, R. Mistry, N.C. Norman, A.G. Orpen, T.J. Reynolds, P.G. Pringle, Organometallics 31 (2012) 2907-2913.

[15.] R.H. Crabtree, The Organometallic Chemistry of the Transition Metals, fifth ed., vol. 5, John Wiley \& Sons Ltd., New Jersey, 2009. 


\section{TOC Artwork}
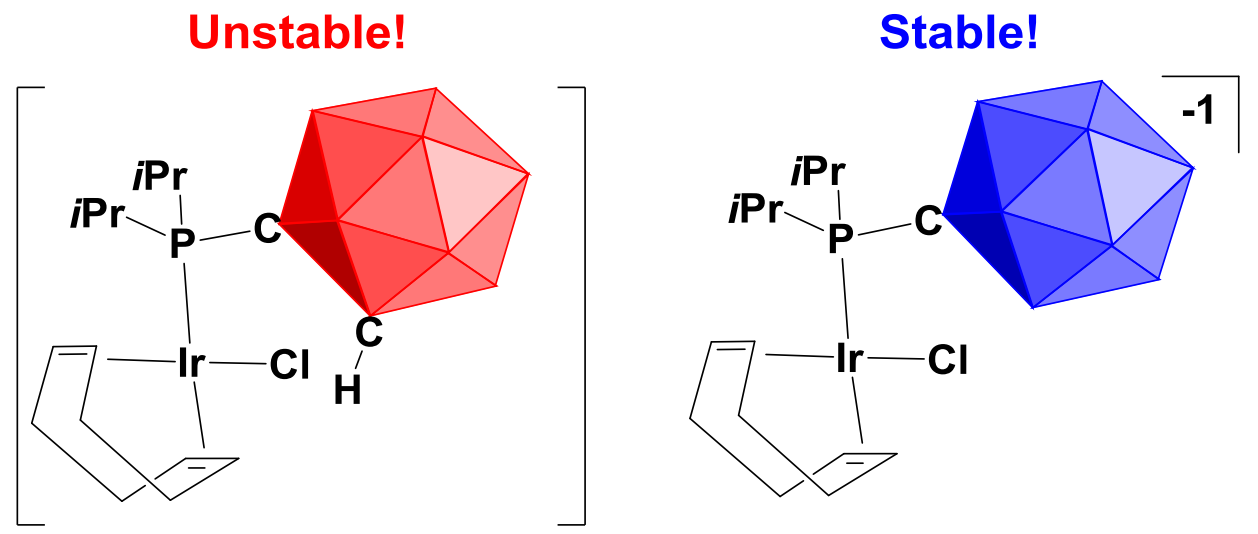

\section{B-H cyclometalation}

The neutral $\operatorname{Ir}(\mathrm{I})$ complex containing the $o$-carboranyl phosphine ligand is not isolable and undergoes spontaneous B-H cyclometalation to afford an Ir(III) hydride. In contrast, the anionic $\operatorname{Ir}(\mathrm{I})$ complex supported by a phosphine with a carba-closo-dodecaborate ligand R-group is stable towards B-H activation. 


\section{$\underline{\text { X-Ray Structure Data, Complex 6" }}$}

A colorless prism fragment $\left(0.173 \times 0.102 \times 0.077 \mathrm{~mm}^{3}\right)$ was used for the single crystal $\mathrm{x}$-ray diffraction study of $\left[\mathrm{C}_{2} \mathrm{H}_{10} \mathrm{~B}_{10}\right]\left[\mathrm{C}_{3} \mathrm{H}_{7}\right]_{2} \mathrm{PC}_{8} \mathrm{H}_{12} \mathrm{ClIrH}$ (sample vL67_0m). The crystal was coated with paratone oil and mounted on to a cryo-loop glass fiber. X-ray intensity data were collected at $100(2) \mathrm{K}$ on a Bruker $\mathrm{APEX} 2^{3}$ platform-CCD x-ray diffractometer system (fine focus Moradiation, $\lambda=0.71073 \AA, 50 \mathrm{KV} / 35 \mathrm{~mA}$ power). The CCD detector was placed at a distance of $5.0800 \mathrm{~cm}$ from the crystal.

A total of 3600 frames were collected for a sphere of reflections (with scan width of $0.3^{\circ}$ in $\omega$ and $\phi$, starting $\omega$ and $2 \theta$ angles of $-30^{\circ}$, and $\phi$ angles of $0^{\circ}, 90^{\circ}, 120^{\circ}, 180^{\circ}, 240^{\circ}$, and $270^{\circ}$ for every 600 frames, $10 \mathrm{sec} /$ frame exposure time). The frames were integrated using the Bruker SAINT software package ${ }^{4}$ and using a narrow-frame integration algorithm. Based on a monoclinic crystal system, the integrated frames yielded a total of 50645 reflections at a maximum $2 \theta$ angle of $59.148^{\circ}(0.72 \AA$ resolution), of which 6585 were independent reflections $\left(\mathrm{R}_{\text {int }}=0.0330, \mathrm{R}_{\mathrm{sig}}=0.0183\right.$, redundancy $=7.7$, completeness $\left.=99.9 \%\right)$ and $6018(91.4 \%)$ reflections were greater than $2 \sigma(\mathrm{I})$. The unit cell parameters were, $\mathbf{a}=13.5940(5) \AA, \mathbf{b}=$ 10.8808(4) $\AA, \mathbf{c}=17.2450(6) \AA, \boldsymbol{\beta}=112.7908(5)^{\mathrm{o}}, \mathrm{V}=2351.62(15) \AA^{3}, \mathrm{Z}=4$, calculated density $D_{c}=1.684 \mathrm{~g} / \mathrm{cm}^{3}$. Absorption corrections were applied (absorption coefficient $\mu=5.862 \mathrm{~mm}^{-1}$; $\mathrm{min} / \mathrm{max}$ transmission $=0.430 / 0.661)$ to the raw intensity data using the SADABS program ${ }^{5}$.

The Bruker SHELXTL software package $^{6}$ was used for phase determination and structure refinement. The distribution of intensities $\left(E^{2}-1=0.862\right)$ and systematic absent reflections indicated one possible space group, P2(1)/n. The space group P2(1)/n (\#14) was later determined to be correct. Direct methods of phase determination followed by two Fourier cycles of refinement led to an electron density map from which most of the non-hydrogen atoms were identified in the asymmetric unit of the unit cell. With subsequent isotropic refinement, all of the non-hydrogen atoms were identified. There was one molecule of $\left[\mathrm{C}_{2} \mathrm{H}_{10} \mathrm{~B}_{10}\right]\left[\mathrm{C}_{3} \mathrm{H}_{7}\right]_{2} \mathrm{PC}_{8} \mathrm{H}_{12} \mathrm{ClIrH}$ present in the asymmetric unit of the unit cell.

Atomic coordinates, isotropic and anisotropic displacement parameters of all the non-hydrogen atoms were refined by means of a full matrix least-squares procedure on $\mathrm{F}^{2}$. The $\mathrm{H}$-atoms were 
included in the refinement in calculated positions riding on the atoms to which they were attached, except the hydride atom and four of the $\mathrm{H}$-atoms of the $\mathrm{C}_{8} \mathrm{H}_{12}$-group were refined unrestrained. The refinement converged at R1 $=0.0164$, $w R 2=0.0349$, with intensity $I>2 \sigma$ (I). The largest peak/hole in the final difference map was $0.927 /-0.782 \mathrm{e} / \AA^{3}$.

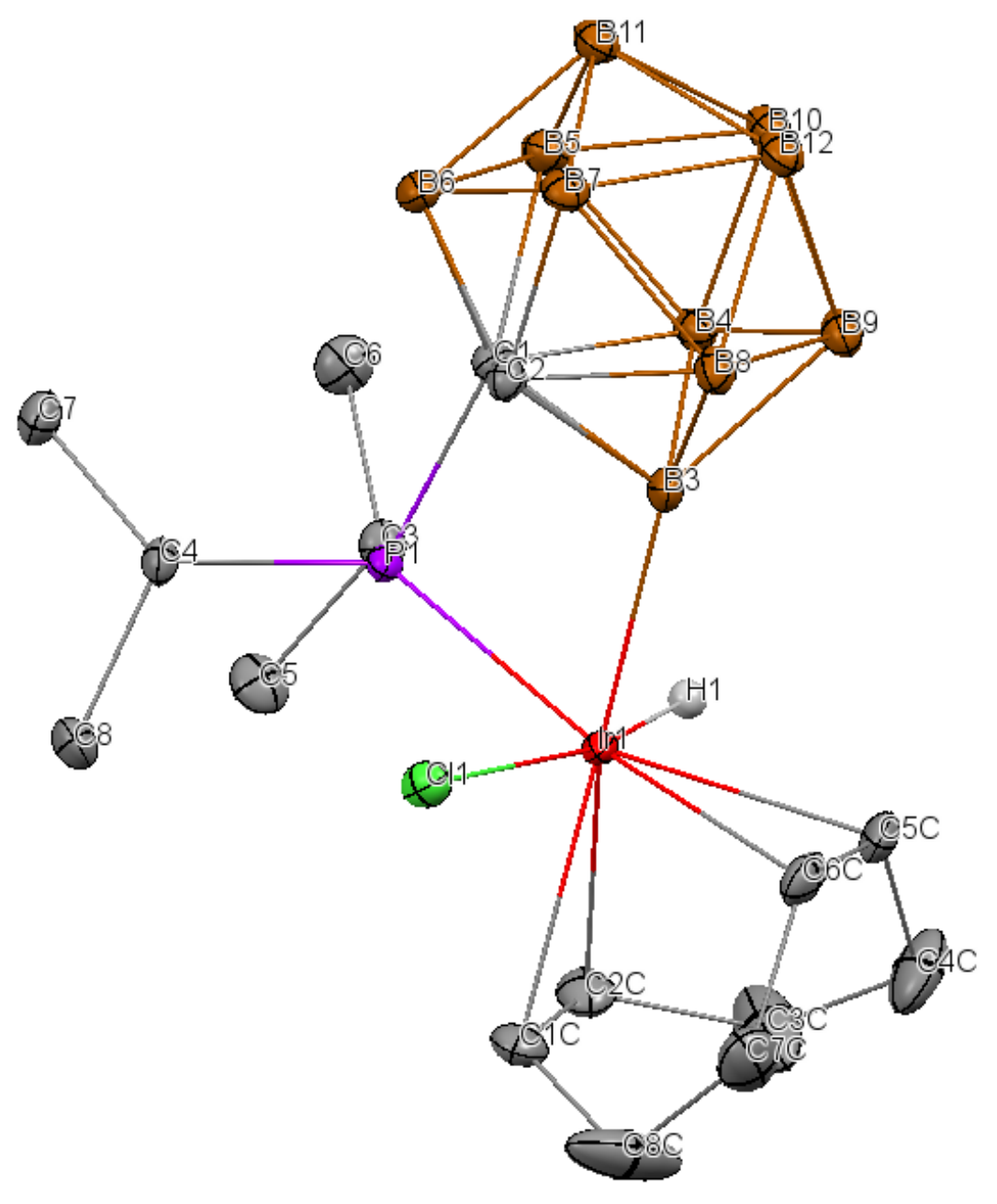

Full crystallographic data for 6" can be found at the Cambridge crystallographic database under reference \# CCDC 1057296 
Table 1. Crystal data and structure refinement for vL67_0m.

Identification code

Empirical formula

Formula weight

Temperature

Wavelength

Crystal system

Space group

Unit cell dimensions

Volume

$\mathrm{Z}$

Density (calculated)

Absorption coefficient

$\mathrm{F}(000)$

Crystal size

Theta range for data collection

Index ranges

Reflections collected

Independent reflections

Completeness to theta $=25.242^{\circ}$

Absorption correction

Refinement method

Data / restraints / parameters

Goodness-of-fit on $\mathrm{F}^{2}$

Final $\mathrm{R}$ indices [I $>2 \operatorname{sigma}(\mathrm{I})]$

$\mathrm{R}$ indices (all data)

Extinction coefficient

Largest diff. peak and hole
vL67_0m

C16 H37 B10 Cl Ir P

596.17

100(2) K

$0.71073 \AA$

Monoclinic

P 21/n (\#14)

$\mathrm{a}=13.5940(5) \AA \quad \alpha=90^{\circ}$.

$\mathrm{b}=10.8808(4) \AA$

$\beta=112.7908(5)^{\circ}$.

$\mathrm{c}=17.2450(6) \AA$

$\gamma=90^{\circ}$.
4

$1.684 \mathrm{Mg} / \mathrm{m}^{3}$

$5.862 \mathrm{~mm}^{-1}$

1168

$0.173 \times 0.102 \times 0.077 \mathrm{~mm}^{3}$

2.268 to $29.574^{\circ}$.

$-18<=\mathrm{h}<=18,-15<=\mathrm{k}<=15,-23<=\mathrm{l}<=23$

50645

$6585[\mathrm{R}(\mathrm{int})=0.0330]$

$100.0 \%$

Semi-empirical from equivalents

Full-matrix least-squares on $\mathrm{F}^{2}$

6585 / 0 / 288

1.051

$\mathrm{R} 1=0.0164, \mathrm{wR} 2=0.0349$

$\mathrm{R} 1=0.0198, \mathrm{wR} 2=0.0362$

$\mathrm{n} / \mathrm{a}$

0.927 and -0.782 e. $\AA^{-3}$ 
Table 2. Atomic coordinates ( $\left.\mathrm{x} 10^{4}\right)$ and equivalent isotropic displacement parameters $\left(\AA^{2} \mathrm{x}\right.$ $10^{3}$ ) for vL67_0m. U(eq) is defined as one third of the trace of the orthogonalized Uij tensor.

\begin{tabular}{|c|c|c|c|c|}
\hline & $\mathrm{x}$ & $\mathrm{y}$ & $\mathrm{z}$ & $\mathrm{U}(\mathrm{eq})$ \\
\hline $\operatorname{Ir}(1)$ & $2448(1)$ & $1910(1)$ & $70(1)$ & $8(1)$ \\
\hline $\mathrm{Cl}(1)$ & $595(1)$ & $1723(1)$ & $26(1)$ & $15(1)$ \\
\hline$C(1 C)$ & $2471(2)$ & $3511(2)$ & $999(1)$ & $18(1)$ \\
\hline$C(2 C)$ & $3496(2)$ & $3323(2)$ & $1077(1)$ & $20(1)$ \\
\hline$C(3 C)$ & 4104(3) & $4082(3)$ & $689(2)$ & $49(1)$ \\
\hline$C(4 C)$ & $3604(2)$ & $4243(2)$ & $-254(2)$ & $32(1)$ \\
\hline$C(5 C)$ & $2734(2)$ & $3345(2)$ & $-751(1)$ & $18(1)$ \\
\hline$C(6 C)$ & $1686(2)$ & $3438(2)$ & $-822(1)$ & $18(1)$ \\
\hline$C(7 C)$ & $1252(2)$ & $4421(2)$ & $-428(2)$ & $30(1)$ \\
\hline $\mathrm{C}(8 \mathrm{C})$ & 1803(3) & 4578(3) & $506(2)$ & $47(1)$ \\
\hline $\mathrm{C}(1)$ & $2513(1)$ & $-842(2)$ & $-242(1)$ & $10(1)$ \\
\hline $\mathrm{C}(2)$ & $1252(2)$ & $-602(2)$ & $-816(1)$ & $10(1)$ \\
\hline $\mathrm{B}(3)$ & 2159(2) & $529(2)$ & $-837(1)$ & $10(1)$ \\
\hline $\mathrm{B}(4)$ & $3266(2)$ & $-454(2)$ & $-790(1)$ & $12(1)$ \\
\hline $\mathrm{B}(5)$ & 2981(2) & $-2025(2)$ & $-666(1)$ & $14(1)$ \\
\hline $\mathrm{B}(6)$ & $1689(2)$ & $-2091(2)$ & $-645(1)$ & $12(1)$ \\
\hline $\mathrm{B}(7)$ & $769(2)$ & $-1561(2)$ & $-1643(1)$ & $13(1)$ \\
\hline $\mathrm{B}(8)$ & $1060(2)$ & $24(2)$ & $-1760(1)$ & $13(1)$ \\
\hline $\mathrm{B}(9)$ & $2354(2)$ & $98(2)$ & $-1776(1)$ & $14(1)$ \\
\hline $\mathrm{B}(10)$ & $2864(2)$ & $-1446(2)$ & $-1665(1)$ & $14(1)$ \\
\hline $\mathrm{B}(11)$ & 1876(2) & $-2471(2)$ & $-1579(1)$ & $14(1)$ \\
\hline $\mathrm{B}(12)$ & $1494(2)$ & $-1159(2)$ & $-2269(1)$ & $14(1)$ \\
\hline $\mathrm{P}(1)$ & 2894(1) & 39(1) & $765(1)$ & $9(1)$ \\
\hline $\mathrm{C}(3)$ & $4340(2)$ & $-187(2)$ & $1374(1)$ & $13(1)$ \\
\hline $\mathrm{C}(4)$ & 2098(2) & $-633(2)$ & $1315(1)$ & $12(1)$ \\
\hline$C(5)$ & $4650(2)$ & $264(2)$ & $2285(1)$ & 19(1) \\
\hline$C(6)$ & $4796(2)$ & $-1472(2)$ & $1367(1)$ & $18(1)$ \\
\hline $\mathrm{C}(7)$ & $2424(2)$ & $-1939(2)$ & $1657(1)$ & $18(1)$ \\
\hline $\mathrm{C}(8)$ & 2031(2) & $237(2)$ & 1994(1) & $17(1)$ \\
\hline
\end{tabular}


Table 3. Bond lengths $[\AA]$ and angles $\left[{ }^{\circ}\right]$ for vL67_0m.

\begin{tabular}{|c|c|c|c|}
\hline $\operatorname{Ir}(1)-B(3)$ & $2.094(2)$ & $\mathrm{C}(2)-\mathrm{B}(7)$ & $1.683(3)$ \\
\hline $\operatorname{Ir}(1)-C(6 C)$ & $2.230(2)$ & $\mathrm{C}(2)-\mathrm{B}(8)$ & $1.689(3)$ \\
\hline $\operatorname{Ir}(1)-C(5 C)$ & $2.242(2)$ & $\mathrm{C}(2)-\mathrm{B}(6)$ & $1.712(3)$ \\
\hline $\operatorname{Ir}(1)-\mathrm{P}(1)$ & $2.3203(5)$ & $\mathrm{C}(2)-\mathrm{B}(3)$ & $1.752(3)$ \\
\hline $\operatorname{Ir}(1)-C(2 C)$ & $2.344(2)$ & $\mathrm{C}(2)-\mathrm{H}(2)$ & $0.93(2)$ \\
\hline $\operatorname{Ir}(1)-C(1 C)$ & $2.359(2)$ & $\mathrm{B}(3)-\mathrm{B}(8)$ & $1.796(3)$ \\
\hline $\operatorname{Ir}(1)-\mathrm{Cl}(1)$ & $2.4976(5)$ & $\mathrm{B}(3)-\mathrm{B}(9)$ & $1.801(3)$ \\
\hline $\operatorname{Ir}(1)-\mathrm{H}(1)$ & $1.45(2)$ & $\mathrm{B}(3)-\mathrm{B}(4)$ & $1.822(3)$ \\
\hline$C(1 C)-C(2 C)$ & $1.362(3)$ & $\mathrm{B}(4)-\mathrm{B}(10)$ & $1.761(3)$ \\
\hline$C(1 C)-C(8 C)$ & $1.515(3)$ & $\mathrm{B}(4)-\mathrm{B}(9)$ & $1.775(3)$ \\
\hline $\mathrm{C}(1 \mathrm{C})-\mathrm{H}(1 \mathrm{C})$ & $0.93(3)$ & $\mathrm{B}(4)-\mathrm{B}(5)$ & $1.784(3)$ \\
\hline $\mathrm{C}(2 \mathrm{C})-\mathrm{C}(3 \mathrm{C})$ & $1.498(3)$ & $\mathrm{B}(4)-\mathrm{H}(4)$ & 1.1200 \\
\hline $\mathrm{C}(2 \mathrm{C})-\mathrm{H}(2 \mathrm{C})$ & $0.98(3)$ & $\mathrm{B}(5)-\mathrm{B}(11)$ & $1.771(3)$ \\
\hline $\mathrm{C}(3 \mathrm{C})-\mathrm{C}(4 \mathrm{C})$ & $1.510(4)$ & $\mathrm{B}(5)-\mathrm{B}(6)$ & $1.771(3)$ \\
\hline $\mathrm{C}(3 \mathrm{C})-\mathrm{H}(3 \mathrm{~B})$ & 0.9900 & $\mathrm{~B}(5)-\mathrm{B}(10)$ & $1.783(3)$ \\
\hline $\mathrm{C}(3 \mathrm{C})-\mathrm{H}(3 \mathrm{C})$ & 0.9900 & $\mathrm{~B}(5)-\mathrm{H}(5)$ & 1.1200 \\
\hline $\mathrm{C}(4 \mathrm{C})-\mathrm{C}(5 \mathrm{C})$ & $1.517(3)$ & $\mathrm{B}(6)-\mathrm{B}(11)$ & $1.773(3)$ \\
\hline $\mathrm{C}(4 \mathrm{C})-\mathrm{H}(4 \mathrm{~B})$ & 0.9900 & $\mathrm{~B}(6)-\mathrm{B}(7)$ & $1.786(3)$ \\
\hline $\mathrm{C}(4 \mathrm{C})-\mathrm{H}(4 \mathrm{C})$ & 0.9900 & $\mathrm{~B}(6)-\mathrm{H}(6)$ & 1.1200 \\
\hline$C(5 C)-C(6 C)$ & $1.384(3)$ & $\mathrm{B}(7)-\mathrm{B}(11)$ & $1.769(3)$ \\
\hline $\mathrm{C}(5 \mathrm{C})-\mathrm{H}(5 \mathrm{D})$ & $1.00(3)$ & $\mathrm{B}(7)-\mathrm{B}(12)$ & $1.775(3)$ \\
\hline$C(6 C)-C(7 C)$ & $1.506(3)$ & $\mathrm{B}(7)-\mathrm{B}(8)$ & $1.798(3)$ \\
\hline $\mathrm{C}(6 \mathrm{C})-\mathrm{H}(6 \mathrm{D})$ & $0.96(3)$ & $\mathrm{B}(7)-\mathrm{H}(7)$ & 1.1200 \\
\hline$C(7 C)-C(8 C)$ & $1.499(4)$ & $\mathrm{B}(8)-\mathrm{B}(9)$ & $1.773(3)$ \\
\hline $\mathrm{C}(7 \mathrm{C})-\mathrm{H}(7 \mathrm{D})$ & 0.9900 & $\mathrm{~B}(8)-\mathrm{B}(12)$ & $1.782(3)$ \\
\hline $\mathrm{C}(7 \mathrm{C})-\mathrm{H}(7 \mathrm{E})$ & 0.9900 & $\mathrm{~B}(8)-\mathrm{H}(8)$ & 1.1200 \\
\hline $\mathrm{C}(8 \mathrm{C})-\mathrm{H}(8 \mathrm{D})$ & 0.9900 & $\mathrm{~B}(9)-\mathrm{B}(12)$ & $1.787(3)$ \\
\hline $\mathrm{C}(8 \mathrm{C})-\mathrm{H}(8 \mathrm{E})$ & 0.9900 & $\mathrm{~B}(9)-\mathrm{B}(10)$ & $1.799(3)$ \\
\hline $\mathrm{C}(1)-\mathrm{C}(2)$ & $1.634(3)$ & $\mathrm{B}(9)-\mathrm{H}(9)$ & 1.1200 \\
\hline $\mathrm{C}(1)-\mathrm{B}(4)$ & $1.694(3)$ & $\mathrm{B}(10)-\mathrm{B}(12)$ & $1.774(3)$ \\
\hline $\mathrm{C}(1)-\mathrm{B}(5)$ & $1.720(3)$ & $\mathrm{B}(10)-\mathrm{B}(11)$ & $1.795(3)$ \\
\hline$C(1)-B(6)$ & $1.727(3)$ & $\mathrm{B}(10)-\mathrm{H}(10)$ & 1.1200 \\
\hline $\mathrm{C}(1)-\mathrm{B}(3)$ & $1.768(3)$ & $\mathrm{B}(11)-\mathrm{B}(12)$ & $1.801(3)$ \\
\hline $\mathrm{C}(1)-\mathrm{P}(1)$ & $1.8728(19)$ & $\mathrm{B}(11)-\mathrm{H}(11)$ & 1.1200 \\
\hline
\end{tabular}




\begin{tabular}{|c|c|c|c|}
\hline $\mathrm{B}(12)-\mathrm{H}(12)$ & 1.1200 & $C(2 C)-\operatorname{Ir}(1)-C(1 C)$ & $33.66(8)$ \\
\hline $\mathrm{P}(1)-\mathrm{C}(4)$ & $1.8441(18)$ & $\mathrm{B}(3)-\operatorname{Ir}(1)-\mathrm{Cl}(1)$ & $91.36(6)$ \\
\hline $\mathrm{P}(1)-\mathrm{C}(3)$ & $1.851(2)$ & $\mathrm{C}(6 \mathrm{C})-\operatorname{Ir}(1)-\mathrm{Cl}(1)$ & $81.17(6)$ \\
\hline$C(3)-C(6)$ & $1.532(3)$ & $\mathrm{C}(5 \mathrm{C})-\operatorname{Ir}(1)-\mathrm{Cl}(1)$ & $117.17(6)$ \\
\hline$C(3)-C(5)$ & $1.539(3)$ & $\mathrm{P}(1)-\operatorname{Ir}(1)-\mathrm{Cl}(1)$ & $90.218(16)$ \\
\hline $\mathrm{C}(3)-\mathrm{H}(3 \mathrm{~A})$ & 1.0000 & $\mathrm{C}(2 \mathrm{C})-\operatorname{Ir}(1)-\mathrm{Cl}(1)$ & $113.12(6)$ \\
\hline$C(4)-C(8)$ & $1.536(3)$ & $\mathrm{C}(1 \mathrm{C})-\operatorname{Ir}(1)-\mathrm{Cl}(1)$ & $80.18(6)$ \\
\hline$C(4)-C(7)$ & $1.537(3)$ & $\mathrm{B}(3)-\operatorname{Ir}(1)-\mathrm{H}(1)$ & $82.0(9)$ \\
\hline $\mathrm{C}(4)-\mathrm{H}(4 \mathrm{~A})$ & 1.0000 & $\mathrm{C}(6 \mathrm{C})-\operatorname{Ir}(1)-\mathrm{H}(1)$ & $105.3(9)$ \\
\hline $\mathrm{C}(5)-\mathrm{H}(5 \mathrm{~A})$ & 0.9800 & $\mathrm{C}(5 \mathrm{C})-\operatorname{Ir}(1)-\mathrm{H}(1)$ & $69.3(9)$ \\
\hline $\mathrm{C}(5)-\mathrm{H}(5 \mathrm{~B})$ & 0.9800 & $\mathrm{P}(1)-\operatorname{Ir}(1)-\mathrm{H}(1)$ & $82.2(9)$ \\
\hline $\mathrm{C}(5)-\mathrm{H}(5 \mathrm{C})$ & 0.9800 & $\mathrm{C}(2 \mathrm{C})-\operatorname{Ir}(1)-\mathrm{H}(1)$ & $73.4(9)$ \\
\hline $\mathrm{C}(6)-\mathrm{H}(6 \mathrm{~A})$ & 0.9800 & $\mathrm{C}(1 \mathrm{C})-\operatorname{Ir}(1)-\mathrm{H}(1)$ & $106.8(9)$ \\
\hline $\mathrm{C}(6)-\mathrm{H}(6 \mathrm{~B})$ & 0.9800 & $\mathrm{Cl}(1)-\operatorname{Ir}(1)-\mathrm{H}(1)$ & 171.1(9) \\
\hline $\mathrm{C}(6)-\mathrm{H}(6 \mathrm{C})$ & 0.9800 & $C(2 C)-C(1 C)-C(8 C)$ & $122.5(2)$ \\
\hline $\mathrm{C}(7)-\mathrm{H}(7 \mathrm{~A})$ & 0.9800 & $C(2 C)-C(1 C)-\operatorname{Ir}(1)$ & $72.55(12)$ \\
\hline $\mathrm{C}(7)-\mathrm{H}(7 \mathrm{~B})$ & 0.9800 & $C(8 C)-C(1 C)-\operatorname{Ir}(1)$ & $109.69(15)$ \\
\hline $\mathrm{C}(7)-\mathrm{H}(7 \mathrm{C})$ & 0.9800 & $\mathrm{C}(2 \mathrm{C})-\mathrm{C}(1 \mathrm{C})-\mathrm{H}(1 \mathrm{C})$ & $118.0(17)$ \\
\hline $\mathrm{C}(8)-\mathrm{H}(8 \mathrm{~A})$ & 0.9800 & $\mathrm{C}(8 \mathrm{C})-\mathrm{C}(1 \mathrm{C})-\mathrm{H}(1 \mathrm{C})$ & 117.1(17) \\
\hline $\mathrm{C}(8)-\mathrm{H}(8 \mathrm{~B})$ & 0.9800 & $\operatorname{Ir}(1)-C(1 C)-H(1 C)$ & $102.5(16)$ \\
\hline \multirow[t]{2}{*}{$\mathrm{C}(8)-\mathrm{H}(8 \mathrm{C})$} & 0.9800 & $\mathrm{C}(1 \mathrm{C})-\mathrm{C}(2 \mathrm{C})-\mathrm{C}(3 \mathrm{C})$ & $126.4(3)$ \\
\hline & & $C(1 C)-C(2 C)-\operatorname{Ir}(1)$ & $73.79(13)$ \\
\hline $\mathrm{B}(3)-\operatorname{Ir}(1)-\mathrm{C}(6 \mathrm{C})$ & $96.69(8)$ & $C(3 C)-C(2 C)-\operatorname{Ir}(1)$ & $108.25(15)$ \\
\hline $\mathrm{B}(3)-\operatorname{Ir}(1)-\mathrm{C}(5 \mathrm{C})$ & $93.20(8)$ & $\mathrm{C}(1 \mathrm{C})-\mathrm{C}(2 \mathrm{C})-\mathrm{H}(2 \mathrm{C})$ & $117.4(16)$ \\
\hline$C(6 C)-\operatorname{Ir}(1)-C(5 C)$ & $36.07(8)$ & $\mathrm{C}(3 \mathrm{C})-\mathrm{C}(2 \mathrm{C})-\mathrm{H}(2 \mathrm{C})$ & $114.2(16)$ \\
\hline $\mathrm{B}(3)-\operatorname{Ir}(1)-\mathrm{P}(1)$ & $72.09(6)$ & $\operatorname{Ir}(1)-\mathrm{C}(2 \mathrm{C})-\mathrm{H}(2 \mathrm{C})$ & $102.6(16)$ \\
\hline $\mathrm{C}(6 \mathrm{C})-\operatorname{Ir}(1)-\mathrm{P}(1)$ & $165.78(6)$ & $\mathrm{C}(2 \mathrm{C})-\mathrm{C}(3 \mathrm{C})-\mathrm{C}(4 \mathrm{C})$ & $116.7(2)$ \\
\hline $\mathrm{C}(5 \mathrm{C})-\operatorname{Ir}(1)-\mathrm{P}(1)$ & $149.65(6)$ & $\mathrm{C}(2 \mathrm{C})-\mathrm{C}(3 \mathrm{C})-\mathrm{H}(3 \mathrm{~B})$ & 108.1 \\
\hline $\mathrm{B}(3)-\operatorname{Ir}(1)-\mathrm{C}(2 \mathrm{C})$ & $155.32(8)$ & $\mathrm{C}(4 \mathrm{C})-\mathrm{C}(3 \mathrm{C})-\mathrm{H}(3 \mathrm{~B})$ & 108.1 \\
\hline$C(6 C)-\operatorname{Ir}(1)-C(2 C)$ & $90.48(9)$ & $\mathrm{C}(2 \mathrm{C})-\mathrm{C}(3 \mathrm{C})-\mathrm{H}(3 \mathrm{C})$ & 108.1 \\
\hline$C(5 C)-\operatorname{Ir}(1)-C(2 C)$ & $78.83(8)$ & $\mathrm{C}(4 \mathrm{C})-\mathrm{C}(3 \mathrm{C})-\mathrm{H}(3 \mathrm{C})$ & 108.1 \\
\hline$P(1)-\operatorname{Ir}(1)-C(2 C)$ & $103.35(6)$ & $\mathrm{H}(3 \mathrm{~B})-\mathrm{C}(3 \mathrm{C})-\mathrm{H}(3 \mathrm{C})$ & 107.3 \\
\hline $\mathrm{B}(3)-\operatorname{Ir}(1)-\mathrm{C}(1 \mathrm{C})$ & $170.73(8)$ & $\mathrm{C}(3 \mathrm{C})-\mathrm{C}(4 \mathrm{C})-\mathrm{C}(5 \mathrm{C})$ & $117.4(2)$ \\
\hline$C(6 C)-\operatorname{Ir}(1)-C(1 C)$ & $78.37(8)$ & $\mathrm{C}(3 \mathrm{C})-\mathrm{C}(4 \mathrm{C})-\mathrm{H}(4 \mathrm{~B})$ & 107.9 \\
\hline$C(5 C)-\operatorname{Ir}(1)-C(1 C)$ & $87.37(8)$ & $\mathrm{C}(5 \mathrm{C})-\mathrm{C}(4 \mathrm{C})-\mathrm{H}(4 \mathrm{~B})$ & 107.9 \\
\hline$P(1)-\operatorname{Ir}(1)-C(1 C)$ & $111.44(6)$ & $\mathrm{C}(3 \mathrm{C})-\mathrm{C}(4 \mathrm{C})-\mathrm{H}(4 \mathrm{C})$ & 107.9 \\
\hline
\end{tabular}




\begin{tabular}{|c|c|c|c|}
\hline $\mathrm{C}(5 \mathrm{C})-\mathrm{C}(4 \mathrm{C})-\mathrm{H}(4 \mathrm{C})$ & 107.9 & $\mathrm{C}(2)-\mathrm{C}(1)-\mathrm{P}(1)$ & $109.18(12)$ \\
\hline $\mathrm{H}(4 \mathrm{~B})-\mathrm{C}(4 \mathrm{C})-\mathrm{H}(4 \mathrm{C})$ & 107.2 & $\mathrm{~B}(4)-\mathrm{C}(1)-\mathrm{P}(1)$ & $112.33(13)$ \\
\hline$C(6 C)-C(5 C)-C(4 C)$ & $123.6(2)$ & $\mathrm{B}(5)-\mathrm{C}(1)-\mathrm{P}(1)$ & $139.66(14)$ \\
\hline$C(6 C)-C(5 C)-\operatorname{Ir}(1)$ & $71.49(12)$ & $\mathrm{B}(6)-\mathrm{C}(1)-\mathrm{P}(1)$ & $132.85(13)$ \\
\hline$C(4 C)-C(5 C)-\operatorname{Ir}(1)$ & $112.35(15)$ & $\mathrm{B}(3)-\mathrm{C}(1)-\mathrm{P}(1)$ & $91.26(11)$ \\
\hline $\mathrm{C}(6 \mathrm{C})-\mathrm{C}(5 \mathrm{C})-\mathrm{H}(5 \mathrm{D})$ & $117.3(16)$ & $\mathrm{C}(1)-\mathrm{C}(2)-\mathrm{B}(7)$ & $112.80(15)$ \\
\hline $\mathrm{C}(4 \mathrm{C})-\mathrm{C}(5 \mathrm{C})-\mathrm{H}(5 \mathrm{D})$ & $115.3(16)$ & $\mathrm{C}(1)-\mathrm{C}(2)-\mathrm{B}(8)$ & $112.48(14)$ \\
\hline $\operatorname{Ir}(1)-C(5 C)-H(5 D)$ & $105.2(15)$ & $\mathrm{B}(7)-\mathrm{C}(2)-\mathrm{B}(8)$ & $64.46(13)$ \\
\hline $\mathrm{C}(5 \mathrm{C})-\mathrm{C}(6 \mathrm{C})-\mathrm{C}(7 \mathrm{C})$ & $125.5(2)$ & $\mathrm{C}(1)-\mathrm{C}(2)-\mathrm{B}(6)$ & $62.09(12)$ \\
\hline $\mathrm{C}(5 \mathrm{C})-\mathrm{C}(6 \mathrm{C})-\operatorname{Ir}(1)$ & $72.44(12)$ & $\mathrm{B}(7)-\mathrm{C}(2)-\mathrm{B}(6)$ & $63.48(13)$ \\
\hline$C(7 C)-C(6 C)-\operatorname{Ir}(1)$ & 112.11(14) & $\mathrm{B}(8)-\mathrm{C}(2)-\mathrm{B}(6)$ & $117.43(15)$ \\
\hline $\mathrm{C}(5 \mathrm{C})-\mathrm{C}(6 \mathrm{C})-\mathrm{H}(6 \mathrm{D})$ & $117.5(16)$ & $\mathrm{C}(1)-\mathrm{C}(2)-\mathrm{B}(3)$ & $62.84(12)$ \\
\hline $\mathrm{C}(7 \mathrm{C})-\mathrm{C}(6 \mathrm{C})-\mathrm{H}(6 \mathrm{D})$ & $113.7(16)$ & $\mathrm{B}(7)-\mathrm{C}(2)-\mathrm{B}(3)$ & $117.68(14)$ \\
\hline $\operatorname{Ir}(1)-C(6 C)-H(6 D)$ & $103.9(16)$ & $\mathrm{B}(8)-\mathrm{C}(2)-\mathrm{B}(3)$ & $62.87(12)$ \\
\hline$C(8 C)-C(7 C)-C(6 C)$ & $116.4(2)$ & $\mathrm{B}(6)-\mathrm{C}(2)-\mathrm{B}(3)$ & $117.90(15)$ \\
\hline $\mathrm{C}(8 \mathrm{C})-\mathrm{C}(7 \mathrm{C})-\mathrm{H}(7 \mathrm{D})$ & 108.2 & $\mathrm{C}(1)-\mathrm{C}(2)-\mathrm{H}(2)$ & $116.0(14)$ \\
\hline $\mathrm{C}(6 \mathrm{C})-\mathrm{C}(7 \mathrm{C})-\mathrm{H}(7 \mathrm{D})$ & 108.2 & $\mathrm{~B}(7)-\mathrm{C}(2)-\mathrm{H}(2)$ & $121.2(14)$ \\
\hline $\mathrm{C}(8 \mathrm{C})-\mathrm{C}(7 \mathrm{C})-\mathrm{H}(7 \mathrm{E})$ & 108.2 & $\mathrm{~B}(8)-\mathrm{C}(2)-\mathrm{H}(2)$ & $119.9(14)$ \\
\hline $\mathrm{C}(6 \mathrm{C})-\mathrm{C}(7 \mathrm{C})-\mathrm{H}(7 \mathrm{E})$ & 108.2 & $\mathrm{~B}(6)-\mathrm{C}(2)-\mathrm{H}(2)$ & $115.5(14)$ \\
\hline$H(7 D)-C(7 C)-H(7 E)$ & 107.3 & $\mathrm{~B}(3)-\mathrm{C}(2)-\mathrm{H}(2)$ & $112.6(14)$ \\
\hline$C(7 C)-C(8 C)-C(1 C)$ & $117.2(2)$ & $\mathrm{C}(2)-\mathrm{B}(3)-\mathrm{C}(1)$ & $55.31(11)$ \\
\hline $\mathrm{C}(7 \mathrm{C})-\mathrm{C}(8 \mathrm{C})-\mathrm{H}(8 \mathrm{D})$ & 108.0 & $\mathrm{C}(2)-\mathrm{B}(3)-\mathrm{B}(8)$ & $56.85(11)$ \\
\hline $\mathrm{C}(1 \mathrm{C})-\mathrm{C}(8 \mathrm{C})-\mathrm{H}(8 \mathrm{D})$ & 108.0 & $\mathrm{C}(1)-\mathrm{B}(3)-\mathrm{B}(8)$ & $101.65(14)$ \\
\hline $\mathrm{C}(7 \mathrm{C})-\mathrm{C}(8 \mathrm{C})-\mathrm{H}(8 \mathrm{E})$ & 108.0 & $\mathrm{C}(2)-\mathrm{B}(3)-\mathrm{B}(9)$ & $100.74(14)$ \\
\hline $\mathrm{C}(1 \mathrm{C})-\mathrm{C}(8 \mathrm{C})-\mathrm{H}(8 \mathrm{E})$ & 108.0 & $\mathrm{C}(1)-\mathrm{B}(3)-\mathrm{B}(9)$ & $101.56(14)$ \\
\hline $\mathrm{H}(8 \mathrm{D})-\mathrm{C}(8 \mathrm{C})-\mathrm{H}(8 \mathrm{E})$ & 107.2 & $\mathrm{~B}(8)-\mathrm{B}(3)-\mathrm{B}(9)$ & $59.07(12)$ \\
\hline $\mathrm{C}(2)-\mathrm{C}(1)-\mathrm{B}(4)$ & 109.94(14) & $\mathrm{C}(2)-\mathrm{B}(3)-\mathrm{B}(4)$ & $99.37(14)$ \\
\hline $\mathrm{C}(2)-\mathrm{C}(1)-\mathrm{B}(5)$ & 109.66(15) & $\mathrm{C}(1)-\mathrm{B}(3)-\mathrm{B}(4)$ & $56.29(11)$ \\
\hline $\mathrm{B}(4)-\mathrm{C}(1)-\mathrm{B}(5)$ & $63.02(12)$ & $\mathrm{B}(8)-\mathrm{B}(3)-\mathrm{B}(4)$ & $104.43(15)$ \\
\hline $\mathrm{C}(2)-\mathrm{C}(1)-\mathrm{B}(6)$ & $61.18(12)$ & $\mathrm{B}(9)-\mathrm{B}(3)-\mathrm{B}(4)$ & $58.68(12)$ \\
\hline $\mathrm{B}(4)-\mathrm{C}(1)-\mathrm{B}(6)$ & $114.14(15)$ & $\mathrm{C}(2)-\mathrm{B}(3)-\operatorname{Ir}(1)$ & $114.69(12)$ \\
\hline $\mathrm{B}(5)-\mathrm{C}(1)-\mathrm{B}(6)$ & $61.85(12)$ & $\mathrm{C}(1)-\mathrm{B}(3)-\operatorname{Ir}(1)$ & $103.96(11)$ \\
\hline $\mathrm{C}(2)-\mathrm{C}(1)-\mathrm{B}(3)$ & $61.85(12)$ & $\mathrm{B}(8)-\mathrm{B}(3)-\operatorname{Ir}(1)$ & $137.10(14)$ \\
\hline $\mathrm{B}(4)-\mathrm{C}(1)-\mathrm{B}(3)$ & $63.45(12)$ & $\mathrm{B}(9)-\mathrm{B}(3)-\operatorname{Ir}(1)$ & $143.92(14)$ \\
\hline $\mathrm{B}(5)-\mathrm{C}(1)-\mathrm{B}(3)$ & $116.55(14)$ & $\mathrm{B}(4)-\mathrm{B}(3)-\operatorname{Ir}(1)$ & $118.39(13)$ \\
\hline $\mathrm{B}(6)-\mathrm{C}(1)-\mathrm{B}(3)$ & $116.25(14)$ & $\mathrm{C}(1)-\mathrm{B}(4)-\mathrm{B}(10)$ & $105.73(15)$ \\
\hline
\end{tabular}




\begin{tabular}{|c|c|c|c|}
\hline $\mathrm{C}(1)-\mathrm{B}(4)-\mathrm{B}(9)$ & $105.68(15)$ & $\mathrm{C}(1)-\mathrm{B}(6)-\mathrm{B}(7)$ & $103.69(15)$ \\
\hline $\mathrm{B}(10)-\mathrm{B}(4)-\mathrm{B}(9)$ & $61.15(13)$ & $\mathrm{B}(5)-\mathrm{B}(6)-\mathrm{B}(7)$ & $107.31(15)$ \\
\hline $\mathrm{C}(1)-\mathrm{B}(4)-\mathrm{B}(5)$ & $59.20(12)$ & $\mathrm{B}(11)-\mathrm{B}(6)-\mathrm{B}(7)$ & $59.60(12)$ \\
\hline $\mathrm{B}(10)-\mathrm{B}(4)-\mathrm{B}(5)$ & $60.37(13)$ & $\mathrm{C}(2)-\mathrm{B}(6)-\mathrm{H}(6)$ & 125.7 \\
\hline $\mathrm{B}(9)-\mathrm{B}(4)-\mathrm{B}(5)$ & 109.62(16) & $\mathrm{C}(1)-\mathrm{B}(6)-\mathrm{H}(6)$ & 124.6 \\
\hline $\mathrm{C}(1)-\mathrm{B}(4)-\mathrm{B}(3)$ & $60.26(11)$ & $\mathrm{B}(5)-\mathrm{B}(6)-\mathrm{H}(6)$ & 122.2 \\
\hline $\mathrm{B}(10)-\mathrm{B}(4)-\mathrm{B}(3)$ & 110.18(16) & $\mathrm{B}(11)-\mathrm{B}(6)-\mathrm{H}(6)$ & 123.4 \\
\hline $\mathrm{B}(9)-\mathrm{B}(4)-\mathrm{B}(3)$ & $60.07(12)$ & $\mathrm{B}(7)-\mathrm{B}(6)-\mathrm{H}(6)$ & 122.9 \\
\hline $\mathrm{B}(5)-\mathrm{B}(4)-\mathrm{B}(3)$ & $110.73(15)$ & $\mathrm{C}(2)-\mathrm{B}(7)-\mathrm{B}(11)$ & $104.54(15)$ \\
\hline $\mathrm{C}(1)-\mathrm{B}(4)-\mathrm{H}(4)$ & 124.2 & $\mathrm{C}(2)-\mathrm{B}(7)-\mathrm{B}(12)$ & $103.65(15)$ \\
\hline $\mathrm{B}(10)-\mathrm{B}(4)-\mathrm{H}(4)$ & 121.4 & $\mathrm{~B}(11)-\mathrm{B}(7)-\mathrm{B}(12)$ & $61.09(13)$ \\
\hline B(9)-B(4)-H(4) & 121.8 & $\mathrm{C}(2)-\mathrm{B}(7)-\mathrm{B}(6)$ & $59.06(12)$ \\
\hline $\mathrm{B}(5)-\mathrm{B}(4)-\mathrm{H}(4)$ & 120.3 & $\mathrm{~B}(11)-\mathrm{B}(7)-\mathrm{B}(6)$ & $59.84(12)$ \\
\hline $\mathrm{B}(3)-\mathrm{B}(4)-\mathrm{H}(4)$ & 119.8 & $\mathrm{~B}(12)-\mathrm{B}(7)-\mathrm{B}(6)$ & $108.56(16)$ \\
\hline $\mathrm{C}(1)-\mathrm{B}(5)-\mathrm{B}(11)$ & $104.87(15)$ & $\mathrm{C}(2)-\mathrm{B}(7)-\mathrm{B}(8)$ & $57.95(12)$ \\
\hline $\mathrm{C}(1)-\mathrm{B}(5)-\mathrm{B}(6)$ & $59.26(12)$ & $\mathrm{B}(11)-\mathrm{B}(7)-\mathrm{B}(8)$ & $108.97(16)$ \\
\hline $\mathrm{B}(11)-\mathrm{B}(5)-\mathrm{B}(6)$ & $60.06(12)$ & $\mathrm{B}(12)-\mathrm{B}(7)-\mathrm{B}(8)$ & $59.83(13)$ \\
\hline $\mathrm{C}(1)-\mathrm{B}(5)-\mathrm{B}(10)$ & 103.69(16) & $\mathrm{B}(6)-\mathrm{B}(7)-\mathrm{B}(8)$ & $108.37(15)$ \\
\hline $\mathrm{B}(11)-\mathrm{B}(5)-\mathrm{B}(10)$ & $60.67(13)$ & $\mathrm{C}(2)-\mathrm{B}(7)-\mathrm{H}(7)$ & 125.6 \\
\hline $\mathrm{B}(6)-\mathrm{B}(5)-\mathrm{B}(10)$ & 108.38(16) & $\mathrm{B}(11)-\mathrm{B}(7)-\mathrm{H}(7)$ & 121.8 \\
\hline $\mathrm{C}(1)-\mathrm{B}(5)-\mathrm{B}(4)$ & $57.78(11)$ & $\mathrm{B}(12)-\mathrm{B}(7)-\mathrm{H}(7)$ & 122.3 \\
\hline $\mathrm{B}(11)-\mathrm{B}(5)-\mathrm{B}(4)$ & 107.71(16) & $\mathrm{B}(6)-\mathrm{B}(7)-\mathrm{H}(7)$ & 121.1 \\
\hline $\mathrm{B}(6)-\mathrm{B}(5)-\mathrm{B}(4)$ & $107.70(15)$ & $\mathrm{B}(8)-\mathrm{B}(7)-\mathrm{H}(7)$ & 121.3 \\
\hline $\mathrm{B}(10)-\mathrm{B}(5)-\mathrm{B}(4)$ & $59.17(13)$ & $\mathrm{C}(2)-\mathrm{B}(8)-\mathrm{B}(9)$ & $104.44(15)$ \\
\hline $\mathrm{C}(1)-\mathrm{B}(5)-\mathrm{H}(5)$ & 125.1 & $\mathrm{C}(2)-\mathrm{B}(8)-\mathrm{B}(12)$ & $103.09(16)$ \\
\hline $\mathrm{B}(11)-\mathrm{B}(5)-\mathrm{H}(5)$ & 122.0 & $\mathrm{~B}(9)-\mathrm{B}(8)-\mathrm{B}(12)$ & $60.35(13)$ \\
\hline $\mathrm{B}(6)-\mathrm{B}(5)-\mathrm{H}(5)$ & 121.1 & $\mathrm{C}(2)-\mathrm{B}(8)-\mathrm{B}(3)$ & $60.28(12)$ \\
\hline $\mathrm{B}(10)-\mathrm{B}(5)-\mathrm{H}(5)$ & 122.6 & $\mathrm{~B}(9)-\mathrm{B}(8)-\mathrm{B}(3)$ & $60.61(12)$ \\
\hline $\mathrm{B}(4)-\mathrm{B}(5)-\mathrm{H}(5)$ & 122.2 & $\mathrm{~B}(12)-\mathrm{B}(8)-\mathrm{B}(3)$ & $109.68(16)$ \\
\hline $\mathrm{C}(2)-\mathrm{B}(6)-\mathrm{C}(1)$ & $56.74(11)$ & $\mathrm{C}(2)-\mathrm{B}(8)-\mathrm{B}(7)$ & $57.59(12)$ \\
\hline $\mathrm{C}(2)-\mathrm{B}(6)-\mathrm{B}(5)$ & $103.84(15)$ & $\mathrm{B}(9)-\mathrm{B}(8)-\mathrm{B}(7)$ & $107.95(16)$ \\
\hline $\mathrm{C}(1)-\mathrm{B}(6)-\mathrm{B}(5)$ & $58.89(12)$ & $\mathrm{B}(12)-\mathrm{B}(8)-\mathrm{B}(7)$ & $59.44(12)$ \\
\hline $\mathrm{C}(2)-\mathrm{B}(6)-\mathrm{B}(11)$ & $103.13(15)$ & $\mathrm{B}(3)-\mathrm{B}(8)-\mathrm{B}(7)$ & $109.75(15)$ \\
\hline $\mathrm{C}(1)-\mathrm{B}(6)-\mathrm{B}(11)$ & $104.52(15)$ & $\mathrm{C}(2)-\mathrm{B}(8)-\mathrm{H}(8)$ & 125.6 \\
\hline $\mathrm{B}(5)-\mathrm{B}(6)-\mathrm{B}(11)$ & $59.97(12)$ & $\mathrm{B}(9)-\mathrm{B}(8)-\mathrm{H}(8)$ & 122.3 \\
\hline $\mathrm{C}(2)-\mathrm{B}(6)-\mathrm{B}(7)$ & $57.46(12)$ & $\mathrm{B}(12)-\mathrm{B}(8)-\mathrm{H}(8)$ & 122.6 \\
\hline
\end{tabular}




\begin{tabular}{|c|c|c|c|}
\hline $\mathrm{B}(3)-\mathrm{B}(8)-\mathrm{H}(8)$ & 119.4 & $\mathrm{~B}(5)-\mathrm{B}(11)-\mathrm{B}(10)$ & $59.98(13)$ \\
\hline $\mathrm{B}(7)-\mathrm{B}(8)-\mathrm{H}(8)$ & 121.6 & $\mathrm{~B}(6)-\mathrm{B}(11)-\mathrm{B}(10)$ & $107.76(15)$ \\
\hline $\mathrm{B}(8)-\mathrm{B}(9)-\mathrm{B}(4)$ & $107.37(15)$ & $\mathrm{B}(7)-\mathrm{B}(11)-\mathrm{B}(12)$ & $59.63(13)$ \\
\hline $\mathrm{B}(8)-\mathrm{B}(9)-\mathrm{B}(12)$ & $60.09(13)$ & $\mathrm{B}(5)-\mathrm{B}(11)-\mathrm{B}(12)$ & $107.35(16)$ \\
\hline $\mathrm{B}(4)-\mathrm{B}(9)-\mathrm{B}(12)$ & $106.38(16)$ & $\mathrm{B}(6)-\mathrm{B}(11)-\mathrm{B}(12)$ & $107.98(16)$ \\
\hline $\mathrm{B}(8)-\mathrm{B}(9)-\mathrm{B}(10)$ & $107.41(16)$ & $\mathrm{B}(10)-\mathrm{B}(11)-\mathrm{B}(12)$ & $59.10(13)$ \\
\hline $\mathrm{B}(4)-\mathrm{B}(9)-\mathrm{B}(10)$ & $59.05(13)$ & $\mathrm{B}(7)-\mathrm{B}(11)-\mathrm{H}(11)$ & 121.8 \\
\hline $\mathrm{B}(12)-\mathrm{B}(9)-\mathrm{B}(10)$ & $59.29(13)$ & $\mathrm{B}(5)-\mathrm{B}(11)-\mathrm{H}(11)$ & 121.8 \\
\hline $\mathrm{B}(8)-\mathrm{B}(9)-\mathrm{B}(3)$ & $60.32(12)$ & $\mathrm{B}(6)-\mathrm{B}(11)-\mathrm{H}(11)$ & 121.4 \\
\hline $\mathrm{B}(4)-\mathrm{B}(9)-\mathrm{B}(3)$ & $61.25(12)$ & $\mathrm{B}(10)-\mathrm{B}(11)-\mathrm{H}(11)$ & 122.4 \\
\hline $\mathrm{B}(12)-\mathrm{B}(9)-\mathrm{B}(3)$ & $109.23(15)$ & $\mathrm{B}(12)-\mathrm{B}(11)-\mathrm{H}(11)$ & 122.3 \\
\hline $\mathrm{B}(10)-\mathrm{B}(9)-\mathrm{B}(3)$ & $109.43(15)$ & $\mathrm{B}(10)-\mathrm{B}(12)-\mathrm{B}(7)$ & $107.69(16)$ \\
\hline $\mathrm{B}(8)-\mathrm{B}(9)-\mathrm{H}(9)$ & 122.1 & $\mathrm{~B}(10)-\mathrm{B}(12)-\mathrm{B}(8)$ & $108.11(16)$ \\
\hline $\mathrm{B}(4)-\mathrm{B}(9)-\mathrm{H}(9)$ & 122.5 & $\mathrm{~B}(7)-\mathrm{B}(12)-\mathrm{B}(8)$ & $60.73(12)$ \\
\hline $\mathrm{B}(12)-\mathrm{B}(9)-\mathrm{H}(9)$ & 122.1 & $\mathrm{~B}(10)-\mathrm{B}(12)-\mathrm{B}(9)$ & $60.69(13)$ \\
\hline $\mathrm{B}(10)-\mathrm{B}(9)-\mathrm{H}(9)$ & 122.0 & $\mathrm{~B}(7)-\mathrm{B}(12)-\mathrm{B}(9)$ & $108.36(15)$ \\
\hline $\mathrm{B}(3)-\mathrm{B}(9)-\mathrm{H}(9)$ & 120.0 & $\mathrm{~B}(8)-\mathrm{B}(12)-\mathrm{B}(9)$ & $59.57(13)$ \\
\hline $\mathrm{B}(4)-\mathrm{B}(10)-\mathrm{B}(12)$ & $107.55(16)$ & $\mathrm{B}(10)-\mathrm{B}(12)-\mathrm{B}(11)$ & $60.28(13)$ \\
\hline $\mathrm{B}(4)-\mathrm{B}(10)-\mathrm{B}(5)$ & $60.46(12)$ & $\mathrm{B}(7)-\mathrm{B}(12)-\mathrm{B}(11)$ & $59.28(12)$ \\
\hline $\mathrm{B}(12)-\mathrm{B}(10)-\mathrm{B}(5)$ & $108.05(15)$ & $\mathrm{B}(8)-\mathrm{B}(12)-\mathrm{B}(11)$ & $108.25(15)$ \\
\hline $\mathrm{B}(4)-\mathrm{B}(10)-\mathrm{B}(11)$ & $107.69(15)$ & $\mathrm{B}(9)-\mathrm{B}(12)-\mathrm{B}(11)$ & $108.93(16)$ \\
\hline $\mathrm{B}(12)-\mathrm{B}(10)-\mathrm{B}(11)$ & $60.61(13)$ & $\mathrm{B}(10)-\mathrm{B}(12)-\mathrm{H}(12)$ & 121.6 \\
\hline $\mathrm{B}(5)-\mathrm{B}(10)-\mathrm{B}(11)$ & $59.35(13)$ & $\mathrm{B}(7)-\mathrm{B}(12)-\mathrm{H}(12)$ & 121.8 \\
\hline $\mathrm{B}(4)-\mathrm{B}(10)-\mathrm{B}(9)$ & $59.80(13)$ & $\mathrm{B}(8)-\mathrm{B}(12)-\mathrm{H}(12)$ & 121.6 \\
\hline $\mathrm{B}(12)-\mathrm{B}(10)-\mathrm{B}(9)$ & $60.01(13)$ & $\mathrm{B}(9)-\mathrm{B}(12)-\mathrm{H}(12)$ & 121.2 \\
\hline $\mathrm{B}(5)-\mathrm{B}(10)-\mathrm{B}(9)$ & $108.62(15)$ & $\mathrm{B}(11)-\mathrm{B}(12)-\mathrm{H}(12)$ & 121.5 \\
\hline $\mathrm{B}(11)-\mathrm{B}(10)-\mathrm{B}(9)$ & $108.65(16)$ & $\mathrm{C}(4)-\mathrm{P}(1)-\mathrm{C}(3)$ & $111.18(9)$ \\
\hline $\mathrm{B}(4)-\mathrm{B}(10)-\mathrm{H}(10)$ & 122.0 & $\mathrm{C}(4)-\mathrm{P}(1)-\mathrm{C}(1)$ & $105.50(9)$ \\
\hline $\mathrm{B}(12)-\mathrm{B}(10)-\mathrm{H}(10)$ & 121.7 & $\mathrm{C}(3)-\mathrm{P}(1)-\mathrm{C}(1)$ & 108.09(9) \\
\hline $\mathrm{B}(5)-\mathrm{B}(10)-\mathrm{H}(10)$ & 121.6 & $\mathrm{C}(4)-\mathrm{P}(1)-\operatorname{Ir}(1)$ & $121.64(7)$ \\
\hline $\mathrm{B}(11)-\mathrm{B}(10)-\mathrm{H}(10)$ & 121.7 & $\mathrm{C}(3)-\mathrm{P}(1)-\operatorname{Ir}(1)$ & $114.68(7)$ \\
\hline $\mathrm{B}(9)-\mathrm{B}(10)-\mathrm{H}(10)$ & 121.3 & $\mathrm{C}(1)-\mathrm{P}(1)-\operatorname{Ir}(1)$ & $92.68(6)$ \\
\hline $\mathrm{B}(7)-\mathrm{B}(11)-\mathrm{B}(5)$ & $108.08(15)$ & $\mathrm{C}(6)-\mathrm{C}(3)-\mathrm{C}(5)$ & $110.35(17)$ \\
\hline $\mathrm{B}(7)-\mathrm{B}(11)-\mathrm{B}(6)$ & $60.57(12)$ & $\mathrm{C}(6)-\mathrm{C}(3)-\mathrm{P}(1)$ & $117.42(14)$ \\
\hline $\mathrm{B}(5)-\mathrm{B}(11)-\mathrm{B}(6)$ & $59.97(12)$ & $\mathrm{C}(5)-\mathrm{C}(3)-\mathrm{P}(1)$ & $110.36(14)$ \\
\hline $\mathrm{B}(7)-\mathrm{B}(11)-\mathrm{B}(10)$ & $107.02(16)$ & $\mathrm{C}(6)-\mathrm{C}(3)-\mathrm{H}(3 \mathrm{~A})$ & 106.0 \\
\hline
\end{tabular}




$\begin{array}{llll}\mathrm{C}(5)-\mathrm{C}(3)-\mathrm{H}(3 \mathrm{~A}) & 106.0 & \mathrm{H}(6 \mathrm{~A})-\mathrm{C}(6)-\mathrm{H}(6 \mathrm{~B}) & 109.5 \\ \mathrm{P}(1)-\mathrm{C}(3)-\mathrm{H}(3 \mathrm{~A}) & 106.0 & \mathrm{C}(3)-\mathrm{C}(6)-\mathrm{H}(6 \mathrm{C}) & 109.5 \\ \mathrm{C}(8)-\mathrm{C}(4)-\mathrm{C}(7) & 111.76(16) & \mathrm{H}(6 \mathrm{~A})-\mathrm{C}(6)-\mathrm{H}(6 \mathrm{C}) & 109.5 \\ \mathrm{C}(8)-\mathrm{C}(4)-\mathrm{P}(1) & 111.57(14) & \mathrm{H}(6 \mathrm{~B})-\mathrm{C}(6)-\mathrm{H}(6 \mathrm{C}) & 109.5 \\ \mathrm{C}(7)-\mathrm{C}(4)-\mathrm{P}(1) & 115.10(14) & \mathrm{C}(4)-\mathrm{C}(7)-\mathrm{H}(7 \mathrm{~A}) & 109.5 \\ \mathrm{C}(8)-\mathrm{C}(4)-\mathrm{H}(4 \mathrm{~A}) & 105.9 & \mathrm{C}(4)-\mathrm{C}(7)-\mathrm{H}(7 \mathrm{~B}) & 109.5 \\ \mathrm{C}(7)-\mathrm{C}(4)-\mathrm{H}(4 \mathrm{~A}) & 105.9 & \mathrm{H}(7 \mathrm{~A})-\mathrm{C}(7)-\mathrm{H}(7 \mathrm{~B}) & 109.5 \\ \mathrm{P}(1)-\mathrm{C}(4)-\mathrm{H}(4 \mathrm{~A}) & 105.9 & \mathrm{C}(4)-\mathrm{C}(7)-\mathrm{H}(7 \mathrm{C}) & 109.5 \\ \mathrm{C}(3)-\mathrm{C}(5)-\mathrm{H}(5 \mathrm{~A}) & 109.5 & \mathrm{H}(7 \mathrm{~A})-\mathrm{C}(7)-\mathrm{H}(7 \mathrm{C}) & 109.5 \\ \mathrm{C}(3)-\mathrm{C}(5)-\mathrm{H}(5 \mathrm{~B}) & 109.5 & \mathrm{H}(7 \mathrm{~B})-\mathrm{C}(7)-\mathrm{H}(7 \mathrm{C}) & 109.5 \\ \mathrm{H}(5 \mathrm{~A})-\mathrm{C}(5)-\mathrm{H}(5 \mathrm{~B}) & 109.5 & \mathrm{C}(4)-\mathrm{C}(8)-\mathrm{H}(8 \mathrm{~A}) & 109.5 \\ \mathrm{C}(3)-\mathrm{C}(5)-\mathrm{H}(5 \mathrm{C}) & 109.5 & \mathrm{C}(4)-\mathrm{C}(8)-\mathrm{H}(8 \mathrm{~B}) & 109.5 \\ \mathrm{H}(5 \mathrm{~A})-\mathrm{C}(5)-\mathrm{H}(5 \mathrm{C}) & 109.5 & \mathrm{H}(8 \mathrm{~A})-\mathrm{C}(8)-\mathrm{H}(8 \mathrm{~B}) & 109.5 \\ \mathrm{H}(5 \mathrm{~B})-\mathrm{C}(5)-\mathrm{H}(5 \mathrm{C}) & 109.5 & \mathrm{C}(4)-\mathrm{C}(8)-\mathrm{H}(8 \mathrm{C}) & 109.5 \\ \mathrm{C}(3)-\mathrm{C}(6)-\mathrm{H}(6 \mathrm{~A}) & 109.5 & \mathrm{H}(8 \mathrm{~A})-\mathrm{C}(8)-\mathrm{H}(8 \mathrm{C}) & 109.5 \\ \mathrm{C}(3)-\mathrm{C}(6)-\mathrm{H}(6 \mathrm{~B}) & 109.5 & \mathrm{H}(8 \mathrm{~B})-\mathrm{C}(8)-\mathrm{H}(8 \mathrm{C}) & 109.5\end{array}$

Symmetry transformations used to generate equivalent atoms: 
Table 4. Anisotropic displacement parameters $\left(\AA^{2} \times 10^{3}\right)$ for vL67_0m. The anisotropic displacement factor exponent takes the form: $-2 \pi^{2}\left[h^{2} a^{* 2} U^{11}+\ldots+2 h k a^{*} b^{*} U^{12}\right]$

\begin{tabular}{|c|c|c|c|c|c|c|}
\hline & $\mathrm{U}^{11}$ & $\mathrm{U}^{22}$ & $\mathrm{U}^{33}$ & $\mathrm{U}^{23}$ & $\mathrm{U}^{13}$ & $\mathrm{U}^{12}$ \\
\hline $\operatorname{Ir}(1)$ & $10(1)$ & $7(1)$ & $9(1)$ & $0(1)$ & $4(1)$ & $-1(1)$ \\
\hline $\mathrm{Cl}(1)$ & $12(1)$ & $13(1)$ & $20(1)$ & $0(1)$ & $9(1)$ & $0(1)$ \\
\hline $\mathrm{C}(1 \mathrm{C})$ & $25(1)$ & $16(1)$ & $15(1)$ & $-6(1)$ & $7(1)$ & $-2(1)$ \\
\hline $\mathrm{C}(2 \mathrm{C})$ & $23(1)$ & $18(1)$ & $17(1)$ & $-7(1)$ & $6(1)$ & $-9(1)$ \\
\hline$C(3 C)$ & $60(2)$ & $59(2)$ & $36(2)$ & $-26(1)$ & $30(2)$ & $-47(2)$ \\
\hline $\mathrm{C}(4 \mathrm{C})$ & $31(1)$ & $29(1)$ & $33(1)$ & $9(1)$ & $8(1)$ & $-14(1)$ \\
\hline $\mathrm{C}(5 \mathrm{C})$ & $27(1)$ & $12(1)$ & $17(1)$ & $3(1)$ & $12(1)$ & $-4(1)$ \\
\hline$C(6 C)$ & $24(1)$ & $12(1)$ & $14(1)$ & $5(1)$ & $4(1)$ & $-1(1)$ \\
\hline $\mathrm{C}(7 \mathrm{C})$ & $42(2)$ & $21(1)$ & $31(1)$ & $10(1)$ & $19(1)$ & $13(1)$ \\
\hline $\mathrm{C}(8 \mathrm{C})$ & $54(2)$ & $35(2)$ & $31(1)$ & $-14(1)$ & $-6(1)$ & $27(1)$ \\
\hline $\mathrm{C}(1)$ & $10(1)$ & $10(1)$ & $10(1)$ & $0(1)$ & $3(1)$ & $0(1)$ \\
\hline $\mathrm{C}(2)$ & $10(1)$ & $10(1)$ & $10(1)$ & $0(1)$ & $4(1)$ & $-1(1)$ \\
\hline $\mathrm{B}(3)$ & $12(1)$ & $10(1)$ & $10(1)$ & $1(1)$ & $6(1)$ & $-1(1)$ \\
\hline $\mathrm{B}(4)$ & $12(1)$ & $13(1)$ & $13(1)$ & $-2(1)$ & $7(1)$ & $-2(1)$ \\
\hline B(5) & $16(1)$ & $14(1)$ & $15(1)$ & $-2(1)$ & $7(1)$ & $1(1)$ \\
\hline $\mathrm{B}(6)$ & $15(1)$ & $10(1)$ & $14(1)$ & $-1(1)$ & $6(1)$ & $-2(1)$ \\
\hline $\mathrm{B}(7)$ & $13(1)$ & $13(1)$ & 11(1) & $-3(1)$ & $3(1)$ & $-3(1)$ \\
\hline $\mathrm{B}(8)$ & $15(1)$ & $15(1)$ & $10(1)$ & $0(1)$ & $4(1)$ & $-3(1)$ \\
\hline $\mathrm{B}(9)$ & $16(1)$ & $16(1)$ & $12(1)$ & $-1(1)$ & $7(1)$ & $-3(1)$ \\
\hline $\mathrm{B}(10)$ & $15(1)$ & $18(1)$ & 12(1) & $-4(1)$ & $7(1)$ & $-2(1)$ \\
\hline $\mathrm{B}(11)$ & $16(1)$ & $15(1)$ & $12(1)$ & $-4(1)$ & $7(1)$ & $-3(1)$ \\
\hline $\mathrm{B}(12)$ & $15(1)$ & $17(1)$ & 12(1) & $-4(1)$ & $6(1)$ & $-4(1)$ \\
\hline $\mathrm{P}(1)$ & $9(1)$ & $9(1)$ & $8(1)$ & $0(1)$ & $3(1)$ & $-1(1)$ \\
\hline $\mathrm{C}(3)$ & 11(1) & $15(1)$ & 12(1) & $1(1)$ & $2(1)$ & 1(1) \\
\hline $\mathrm{C}(4)$ & $14(1)$ & $13(1)$ & $10(1)$ & $2(1)$ & $5(1)$ & $-1(1)$ \\
\hline $\mathrm{C}(5)$ & $15(1)$ & $25(1)$ & $13(1)$ & $-3(1)$ & $1(1)$ & $-2(1)$ \\
\hline $\mathrm{C}(6)$ & $14(1)$ & $20(1)$ & $19(1)$ & $2(1)$ & $3(1)$ & $3(1)$ \\
\hline $\mathrm{C}(7)$ & $23(1)$ & $14(1)$ & $17(1)$ & $4(1)$ & $9(1)$ & $-1(1)$ \\
\hline $\mathrm{C}(8)$ & $23(1)$ & $18(1)$ & $15(1)$ & $0(1)$ & $12(1)$ & $1(1)$ \\
\hline
\end{tabular}


Table 5. Hydrogen coordinates ( $\times 10^{4}$ ) and isotropic displacement parameters $\left(\AA^{2} \times 10^{3}\right)$ for vL67_0m.

\begin{tabular}{|c|c|c|c|c|}
\hline & $\mathrm{x}$ & $\mathrm{y}$ & $\mathrm{z}$ & $\mathrm{U}(\mathrm{eq})$ \\
\hline $\mathrm{H}(1)$ & $3518(18)$ & $1810(20)$ & $90(14)$ & 10 \\
\hline $\mathrm{H}(1 \mathrm{C})$ & $2240(20)$ & $3120(20)$ & $1376(17)$ & $23(7)$ \\
\hline $\mathrm{H}(2 \mathrm{C})$ & $3940(20)$ & $2790(20)$ & 1536(17) & $24(7)$ \\
\hline $\mathrm{H}(3 \mathrm{~B})$ & 4816 & 3703 & 835 & 58 \\
\hline $\mathrm{H}(3 \mathrm{C})$ & 4218 & 4907 & 950 & 58 \\
\hline $\mathrm{H}(4 \mathrm{~B})$ & 4178 & 4194 & -472 & 38 \\
\hline $\mathrm{H}(4 \mathrm{C})$ & 3302 & 5083 & -373 & 38 \\
\hline $\mathrm{H}(5 \mathrm{D})$ & $2870(20)$ & $2880(20)$ & $-1200(17)$ & $27(7)$ \\
\hline $\mathrm{H}(6 \mathrm{D})$ & $1150(20)$ & $3040(20)$ & $-1291(17)$ & $24(7)$ \\
\hline $\mathrm{H}(7 \mathrm{D})$ & 1283 & 5215 & -698 & 36 \\
\hline $\mathrm{H}(7 \mathrm{E})$ & 491 & 4239 & -559 & 36 \\
\hline $\mathrm{H}(8 \mathrm{D})$ & 1253 & 4758 & 734 & 56 \\
\hline $\mathrm{H}(8 \mathrm{E})$ & 2271 & 5309 & 613 & 56 \\
\hline $\mathrm{H}(2)$ & $833(18)$ & $-350(20)$ & $-526(14)$ & 12 \\
\hline $\mathrm{H}(4)$ & 4103 & -100 & -497 & 15 \\
\hline $\mathrm{H}(5)$ & 3618 & -2692 & -297 & 17 \\
\hline $\mathrm{H}(6)$ & 1472 & -2782 & -257 & 15 \\
\hline $\mathrm{H}(7)$ & -67 & -1924 & -1923 & 15 \\
\hline $\mathrm{H}(8)$ & 415 & 692 & -2113 & 16 \\
\hline $\mathrm{H}(9)$ & 2578 & 812 & -2146 & 17 \\
\hline $\mathrm{H}(10)$ & 3423 & -1739 & -1966 & 17 \\
\hline $\mathrm{H}(11)$ & 1779 & -3436 & -1822 & 17 \\
\hline $\mathrm{H}(12)$ & 1145 & -1266 & -2969 & 17 \\
\hline $\mathrm{H}(3 \mathrm{~A})$ & 4712 & 372 & 1116 & 15 \\
\hline $\mathrm{H}(4 \mathrm{~A})$ & 1354 & -703 & 883 & 14 \\
\hline $\mathrm{H}(5 \mathrm{~A})$ & 4419 & -340 & 2601 & 29 \\
\hline $\mathrm{H}(5 \mathrm{~B})$ & 4302 & 1054 & 2282 & 29 \\
\hline $\mathrm{H}(5 \mathrm{C})$ & 5426 & 366 & 2551 & 29 \\
\hline $\mathrm{H}(6 \mathrm{~A})$ & 5553 & -1485 & 1739 & 28 \\
\hline $\mathrm{H}(6 \mathrm{~B})$ & 4717 & -1680 & 793 & 28 \\
\hline
\end{tabular}




\begin{tabular}{lrrrr}
$\mathrm{H}(6 \mathrm{C})$ & 4408 & -2074 & 1564 & 28 \\
$\mathrm{H}(7 \mathrm{~A})$ & 3095 & -1905 & 2153 & 27 \\
$\mathrm{H}(7 \mathrm{~B})$ & 2520 & -2448 & 1223 & 27 \\
$\mathrm{H}(7 \mathrm{C})$ & 1865 & -2296 & 1813 & 27 \\
$\mathrm{H}(8 \mathrm{~A})$ & 1494 & -68 & 2195 & 26 \\
$\mathrm{H}(8 \mathrm{~B})$ & 1830 & 1061 & 1756 & 26 \\
$\mathrm{H}(8 \mathrm{C})$ & 2727 & 274 & 2465 & 26 \\
\hline
\end{tabular}


Table 6. Torsion angles $\left[^{\circ}\right]$ for vL67_0m.

\begin{tabular}{|c|c|}
\hline $\mathrm{C}(8 \mathrm{C})-\mathrm{C}(1 \mathrm{C})-\mathrm{C}(2 \mathrm{C})-\mathrm{C}(3 \mathrm{C})$ & $-1.6(4)$ \\
\hline $\operatorname{Ir}(1)-C(1 C)-C(2 C)-C(3 C)$ & 101.0(2) \\
\hline $\mathrm{C}(8 \mathrm{C})-\mathrm{C}(1 \mathrm{C})-\mathrm{C}(2 \mathrm{C})-\operatorname{Ir}(1)$ & $-102.6(2)$ \\
\hline$C(1 C)-C(2 C)-C(3 C)-C(4 C)$ & $-55.5(4)$ \\
\hline $\operatorname{Ir}(1)-C(2 C)-C(3 C)-C(4 C)$ & $27.5(3)$ \\
\hline $\mathrm{C}(2 \mathrm{C})-\mathrm{C}(3 \mathrm{C})-\mathrm{C}(4 \mathrm{C})-\mathrm{C}(5 \mathrm{C})$ & $-18.1(4)$ \\
\hline$C(3 C)-C(4 C)-C(5 C)-C(6 C)$ & $79.7(3)$ \\
\hline$C(3 C)-C(4 C)-C(5 C)-\operatorname{Ir}(1)$ & $-2.3(3)$ \\
\hline$C(4 C)-C(5 C)-C(6 C)-C(7 C)$ & $0.0(3)$ \\
\hline $\operatorname{Ir}(1)-C(5 C)-C(6 C)-C(7 C)$ & $105.0(2)$ \\
\hline$C(4 C)-C(5 C)-C(6 C)-\operatorname{Ir}(1)$ & $-105.0(2)$ \\
\hline $\mathrm{C}(5 \mathrm{C})-\mathrm{C}(6 \mathrm{C})-\mathrm{C}(7 \mathrm{C})-\mathrm{C}(8 \mathrm{C})$ & $-55.3(3)$ \\
\hline $\operatorname{Ir}(1)-C(6 C)-C(7 C)-C(8 C)$ & $28.4(3)$ \\
\hline $\mathrm{C}(6 \mathrm{C})-\mathrm{C}(7 \mathrm{C})-\mathrm{C}(8 \mathrm{C})-\mathrm{C}(1 \mathrm{C})$ & $-19.9(4)$ \\
\hline$C(2 C)-C(1 C)-C(8 C)-C(7 C)$ & $83.0(4)$ \\
\hline $\operatorname{Ir}(1)-C(1 C)-C(8 C)-C(7 C)$ & $1.6(4)$ \\
\hline $\mathrm{B}(4)-\mathrm{C}(1)-\mathrm{C}(2)-\mathrm{B}(7)$ & $67.70(19)$ \\
\hline $\mathrm{B}(5)-\mathrm{C}(1)-\mathrm{C}(2)-\mathrm{B}(7)$ & $0.2(2)$ \\
\hline $\mathrm{B}(6)-\mathrm{C}(1)-\mathrm{C}(2)-\mathrm{B}(7)$ & $-39.58(15)$ \\
\hline $\mathrm{B}(3)-\mathrm{C}(1)-\mathrm{C}(2)-\mathrm{B}(7)$ & $110.53(16)$ \\
\hline $\mathrm{P}(1)-\mathrm{C}(1)-\mathrm{C}(2)-\mathrm{B}(7)$ & $-168.66(12)$ \\
\hline $\mathrm{B}(4)-\mathrm{C}(1)-\mathrm{C}(2)-\mathrm{B}(8)$ & $-2.9(2)$ \\
\hline $\mathrm{B}(5)-\mathrm{C}(1)-\mathrm{C}(2)-\mathrm{B}(8)$ & $-70.39(19)$ \\
\hline $\mathrm{B}(6)-\mathrm{C}(1)-\mathrm{C}(2)-\mathrm{B}(8)$ & $-110.18(17)$ \\
\hline $\mathrm{B}(3)-\mathrm{C}(1)-\mathrm{C}(2)-\mathrm{B}(8)$ & $39.93(15)$ \\
\hline $\mathrm{P}(1)-\mathrm{C}(1)-\mathrm{C}(2)-\mathrm{B}(8)$ & $120.74(14)$ \\
\hline $\mathrm{B}(4)-\mathrm{C}(1)-\mathrm{C}(2)-\mathrm{B}(6)$ & $107.28(16)$ \\
\hline $\mathrm{B}(5)-\mathrm{C}(1)-\mathrm{C}(2)-\mathrm{B}(6)$ & $39.79(14)$ \\
\hline $\mathrm{B}(3)-\mathrm{C}(1)-\mathrm{C}(2)-\mathrm{B}(6)$ & $150.10(15)$ \\
\hline $\mathrm{P}(1)-\mathrm{C}(1)-\mathrm{C}(2)-\mathrm{B}(6)$ & $-129.08(14)$ \\
\hline $\mathrm{B}(4)-\mathrm{C}(1)-\mathrm{C}(2)-\mathrm{B}(3)$ & $-42.83(14)$ \\
\hline $\mathrm{B}(5)-\mathrm{C}(1)-\mathrm{C}(2)-\mathrm{B}(3)$ & $-110.32(16)$ \\
\hline $\mathrm{B}(6)-\mathrm{C}(1)-\mathrm{C}(2)-\mathrm{B}(3)$ & $-150.10(15)$ \\
\hline $\mathrm{P}(1)-\mathrm{C}(1)-\mathrm{C}(2)-\mathrm{B}(3)$ & $80.82(12)$ \\
\hline
\end{tabular}




\begin{tabular}{|c|c|}
\hline $\mathrm{B}(7)-\mathrm{C}(2)-\mathrm{B}(3)-\mathrm{C}(1)$ & $-102.87(17)$ \\
\hline $\mathrm{B}(8)-\mathrm{C}(2)-\mathrm{B}(3)-\mathrm{C}(1)$ & $-138.21(15)$ \\
\hline $\mathrm{B}(6)-\mathrm{C}(2)-\mathrm{B}(3)-\mathrm{C}(1)$ & $-29.89(15)$ \\
\hline $\mathrm{C}(1)-\mathrm{C}(2)-\mathrm{B}(3)-\mathrm{B}(8)$ & $138.21(15)$ \\
\hline $\mathrm{B}(7)-\mathrm{C}(2)-\mathrm{B}(3)-\mathrm{B}(8)$ & $35.35(16)$ \\
\hline $\mathrm{B}(6)-\mathrm{C}(2)-\mathrm{B}(3)-\mathrm{B}(8)$ & $108.32(18)$ \\
\hline $\mathrm{C}(1)-\mathrm{C}(2)-\mathrm{B}(3)-\mathrm{B}(9)$ & $96.71(15)$ \\
\hline $\mathrm{B}(7)-\mathrm{C}(2)-\mathrm{B}(3)-\mathrm{B}(9)$ & $-6.2(2)$ \\
\hline $\mathrm{B}(8)-\mathrm{C}(2)-\mathrm{B}(3)-\mathrm{B}(9)$ & $-41.51(14)$ \\
\hline $\mathrm{B}(6)-\mathrm{C}(2)-\mathrm{B}(3)-\mathrm{B}(9)$ & $66.82(19)$ \\
\hline $\mathrm{C}(1)-\mathrm{C}(2)-\mathrm{B}(3)-\mathrm{B}(4)$ & $37.04(12)$ \\
\hline $\mathrm{B}(7)-\mathrm{C}(2)-\mathrm{B}(3)-\mathrm{B}(4)$ & $-65.83(19)$ \\
\hline $\mathrm{B}(8)-\mathrm{C}(2)-\mathrm{B}(3)-\mathrm{B}(4)$ & $-101.18(15)$ \\
\hline $\mathrm{B}(6)-\mathrm{C}(2)-\mathrm{B}(3)-\mathrm{B}(4)$ & $7.15(19)$ \\
\hline $\mathrm{C}(1)-\mathrm{C}(2)-\mathrm{B}(3)-\operatorname{Ir}(1)$ & $-90.28(14)$ \\
\hline $\mathrm{B}(7)-\mathrm{C}(2)-\mathrm{B}(3)-\operatorname{Ir}(1)$ & $166.85(13)$ \\
\hline $\mathrm{B}(8)-\mathrm{C}(2)-\mathrm{B}(3)-\operatorname{Ir}(1)$ & 131.51(16) \\
\hline $\mathrm{B}(6)-\mathrm{C}(2)-\mathrm{B}(3)-\operatorname{Ir}(1)$ & $-120.17(15)$ \\
\hline $\mathrm{B}(4)-\mathrm{C}(1)-\mathrm{B}(3)-\mathrm{C}(2)$ & $134.41(15)$ \\
\hline $\mathrm{B}(5)-\mathrm{C}(1)-\mathrm{B}(3)-\mathrm{C}(2)$ & $99.16(17)$ \\
\hline $\mathrm{B}(6)-\mathrm{C}(1)-\mathrm{B}(3)-\mathrm{C}(2)$ & $29.14(14)$ \\
\hline $\mathrm{P}(1)-\mathrm{C}(1)-\mathrm{B}(3)-\mathrm{C}(2)$ & $-111.15(11)$ \\
\hline $\mathrm{C}(2)-\mathrm{C}(1)-\mathrm{B}(3)-\mathrm{B}(8)$ & $-34.72(13)$ \\
\hline $\mathrm{B}(4)-\mathrm{C}(1)-\mathrm{B}(3)-\mathrm{B}(8)$ & $99.68(15)$ \\
\hline $\mathrm{B}(5)-\mathrm{C}(1)-\mathrm{B}(3)-\mathrm{B}(8)$ & $64.44(18)$ \\
\hline $\mathrm{B}(6)-\mathrm{C}(1)-\mathrm{B}(3)-\mathrm{B}(8)$ & $-5.59(19)$ \\
\hline $\mathrm{P}(1)-\mathrm{C}(1)-\mathrm{B}(3)-\mathrm{B}(8)$ & $-145.88(12)$ \\
\hline $\mathrm{C}(2)-\mathrm{C}(1)-\mathrm{B}(3)-\mathrm{B}(9)$ & $-95.15(15)$ \\
\hline $\mathrm{B}(4)-\mathrm{C}(1)-\mathrm{B}(3)-\mathrm{B}(9)$ & $39.26(14)$ \\
\hline $\mathrm{B}(5)-\mathrm{C}(1)-\mathrm{B}(3)-\mathrm{B}(9)$ & $4.0(2)$ \\
\hline $\mathrm{B}(6)-\mathrm{C}(1)-\mathrm{B}(3)-\mathrm{B}(9)$ & $-66.01(18)$ \\
\hline $\mathrm{P}(1)-\mathrm{C}(1)-\mathrm{B}(3)-\mathrm{B}(9)$ & $153.70(12)$ \\
\hline $\mathrm{C}(2)-\mathrm{C}(1)-\mathrm{B}(3)-\mathrm{B}(4)$ & $-134.41(15)$ \\
\hline $\mathrm{B}(5)-\mathrm{C}(1)-\mathrm{B}(3)-\mathrm{B}(4)$ & $-35.25(15)$ \\
\hline $\mathrm{B}(6)-\mathrm{C}(1)-\mathrm{B}(3)-\mathrm{B}(4)$ & $-105.27(17)$ \\
\hline $\mathrm{P}(1)-\mathrm{C}(1)-\mathrm{B}(3)-\mathrm{B}(4)$ & $114.44(12)$ \\
\hline
\end{tabular}




\begin{tabular}{|c|c|}
\hline $\mathrm{C}(2)-\mathrm{C}(1)-\mathrm{B}(3)-\operatorname{Ir}(1)$ & $110.57(12)$ \\
\hline $\mathrm{B}(4)-\mathrm{C}(1)-\mathrm{B}(3)-\operatorname{Ir}(1)$ & $-115.03(13)$ \\
\hline $\mathrm{B}(5)-\mathrm{C}(1)-\mathrm{B}(3)-\operatorname{Ir}(1)$ & $-150.27(13)$ \\
\hline $\mathrm{B}(6)-\mathrm{C}(1)-\mathrm{B}(3)-\operatorname{Ir}(1)$ & $139.70(13)$ \\
\hline $\mathrm{P}(1)-\mathrm{C}(1)-\mathrm{B}(3)-\operatorname{Ir}(1)$ & $-0.59(11)$ \\
\hline $\mathrm{C}(2)-\mathrm{C}(1)-\mathrm{B}(4)-\mathrm{B}(10)$ & $-62.51(19)$ \\
\hline $\mathrm{B}(5)-\mathrm{C}(1)-\mathrm{B}(4)-\mathrm{B}(10)$ & $40.02(15)$ \\
\hline $\mathrm{B}(6)-\mathrm{C}(1)-\mathrm{B}(4)-\mathrm{B}(10)$ & $3.9(2)$ \\
\hline $\mathrm{B}(3)-\mathrm{C}(1)-\mathrm{B}(4)-\mathrm{B}(10)$ & $-104.58(17)$ \\
\hline $\mathrm{P}(1)-\mathrm{C}(1)-\mathrm{B}(4)-\mathrm{B}(10)$ & $175.70(13)$ \\
\hline $\mathrm{C}(2)-\mathrm{C}(1)-\mathrm{B}(4)-\mathrm{B}(9)$ & $1.3(2)$ \\
\hline $\mathrm{B}(5)-\mathrm{C}(1)-\mathrm{B}(4)-\mathrm{B}(9)$ & 103.81(17) \\
\hline $\mathrm{B}(6)-\mathrm{C}(1)-\mathrm{B}(4)-\mathrm{B}(9)$ & 67.74(19) \\
\hline $\mathrm{B}(3)-\mathrm{C}(1)-\mathrm{B}(4)-\mathrm{B}(9)$ & $-40.79(14)$ \\
\hline $\mathrm{P}(1)-\mathrm{C}(1)-\mathrm{B}(4)-\mathrm{B}(9)$ & $-120.50(14)$ \\
\hline $\mathrm{C}(2)-\mathrm{C}(1)-\mathrm{B}(4)-\mathrm{B}(5)$ & $-102.53(16)$ \\
\hline $\mathrm{B}(6)-\mathrm{C}(1)-\mathrm{B}(4)-\mathrm{B}(5)$ & $-36.07(16)$ \\
\hline $\mathrm{B}(3)-\mathrm{C}(1)-\mathrm{B}(4)-\mathrm{B}(5)$ & $-144.60(16)$ \\
\hline $\mathrm{P}(1)-\mathrm{C}(1)-\mathrm{B}(4)-\mathrm{B}(5)$ & $135.69(15)$ \\
\hline $\mathrm{C}(2)-\mathrm{C}(1)-\mathrm{B}(4)-\mathrm{B}(3)$ & $42.07(14)$ \\
\hline $\mathrm{B}(5)-\mathrm{C}(1)-\mathrm{B}(4)-\mathrm{B}(3)$ & $144.60(16)$ \\
\hline $\mathrm{B}(6)-\mathrm{C}(1)-\mathrm{B}(4)-\mathrm{B}(3)$ & $108.53(16)$ \\
\hline $\mathrm{P}(1)-\mathrm{C}(1)-\mathrm{B}(4)-\mathrm{B}(3)$ & $-79.71(13)$ \\
\hline $\mathrm{C}(2)-\mathrm{B}(3)-\mathrm{B}(4)-\mathrm{C}(1)$ & $-36.54(12)$ \\
\hline $\mathrm{B}(8)-\mathrm{B}(3)-\mathrm{B}(4)-\mathrm{C}(1)$ & $-94.54(15)$ \\
\hline $\mathrm{B}(9)-\mathrm{B}(3)-\mathrm{B}(4)-\mathrm{C}(1)$ & $-133.47(16)$ \\
\hline $\operatorname{Ir}(1)-B(3)-B(4)-C(1)$ & $88.24(14)$ \\
\hline $\mathrm{C}(2)-\mathrm{B}(3)-\mathrm{B}(4)-\mathrm{B}(10)$ & $60.51(17)$ \\
\hline $\mathrm{C}(1)-\mathrm{B}(3)-\mathrm{B}(4)-\mathrm{B}(10)$ & $97.05(16)$ \\
\hline $\mathrm{B}(8)-\mathrm{B}(3)-\mathrm{B}(4)-\mathrm{B}(10)$ & $2.51(19)$ \\
\hline $\mathrm{B}(9)-\mathrm{B}(3)-\mathrm{B}(4)-\mathrm{B}(10)$ & $-36.42(15)$ \\
\hline $\operatorname{Ir}(1)-\mathrm{B}(3)-\mathrm{B}(4)-\mathrm{B}(10)$ & $-174.72(13)$ \\
\hline $\mathrm{C}(2)-\mathrm{B}(3)-\mathrm{B}(4)-\mathrm{B}(9)$ & $96.94(15)$ \\
\hline $\mathrm{C}(1)-\mathrm{B}(3)-\mathrm{B}(4)-\mathrm{B}(9)$ & $133.47(16)$ \\
\hline $\mathrm{B}(8)-\mathrm{B}(3)-\mathrm{B}(4)-\mathrm{B}(9)$ & $38.93(14)$ \\
\hline $\operatorname{Ir}(1)-B(3)-B(4)-B(9)$ & $-138.29(16)$ \\
\hline
\end{tabular}




\begin{tabular}{|c|c|}
\hline $\mathrm{C}(2)-\mathrm{B}(3)-\mathrm{B}(4)-\mathrm{B}(5)$ & $-4.39(18)$ \\
\hline $\mathrm{C}(1)-\mathrm{B}(3)-\mathrm{B}(4)-\mathrm{B}(5)$ & $32.14(14)$ \\
\hline $\mathrm{B}(8)-\mathrm{B}(3)-\mathrm{B}(4)-\mathrm{B}(5)$ & $-62.39(18)$ \\
\hline $\mathrm{B}(9)-\mathrm{B}(3)-\mathrm{B}(4)-\mathrm{B}(5)$ & $-101.33(17)$ \\
\hline $\operatorname{Ir}(1)-B(3)-B(4)-B(5)$ & $120.38(15)$ \\
\hline $\mathrm{C}(2)-\mathrm{C}(1)-\mathrm{B}(5)-\mathrm{B}(11)$ & $1.16(19)$ \\
\hline $\mathrm{B}(4)-\mathrm{C}(1)-\mathrm{B}(5)-\mathrm{B}(11)$ & $-101.81(16)$ \\
\hline $\mathrm{B}(6)-\mathrm{C}(1)-\mathrm{B}(5)-\mathrm{B}(11)$ & $40.65(14)$ \\
\hline $\mathrm{B}(3)-\mathrm{C}(1)-\mathrm{B}(5)-\mathrm{B}(11)$ & $-66.41(19)$ \\
\hline $\mathrm{P}(1)-\mathrm{C}(1)-\mathrm{B}(5)-\mathrm{B}(11)$ & $164.80(17)$ \\
\hline $\mathrm{C}(2)-\mathrm{C}(1)-\mathrm{B}(5)-\mathrm{B}(6)$ & $-39.49(14)$ \\
\hline $\mathrm{B}(4)-\mathrm{C}(1)-\mathrm{B}(5)-\mathrm{B}(6)$ & $-142.46(16)$ \\
\hline $\mathrm{B}(3)-\mathrm{C}(1)-\mathrm{B}(5)-\mathrm{B}(6)$ & $-107.06(17)$ \\
\hline $\mathrm{P}(1)-\mathrm{C}(1)-\mathrm{B}(5)-\mathrm{B}(6)$ & $124.2(2)$ \\
\hline $\mathrm{C}(2)-\mathrm{C}(1)-\mathrm{B}(5)-\mathrm{B}(10)$ & $63.97(18)$ \\
\hline $\mathrm{B}(4)-\mathrm{C}(1)-\mathrm{B}(5)-\mathrm{B}(10)$ & $-39.00(14)$ \\
\hline $\mathrm{B}(6)-\mathrm{C}(1)-\mathrm{B}(5)-\mathrm{B}(10)$ & $103.46(16)$ \\
\hline $\mathrm{B}(3)-\mathrm{C}(1)-\mathrm{B}(5)-\mathrm{B}(10)$ & $-3.6(2)$ \\
\hline $\mathrm{P}(1)-\mathrm{C}(1)-\mathrm{B}(5)-\mathrm{B}(10)$ & $-132.39(19)$ \\
\hline $\mathrm{C}(2)-\mathrm{C}(1)-\mathrm{B}(5)-\mathrm{B}(4)$ & $102.97(16)$ \\
\hline $\mathrm{B}(6)-\mathrm{C}(1)-\mathrm{B}(5)-\mathrm{B}(4)$ & $142.46(16)$ \\
\hline $\mathrm{B}(3)-\mathrm{C}(1)-\mathrm{B}(5)-\mathrm{B}(4)$ & $35.40(16)$ \\
\hline $\mathrm{P}(1)-\mathrm{C}(1)-\mathrm{B}(5)-\mathrm{B}(4)$ & $-93.4(2)$ \\
\hline $\mathrm{B}(10)-\mathrm{B}(4)-\mathrm{B}(5)-\mathrm{C}(1)$ & $-134.60(16)$ \\
\hline $\mathrm{B}(9)-\mathrm{B}(4)-\mathrm{B}(5)-\mathrm{C}(1)$ & $-96.98(16)$ \\
\hline $\mathrm{B}(3)-\mathrm{B}(4)-\mathrm{B}(5)-\mathrm{C}(1)$ & $-32.53(14)$ \\
\hline $\mathrm{C}(1)-\mathrm{B}(4)-\mathrm{B}(5)-\mathrm{B}(11)$ & $96.73(16)$ \\
\hline $\mathrm{B}(10)-\mathrm{B}(4)-\mathrm{B}(5)-\mathrm{B}(11)$ & $-37.87(15)$ \\
\hline $\mathrm{B}(9)-\mathrm{B}(4)-\mathrm{B}(5)-\mathrm{B}(11)$ & $-0.2(2)$ \\
\hline $\mathrm{B}(3)-\mathrm{B}(4)-\mathrm{B}(5)-\mathrm{B}(11)$ & $64.20(19)$ \\
\hline $\mathrm{C}(1)-\mathrm{B}(4)-\mathrm{B}(5)-\mathrm{B}(6)$ & $33.35(14)$ \\
\hline $\mathrm{B}(10)-\mathrm{B}(4)-\mathrm{B}(5)-\mathrm{B}(6)$ & $-101.25(17)$ \\
\hline $\mathrm{B}(9)-\mathrm{B}(4)-\mathrm{B}(5)-\mathrm{B}(6)$ & $-63.63(19)$ \\
\hline $\mathrm{B}(3)-\mathrm{B}(4)-\mathrm{B}(5)-\mathrm{B}(6)$ & $0.8(2)$ \\
\hline $\mathrm{C}(1)-\mathrm{B}(4)-\mathrm{B}(5)-\mathrm{B}(10)$ & $134.60(16)$ \\
\hline $\mathrm{B}(9)-\mathrm{B}(4)-\mathrm{B}(5)-\mathrm{B}(10)$ & $37.62(15)$ \\
\hline
\end{tabular}




\begin{tabular}{|c|c|}
\hline $\mathrm{B}(3)-\mathrm{B}(4)-\mathrm{B}(5)-\mathrm{B}(10)$ & $102.06(17)$ \\
\hline $\mathrm{B}(7)-\mathrm{C}(2)-\mathrm{B}(6)-\mathrm{C}(1)$ & 138.97(15) \\
\hline $\mathrm{B}(8)-\mathrm{C}(2)-\mathrm{B}(6)-\mathrm{C}(1)$ & $102.27(17)$ \\
\hline $\mathrm{B}(3)-\mathrm{C}(2)-\mathrm{B}(6)-\mathrm{C}(1)$ & $30.12(15)$ \\
\hline $\mathrm{C}(1)-\mathrm{C}(2)-\mathrm{B}(6)-\mathrm{B}(5)$ & $-37.06(13)$ \\
\hline $\mathrm{B}(7)-\mathrm{C}(2)-\mathrm{B}(6)-\mathrm{B}(5)$ & 101.91(16) \\
\hline $\mathrm{B}(8)-\mathrm{C}(2)-\mathrm{B}(6)-\mathrm{B}(5)$ & $65.21(19)$ \\
\hline $\mathrm{B}(3)-\mathrm{C}(2)-\mathrm{B}(6)-\mathrm{B}(5)$ & $-6.9(2)$ \\
\hline $\mathrm{C}(1)-\mathrm{C}(2)-\mathrm{B}(6)-\mathrm{B}(11)$ & $-98.91(15)$ \\
\hline $\mathrm{B}(7)-\mathrm{C}(2)-\mathrm{B}(6)-\mathrm{B}(11)$ & $40.06(14)$ \\
\hline $\mathrm{B}(8)-\mathrm{C}(2)-\mathrm{B}(6)-\mathrm{B}(11)$ & $3.4(2)$ \\
\hline $\mathrm{B}(3)-\mathrm{C}(2)-\mathrm{B}(6)-\mathrm{B}(11)$ & $-68.79(19)$ \\
\hline $\mathrm{C}(1)-\mathrm{C}(2)-\mathrm{B}(6)-\mathrm{B}(7)$ & $-138.97(15)$ \\
\hline $\mathrm{B}(8)-\mathrm{C}(2)-\mathrm{B}(6)-\mathrm{B}(7)$ & $-36.70(16)$ \\
\hline $\mathrm{B}(3)-\mathrm{C}(2)-\mathrm{B}(6)-\mathrm{B}(7)$ & $-108.85(17)$ \\
\hline $\mathrm{B}(4)-\mathrm{C}(1)-\mathrm{B}(6)-\mathrm{C}(2)$ & $-100.37(16)$ \\
\hline $\mathrm{B}(5)-\mathrm{C}(1)-\mathrm{B}(6)-\mathrm{C}(2)$ & $-136.88(15)$ \\
\hline $\mathrm{B}(3)-\mathrm{C}(1)-\mathrm{B}(6)-\mathrm{C}(2)$ & $-29.34(14)$ \\
\hline $\mathrm{P}(1)-\mathrm{C}(1)-\mathrm{B}(6)-\mathrm{C}(2)$ & $90.06(18)$ \\
\hline $\mathrm{C}(2)-\mathrm{C}(1)-\mathrm{B}(6)-\mathrm{B}(5)$ & $136.88(15)$ \\
\hline $\mathrm{B}(4)-\mathrm{C}(1)-\mathrm{B}(6)-\mathrm{B}(5)$ & $36.52(16)$ \\
\hline $\mathrm{B}(3)-\mathrm{C}(1)-\mathrm{B}(6)-\mathrm{B}(5)$ & $107.54(16)$ \\
\hline $\mathrm{P}(1)-\mathrm{C}(1)-\mathrm{B}(6)-\mathrm{B}(5)$ & $-133.06(19)$ \\
\hline $\mathrm{C}(2)-\mathrm{C}(1)-\mathrm{B}(6)-\mathrm{B}(11)$ & $96.36(16)$ \\
\hline $\mathrm{B}(4)-\mathrm{C}(1)-\mathrm{B}(6)-\mathrm{B}(11)$ & $-4.0(2)$ \\
\hline $\mathrm{B}(5)-\mathrm{C}(1)-\mathrm{B}(6)-\mathrm{B}(11)$ & $-40.52(15)$ \\
\hline $\mathrm{B}(3)-\mathrm{C}(1)-\mathrm{B}(6)-\mathrm{B}(11)$ & $67.02(19)$ \\
\hline $\mathrm{P}(1)-\mathrm{C}(1)-\mathrm{B}(6)-\mathrm{B}(11)$ & $-173.58(15)$ \\
\hline $\mathrm{C}(2)-\mathrm{C}(1)-\mathrm{B}(6)-\mathrm{B}(7)$ & $34.72(13)$ \\
\hline $\mathrm{B}(4)-\mathrm{C}(1)-\mathrm{B}(6)-\mathrm{B}(7)$ & $-65.65(19)$ \\
\hline $\mathrm{B}(5)-\mathrm{C}(1)-\mathrm{B}(6)-\mathrm{B}(7)$ & $-102.17(16)$ \\
\hline $\mathrm{B}(3)-\mathrm{C}(1)-\mathrm{B}(6)-\mathrm{B}(7)$ & $5.38(19)$ \\
\hline $\mathrm{P}(1)-\mathrm{C}(1)-\mathrm{B}(6)-\mathrm{B}(7)$ & $124.77(16)$ \\
\hline $\mathrm{C}(1)-\mathrm{B}(5)-\mathrm{B}(6)-\mathrm{C}(2)$ & $36.06(13)$ \\
\hline $\mathrm{B}(11)-\mathrm{B}(5)-\mathrm{B}(6)-\mathrm{C}(2)$ & $-97.35(16)$ \\
\hline $\mathrm{B}(10)-\mathrm{B}(5)-\mathrm{B}(6)-\mathrm{C}(2)$ & $-59.26(19)$ \\
\hline
\end{tabular}




\begin{tabular}{|c|c|}
\hline $\mathrm{B}(4)-\mathrm{B}(5)-\mathrm{B}(6)-\mathrm{C}(2)$ & $3.30(19)$ \\
\hline $\mathrm{B}(11)-\mathrm{B}(5)-\mathrm{B}(6)-\mathrm{C}(1)$ & $-133.41(16)$ \\
\hline $\mathrm{B}(10)-\mathrm{B}(5)-\mathrm{B}(6)-\mathrm{C}(1)$ & $-95.31(17)$ \\
\hline $\mathrm{B}(4)-\mathrm{B}(5)-\mathrm{B}(6)-\mathrm{C}(1)$ & $-32.76(14)$ \\
\hline $\mathrm{C}(1)-\mathrm{B}(5)-\mathrm{B}(6)-\mathrm{B}(11)$ & 133.41(16) \\
\hline $\mathrm{B}(10)-\mathrm{B}(5)-\mathrm{B}(6)-\mathrm{B}(11)$ & $38.09(15)$ \\
\hline $\mathrm{B}(4)-\mathrm{B}(5)-\mathrm{B}(6)-\mathrm{B}(11)$ & $100.65(17)$ \\
\hline $\mathrm{C}(1)-\mathrm{B}(5)-\mathrm{B}(6)-\mathrm{B}(7)$ & $95.82(16)$ \\
\hline $\mathrm{B}(11)-\mathrm{B}(5)-\mathrm{B}(6)-\mathrm{B}(7)$ & $-37.58(15)$ \\
\hline $\mathrm{B}(10)-\mathrm{B}(5)-\mathrm{B}(6)-\mathrm{B}(7)$ & $0.5(2)$ \\
\hline $\mathrm{B}(4)-\mathrm{B}(5)-\mathrm{B}(6)-\mathrm{B}(7)$ & $63.07(19)$ \\
\hline $\mathrm{C}(1)-\mathrm{C}(2)-\mathrm{B}(7)-\mathrm{B}(11)$ & $-1.5(2)$ \\
\hline $\mathrm{B}(8)-\mathrm{C}(2)-\mathrm{B}(7)-\mathrm{B}(11)$ & $103.52(16)$ \\
\hline $\mathrm{B}(6)-\mathrm{C}(2)-\mathrm{B}(7)-\mathrm{B}(11)$ & $-40.47(14)$ \\
\hline $\mathrm{B}(3)-\mathrm{C}(2)-\mathrm{B}(7)-\mathrm{B}(11)$ & $68.7(2)$ \\
\hline $\mathrm{C}(1)-\mathrm{C}(2)-\mathrm{B}(7)-\mathrm{B}(12)$ & $-64.68(19)$ \\
\hline $\mathrm{B}(8)-\mathrm{C}(2)-\mathrm{B}(7)-\mathrm{B}(12)$ & $40.32(15)$ \\
\hline $\mathrm{B}(6)-\mathrm{C}(2)-\mathrm{B}(7)-\mathrm{B}(12)$ & $-103.67(16)$ \\
\hline $\mathrm{B}(3)-\mathrm{C}(2)-\mathrm{B}(7)-\mathrm{B}(12)$ & $5.5(2)$ \\
\hline $\mathrm{C}(1)-\mathrm{C}(2)-\mathrm{B}(7)-\mathrm{B}(6)$ & $38.99(14)$ \\
\hline $\mathrm{B}(8)-\mathrm{C}(2)-\mathrm{B}(7)-\mathrm{B}(6)$ & $143.99(15)$ \\
\hline $\mathrm{B}(3)-\mathrm{C}(2)-\mathrm{B}(7)-\mathrm{B}(6)$ & $109.20(17)$ \\
\hline $\mathrm{C}(1)-\mathrm{C}(2)-\mathrm{B}(7)-\mathrm{B}(8)$ & $-105.00(16)$ \\
\hline $\mathrm{B}(6)-\mathrm{C}(2)-\mathrm{B}(7)-\mathrm{B}(8)$ & $-143.99(15)$ \\
\hline $\mathrm{B}(3)-\mathrm{C}(2)-\mathrm{B}(7)-\mathrm{B}(8)$ & $-34.79(16)$ \\
\hline $\mathrm{C}(1)-\mathrm{B}(6)-\mathrm{B}(7)-\mathrm{C}(2)$ & $-34.40(13)$ \\
\hline $\mathrm{B}(5)-\mathrm{B}(6)-\mathrm{B}(7)-\mathrm{C}(2)$ & $-95.64(16)$ \\
\hline $\mathrm{B}(11)-\mathrm{B}(6)-\mathrm{B}(7)-\mathrm{C}(2)$ & $-133.39(16)$ \\
\hline $\mathrm{C}(2)-\mathrm{B}(6)-\mathrm{B}(7)-\mathrm{B}(11)$ & $133.39(16)$ \\
\hline $\mathrm{C}(1)-\mathrm{B}(6)-\mathrm{B}(7)-\mathrm{B}(11)$ & $98.99(16)$ \\
\hline $\mathrm{B}(5)-\mathrm{B}(6)-\mathrm{B}(7)-\mathrm{B}(11)$ & $37.75(15)$ \\
\hline $\mathrm{C}(2)-\mathrm{B}(6)-\mathrm{B}(7)-\mathrm{B}(12)$ & $95.11(16)$ \\
\hline $\mathrm{C}(1)-\mathrm{B}(6)-\mathrm{B}(7)-\mathrm{B}(12)$ & $60.71(18)$ \\
\hline $\mathrm{B}(5)-\mathrm{B}(6)-\mathrm{B}(7)-\mathrm{B}(12)$ & $-0.5(2)$ \\
\hline $\mathrm{B}(11)-\mathrm{B}(6)-\mathrm{B}(7)-\mathrm{B}(12)$ & $-38.28(15)$ \\
\hline $\mathrm{C}(2)-\mathrm{B}(6)-\mathrm{B}(7)-\mathrm{B}(8)$ & $31.67(13)$ \\
\hline
\end{tabular}




\begin{tabular}{|c|c|}
\hline $\mathrm{C}(1)-\mathrm{B}(6)-\mathrm{B}(7)-\mathrm{B}(8)$ & $-2.73(19)$ \\
\hline $\mathrm{B}(5)-\mathrm{B}(6)-\mathrm{B}(7)-\mathrm{B}(8)$ & $-63.97(19)$ \\
\hline $\mathrm{B}(11)-\mathrm{B}(6)-\mathrm{B}(7)-\mathrm{B}(8)$ & $-101.72(16)$ \\
\hline $\mathrm{C}(1)-\mathrm{C}(2)-\mathrm{B}(8)-\mathrm{B}(9)$ & $3.2(2)$ \\
\hline $\mathrm{B}(7)-\mathrm{C}(2)-\mathrm{B}(8)-\mathrm{B}(9)$ & $-102.33(16)$ \\
\hline $\mathrm{B}(6)-\mathrm{C}(2)-\mathrm{B}(8)-\mathrm{B}(9)$ & $-66.0(2)$ \\
\hline $\mathrm{B}(3)-\mathrm{C}(2)-\mathrm{B}(8)-\mathrm{B}(9)$ & $43.07(14)$ \\
\hline $\mathrm{C}(1)-\mathrm{C}(2)-\mathrm{B}(8)-\mathrm{B}(12)$ & $65.47(18)$ \\
\hline $\mathrm{B}(7)-\mathrm{C}(2)-\mathrm{B}(8)-\mathrm{B}(12)$ & $-40.02(14)$ \\
\hline $\mathrm{B}(6)-\mathrm{C}(2)-\mathrm{B}(8)-\mathrm{B}(12)$ & $-3.7(2)$ \\
\hline $\mathrm{B}(3)-\mathrm{C}(2)-\mathrm{B}(8)-\mathrm{B}(12)$ & $105.39(16)$ \\
\hline $\mathrm{C}(1)-\mathrm{C}(2)-\mathrm{B}(8)-\mathrm{B}(3)$ & $-39.91(15)$ \\
\hline $\mathrm{B}(7)-\mathrm{C}(2)-\mathrm{B}(8)-\mathrm{B}(3)$ & $-145.40(16)$ \\
\hline $\mathrm{B}(6)-\mathrm{C}(2)-\mathrm{B}(8)-\mathrm{B}(3)$ & $-109.05(17)$ \\
\hline $\mathrm{C}(1)-\mathrm{C}(2)-\mathrm{B}(8)-\mathrm{B}(7)$ & $105.49(17)$ \\
\hline $\mathrm{B}(6)-\mathrm{C}(2)-\mathrm{B}(8)-\mathrm{B}(7)$ & $36.35(16)$ \\
\hline $\mathrm{B}(3)-\mathrm{C}(2)-\mathrm{B}(8)-\mathrm{B}(7)$ & $145.40(16)$ \\
\hline $\mathrm{C}(1)-\mathrm{B}(3)-\mathrm{B}(8)-\mathrm{C}(2)$ & $34.01(12)$ \\
\hline $\mathrm{B}(9)-\mathrm{B}(3)-\mathrm{B}(8)-\mathrm{C}(2)$ & $130.62(16)$ \\
\hline $\mathrm{B}(4)-\mathrm{B}(3)-\mathrm{B}(8)-\mathrm{C}(2)$ & $91.87(15)$ \\
\hline $\operatorname{Ir}(1)-\mathrm{B}(3)-\mathrm{B}(8)-\mathrm{C}(2)$ & $-91.71(19)$ \\
\hline $\mathrm{C}(2)-\mathrm{B}(3)-\mathrm{B}(8)-\mathrm{B}(9)$ & $-130.62(16)$ \\
\hline $\mathrm{C}(1)-\mathrm{B}(3)-\mathrm{B}(8)-\mathrm{B}(9)$ & $-96.60(15)$ \\
\hline $\mathrm{B}(4)-\mathrm{B}(3)-\mathrm{B}(8)-\mathrm{B}(9)$ & $-38.75(14)$ \\
\hline $\operatorname{Ir}(1)-\mathrm{B}(3)-\mathrm{B}(8)-\mathrm{B}(9)$ & $137.7(2)$ \\
\hline $\mathrm{C}(2)-\mathrm{B}(3)-\mathrm{B}(8)-\mathrm{B}(12)$ & $-94.17(16)$ \\
\hline $\mathrm{C}(1)-\mathrm{B}(3)-\mathrm{B}(8)-\mathrm{B}(12)$ & $-60.16(17)$ \\
\hline $\mathrm{B}(9)-\mathrm{B}(3)-\mathrm{B}(8)-\mathrm{B}(12)$ & $36.45(15)$ \\
\hline $\mathrm{B}(4)-\mathrm{B}(3)-\mathrm{B}(8)-\mathrm{B}(12)$ & $-2.30(19)$ \\
\hline $\operatorname{Ir}(1)-\mathrm{B}(3)-\mathrm{B}(8)-\mathrm{B}(12)$ & $174.12(15)$ \\
\hline $\mathrm{C}(2)-\mathrm{B}(3)-\mathrm{B}(8)-\mathrm{B}(7)$ & $-30.62(14)$ \\
\hline $\mathrm{C}(1)-\mathrm{B}(3)-\mathrm{B}(8)-\mathrm{B}(7)$ & $3.40(18)$ \\
\hline $\mathrm{B}(9)-\mathrm{B}(3)-\mathrm{B}(8)-\mathrm{B}(7)$ & $100.00(17)$ \\
\hline $\mathrm{B}(4)-\mathrm{B}(3)-\mathrm{B}(8)-\mathrm{B}(7)$ & $61.25(18)$ \\
\hline $\operatorname{Ir}(1)-\mathrm{B}(3)-\mathrm{B}(8)-\mathrm{B}(7)$ & $-122.33(18)$ \\
\hline $\mathrm{B}(11)-\mathrm{B}(7)-\mathrm{B}(8)-\mathrm{C}(2)$ & $-95.63(16)$ \\
\hline
\end{tabular}




\begin{tabular}{|c|c|}
\hline $\mathrm{B}(12)-\mathrm{B}(7)-\mathrm{B}(8)-\mathrm{C}(2)$ & $-133.34(16)$ \\
\hline $\mathrm{B}(6)-\mathrm{B}(7)-\mathrm{B}(8)-\mathrm{C}(2)$ & $-32.10(14)$ \\
\hline $\mathrm{C}(2)-\mathrm{B}(7)-\mathrm{B}(8)-\mathrm{B}(9)$ & $96.02(16)$ \\
\hline $\mathrm{B}(11)-\mathrm{B}(7)-\mathrm{B}(8)-\mathrm{B}(9)$ & $0.4(2)$ \\
\hline $\mathrm{B}(12)-\mathrm{B}(7)-\mathrm{B}(8)-\mathrm{B}(9)$ & $-37.32(15)$ \\
\hline $\mathrm{B}(6)-\mathrm{B}(7)-\mathrm{B}(8)-\mathrm{B}(9)$ & $63.92(18)$ \\
\hline $\mathrm{C}(2)-\mathrm{B}(7)-\mathrm{B}(8)-\mathrm{B}(12)$ & $133.34(16)$ \\
\hline $\mathrm{B}(11)-\mathrm{B}(7)-\mathrm{B}(8)-\mathrm{B}(12)$ & $37.71(15)$ \\
\hline $\mathrm{B}(6)-\mathrm{B}(7)-\mathrm{B}(8)-\mathrm{B}(12)$ & 101.24(17) \\
\hline $\mathrm{C}(2)-\mathrm{B}(7)-\mathrm{B}(8)-\mathrm{B}(3)$ & $31.60(14)$ \\
\hline $\mathrm{B}(11)-\mathrm{B}(7)-\mathrm{B}(8)-\mathrm{B}(3)$ & $-64.03(19)$ \\
\hline $\mathrm{B}(12)-\mathrm{B}(7)-\mathrm{B}(8)-\mathrm{B}(3)$ & $-101.74(17)$ \\
\hline $\mathrm{B}(6)-\mathrm{B}(7)-\mathrm{B}(8)-\mathrm{B}(3)$ & $-0.5(2)$ \\
\hline $\mathrm{C}(2)-\mathrm{B}(8)-\mathrm{B}(9)-\mathrm{B}(4)$ & $-2.2(2)$ \\
\hline $\mathrm{B}(12)-\mathrm{B}(8)-\mathrm{B}(9)-\mathrm{B}(4)$ & $-99.26(17)$ \\
\hline $\mathrm{B}(3)-\mathrm{B}(8)-\mathrm{B}(9)-\mathrm{B}(4)$ & $40.68(14)$ \\
\hline $\mathrm{B}(7)-\mathrm{B}(8)-\mathrm{B}(9)-\mathrm{B}(4)$ & $-62.33(19)$ \\
\hline $\mathrm{C}(2)-\mathrm{B}(8)-\mathrm{B}(9)-\mathrm{B}(12)$ & $97.03(16)$ \\
\hline $\mathrm{B}(3)-\mathrm{B}(8)-\mathrm{B}(9)-\mathrm{B}(12)$ & 139.93(16) \\
\hline $\mathrm{B}(7)-\mathrm{B}(8)-\mathrm{B}(9)-\mathrm{B}(12)$ & $36.92(15)$ \\
\hline $\mathrm{C}(2)-\mathrm{B}(8)-\mathrm{B}(9)-\mathrm{B}(10)$ & $59.96(18)$ \\
\hline $\mathrm{B}(12)-\mathrm{B}(8)-\mathrm{B}(9)-\mathrm{B}(10)$ & $-37.08(15)$ \\
\hline $\mathrm{B}(3)-\mathrm{B}(8)-\mathrm{B}(9)-\mathrm{B}(10)$ & $102.86(16)$ \\
\hline $\mathrm{B}(7)-\mathrm{B}(8)-\mathrm{B}(9)-\mathrm{B}(10)$ & $-0.2(2)$ \\
\hline $\mathrm{C}(2)-\mathrm{B}(8)-\mathrm{B}(9)-\mathrm{B}(3)$ & $-42.90(14)$ \\
\hline $\mathrm{B}(12)-\mathrm{B}(8)-\mathrm{B}(9)-\mathrm{B}(3)$ & $-139.93(16)$ \\
\hline $\mathrm{B}(7)-\mathrm{B}(8)-\mathrm{B}(9)-\mathrm{B}(3)$ & $-103.01(16)$ \\
\hline $\mathrm{C}(1)-\mathrm{B}(4)-\mathrm{B}(9)-\mathrm{B}(8)$ & $0.6(2)$ \\
\hline $\mathrm{B}(10)-\mathrm{B}(4)-\mathrm{B}(9)-\mathrm{B}(8)$ & $100.25(17)$ \\
\hline $\mathrm{B}(5)-\mathrm{B}(4)-\mathrm{B}(9)-\mathrm{B}(8)$ & $62.97(19)$ \\
\hline $\mathrm{B}(3)-\mathrm{B}(4)-\mathrm{B}(9)-\mathrm{B}(8)$ & $-40.24(14)$ \\
\hline $\mathrm{C}(1)-\mathrm{B}(4)-\mathrm{B}(9)-\mathrm{B}(12)$ & $-62.44(18)$ \\
\hline $\mathrm{B}(10)-\mathrm{B}(4)-\mathrm{B}(9)-\mathrm{B}(12)$ & $37.17(14)$ \\
\hline $\mathrm{B}(5)-\mathrm{B}(4)-\mathrm{B}(9)-\mathrm{B}(12)$ & $-0.12(19)$ \\
\hline $\mathrm{B}(3)-\mathrm{B}(4)-\mathrm{B}(9)-\mathrm{B}(12)$ & $-103.32(16)$ \\
\hline $\mathrm{C}(1)-\mathrm{B}(4)-\mathrm{B}(9)-\mathrm{B}(10)$ & $-99.61(16)$ \\
\hline
\end{tabular}


$\mathrm{B}(5)-\mathrm{B}(4)-\mathrm{B}(9)-\mathrm{B}(10)$

$-37.28(14)$

$\mathrm{B}(3)-\mathrm{B}(4)-\mathrm{B}(9)-\mathrm{B}(10)$

$-140.48(16)$

$\mathrm{C}(1)-\mathrm{B}(4)-\mathrm{B}(9)-\mathrm{B}(3)$

40.88(14)

$\mathrm{B}(10)-\mathrm{B}(4)-\mathrm{B}(9)-\mathrm{B}(3)$

$140.48(16)$

$\mathrm{B}(5)-\mathrm{B}(4)-\mathrm{B}(9)-\mathrm{B}(3)$

103.20(16)

$\mathrm{C}(2)-\mathrm{B}(3)-\mathrm{B}(9)-\mathrm{B}(8)$

40.30(14)

$\mathrm{C}(1)-\mathrm{B}(3)-\mathrm{B}(9)-\mathrm{B}(8)$

96.76(15)

$\mathrm{B}(4)-\mathrm{B}(3)-\mathrm{B}(9)-\mathrm{B}(8)$

134.80(16)

$\operatorname{Ir}(1)-\mathrm{B}(3)-\mathrm{B}(9)-\mathrm{B}(8)$

$-128.9(3)$

$\mathrm{C}(2)-\mathrm{B}(3)-\mathrm{B}(9)-\mathrm{B}(4)$

$-94.50(15)$

$\mathrm{C}(1)-\mathrm{B}(3)-\mathrm{B}(9)-\mathrm{B}(4)$

$-38.04(13)$

$\mathrm{B}(8)-\mathrm{B}(3)-\mathrm{B}(9)-\mathrm{B}(4)$

-134.80(16)

$\operatorname{Ir}(1)-B(3)-B(9)-B(4)$

96.3(2)

$\mathrm{C}(2)-\mathrm{B}(3)-\mathrm{B}(9)-\mathrm{B}(12)$

4.08(19)

$\mathrm{C}(1)-\mathrm{B}(3)-\mathrm{B}(9)-\mathrm{B}(12)$

60.54(18)

$\mathrm{B}(8)-\mathrm{B}(3)-\mathrm{B}(9)-\mathrm{B}(12)$

$-36.22(15)$

$\mathrm{B}(4)-\mathrm{B}(3)-\mathrm{B}(9)-\mathrm{B}(12)$

98.58(17)

$\operatorname{Ir}(1)-\mathrm{B}(3)-\mathrm{B}(9)-\mathrm{B}(12)$

$-165.10(19)$

$\mathrm{C}(2)-\mathrm{B}(3)-\mathrm{B}(9)-\mathrm{B}(10)$

$-59.15(18)$

$\mathrm{C}(1)-\mathrm{B}(3)-\mathrm{B}(9)-\mathrm{B}(10)$

$-2.69(19)$

$\mathrm{B}(8)-\mathrm{B}(3)-\mathrm{B}(9)-\mathrm{B}(10)$

$-99.45(17)$

$\mathrm{B}(4)-\mathrm{B}(3)-\mathrm{B}(9)-\mathrm{B}(10)$

$35.35(15)$

$\operatorname{Ir}(1)-\mathrm{B}(3)-\mathrm{B}(9)-\mathrm{B}(10)$

131.7(2)

$\mathrm{C}(1)-\mathrm{B}(4)-\mathrm{B}(10)-\mathrm{B}(12)$

61.76(19)

$\mathrm{B}(9)-\mathrm{B}(4)-\mathrm{B}(10)-\mathrm{B}(12)$

$-37.76(15)$

$\mathrm{B}(5)-\mathrm{B}(4)-\mathrm{B}(10)-\mathrm{B}(12)$

101.21(17)

$\mathrm{B}(3)-\mathrm{B}(4)-\mathrm{B}(10)-\mathrm{B}(12)$

$-1.8(2)$

$\mathrm{C}(1)-\mathrm{B}(4)-\mathrm{B}(10)-\mathrm{B}(5)$

$-39.45(14)$

$\mathrm{B}(9)-\mathrm{B}(4)-\mathrm{B}(10)-\mathrm{B}(5)$

$-138.97(16)$

$\mathrm{B}(3)-\mathrm{B}(4)-\mathrm{B}(10)-\mathrm{B}(5)$

$-102.99(16)$

$\mathrm{C}(1)-\mathrm{B}(4)-\mathrm{B}(10)-\mathrm{B}(11)$

$-2.2(2)$

$\mathrm{B}(9)-\mathrm{B}(4)-\mathrm{B}(10)-\mathrm{B}(11)$

$-101.70(17)$

$\mathrm{B}(5)-\mathrm{B}(4)-\mathrm{B}(10)-\mathrm{B}(11)$

37.27(15)

$\mathrm{B}(3)-\mathrm{B}(4)-\mathrm{B}(10)-\mathrm{B}(11)$

$-65.72(19)$

$\mathrm{C}(1)-\mathrm{B}(4)-\mathrm{B}(10)-\mathrm{B}(9)$

99.52(16)

$\mathrm{B}(5)-\mathrm{B}(4)-\mathrm{B}(10)-\mathrm{B}(9)$

138.97(16) 


\begin{tabular}{|c|c|}
\hline $\mathrm{B}(3)-\mathrm{B}(4)-\mathrm{B}(10)-\mathrm{B}(9)$ & $35.98(14)$ \\
\hline $\mathrm{C}(1)-\mathrm{B}(5)-\mathrm{B}(10)-\mathrm{B}(4)$ & $38.32(14)$ \\
\hline $\mathrm{B}(11)-\mathrm{B}(5)-\mathrm{B}(10)-\mathrm{B}(4)$ & $137.88(16)$ \\
\hline $\mathrm{B}(6)-\mathrm{B}(5)-\mathrm{B}(10)-\mathrm{B}(4)$ & $100.06(17)$ \\
\hline $\mathrm{C}(1)-\mathrm{B}(5)-\mathrm{B}(10)-\mathrm{B}(12)$ & $-62.04(19)$ \\
\hline $\mathrm{B}(11)-\mathrm{B}(5)-\mathrm{B}(10)-\mathrm{B}(12)$ & $37.52(15)$ \\
\hline $\mathrm{B}(6)-\mathrm{B}(5)-\mathrm{B}(10)-\mathrm{B}(12)$ & $-0.3(2)$ \\
\hline $\mathrm{B}(4)-\mathrm{B}(5)-\mathrm{B}(10)-\mathrm{B}(12)$ & $-100.36(17)$ \\
\hline $\mathrm{C}(1)-\mathrm{B}(5)-\mathrm{B}(10)-\mathrm{B}(11)$ & $-99.56(16)$ \\
\hline $\mathrm{B}(6)-\mathrm{B}(5)-\mathrm{B}(10)-\mathrm{B}(11)$ & $-37.82(15)$ \\
\hline $\mathrm{B}(4)-\mathrm{B}(5)-\mathrm{B}(10)-\mathrm{B}(11)$ & $-137.88(16)$ \\
\hline $\mathrm{C}(1)-\mathrm{B}(5)-\mathrm{B}(10)-\mathrm{B}(9)$ & $1.5(2)$ \\
\hline $\mathrm{B}(11)-\mathrm{B}(5)-\mathrm{B}(10)-\mathrm{B}(9)$ & $101.10(17)$ \\
\hline $\mathrm{B}(6)-\mathrm{B}(5)-\mathrm{B}(10)-\mathrm{B}(9)$ & $63.29(19)$ \\
\hline $\mathrm{B}(4)-\mathrm{B}(5)-\mathrm{B}(10)-\mathrm{B}(9)$ & $-36.78(15)$ \\
\hline $\mathrm{B}(8)-\mathrm{B}(9)-\mathrm{B}(10)-\mathrm{B}(4)$ & $-100.18(16)$ \\
\hline $\mathrm{B}(12)-\mathrm{B}(9)-\mathrm{B}(10)-\mathrm{B}(4)$ & $-137.61(16)$ \\
\hline $\mathrm{B}(3)-\mathrm{B}(9)-\mathrm{B}(10)-\mathrm{B}(4)$ & $-36.26(15)$ \\
\hline $\mathrm{B}(8)-\mathrm{B}(9)-\mathrm{B}(10)-\mathrm{B}(12)$ & $37.43(14)$ \\
\hline $\mathrm{B}(4)-\mathrm{B}(9)-\mathrm{B}(10)-\mathrm{B}(12)$ & 137.61(16) \\
\hline $\mathrm{B}(3)-\mathrm{B}(9)-\mathrm{B}(10)-\mathrm{B}(12)$ & $101.35(17)$ \\
\hline $\mathrm{B}(8)-\mathrm{B}(9)-\mathrm{B}(10)-\mathrm{B}(5)$ & $-63.12(19)$ \\
\hline $\mathrm{B}(4)-\mathrm{B}(9)-\mathrm{B}(10)-\mathrm{B}(5)$ & $37.06(15)$ \\
\hline $\mathrm{B}(12)-\mathrm{B}(9)-\mathrm{B}(10)-\mathrm{B}(5)$ & $-100.55(17)$ \\
\hline $\mathrm{B}(3)-\mathrm{B}(9)-\mathrm{B}(10)-\mathrm{B}(5)$ & $0.8(2)$ \\
\hline $\mathrm{B}(8)-\mathrm{B}(9)-\mathrm{B}(10)-\mathrm{B}(11)$ & $-0.1(2)$ \\
\hline $\mathrm{B}(4)-\mathrm{B}(9)-\mathrm{B}(10)-\mathrm{B}(11)$ & $100.06(16)$ \\
\hline $\mathrm{B}(12)-\mathrm{B}(9)-\mathrm{B}(10)-\mathrm{B}(11)$ & $-37.56(14)$ \\
\hline $\mathrm{B}(3)-\mathrm{B}(9)-\mathrm{B}(10)-\mathrm{B}(11)$ & $63.79(19)$ \\
\hline $\mathrm{C}(2)-\mathrm{B}(7)-\mathrm{B}(11)-\mathrm{B}(5)$ & $2.1(2)$ \\
\hline $\mathrm{B}(12)-\mathrm{B}(7)-\mathrm{B}(11)-\mathrm{B}(5)$ & $99.92(17)$ \\
\hline $\mathrm{B}(6)-\mathrm{B}(7)-\mathrm{B}(11)-\mathrm{B}(5)$ & $-37.94(15)$ \\
\hline $\mathrm{B}(8)-\mathrm{B}(7)-\mathrm{B}(11)-\mathrm{B}(5)$ & $62.76(19)$ \\
\hline $\mathrm{C}(2)-\mathrm{B}(7)-\mathrm{B}(11)-\mathrm{B}(6)$ & $40.09(14)$ \\
\hline $\mathrm{B}(12)-\mathrm{B}(7)-\mathrm{B}(11)-\mathrm{B}(6)$ & $137.86(16)$ \\
\hline $\mathrm{B}(8)-\mathrm{B}(7)-\mathrm{B}(11)-\mathrm{B}(6)$ & $100.70(16)$ \\
\hline
\end{tabular}




\begin{tabular}{|c|c|}
\hline $\mathrm{C}(2)-\mathrm{B}(7)-\mathrm{B}(11)-\mathrm{B}(10)$ & $-61.08(18)$ \\
\hline $\mathrm{B}(12)-\mathrm{B}(7)-\mathrm{B}(11)-\mathrm{B}(10)$ & $36.70(14)$ \\
\hline $\mathrm{B}(6)-\mathrm{B}(7)-\mathrm{B}(11)-\mathrm{B}(10)$ & $-101.17(16)$ \\
\hline $\mathrm{B}(8)-\mathrm{B}(7)-\mathrm{B}(11)-\mathrm{B}(10)$ & $-0.5(2)$ \\
\hline $\mathrm{C}(2)-\mathrm{B}(7)-\mathrm{B}(11)-\mathrm{B}(12)$ & $-97.78(16)$ \\
\hline $\mathrm{B}(6)-\mathrm{B}(7)-\mathrm{B}(11)-\mathrm{B}(12)$ & $-137.86(16)$ \\
\hline $\mathrm{B}(8)-\mathrm{B}(7)-\mathrm{B}(11)-\mathrm{B}(12)$ & $-37.16(14)$ \\
\hline $\mathrm{C}(1)-\mathrm{B}(5)-\mathrm{B}(11)-\mathrm{B}(7)$ & $-2.0(2)$ \\
\hline $\mathrm{B}(6)-\mathrm{B}(5)-\mathrm{B}(11)-\mathrm{B}(7)$ & $38.21(15)$ \\
\hline $\mathrm{B}(10)-\mathrm{B}(5)-\mathrm{B}(11)-\mathrm{B}(7)$ & $-99.61(17)$ \\
\hline $\mathrm{B}(4)-\mathrm{B}(5)-\mathrm{B}(11)-\mathrm{B}(7)$ & $-62.41(19)$ \\
\hline $\mathrm{C}(1)-\mathrm{B}(5)-\mathrm{B}(11)-\mathrm{B}(6)$ & $-40.25(14)$ \\
\hline $\mathrm{B}(10)-\mathrm{B}(5)-\mathrm{B}(11)-\mathrm{B}(6)$ & $-137.82(17)$ \\
\hline $\mathrm{B}(4)-\mathrm{B}(5)-\mathrm{B}(11)-\mathrm{B}(6)$ & $-100.62(16)$ \\
\hline $\mathrm{C}(1)-\mathrm{B}(5)-\mathrm{B}(11)-\mathrm{B}(10)$ & $97.57(16)$ \\
\hline $\mathrm{B}(6)-\mathrm{B}(5)-\mathrm{B}(11)-\mathrm{B}(10)$ & $137.82(17)$ \\
\hline $\mathrm{B}(4)-\mathrm{B}(5)-\mathrm{B}(11)-\mathrm{B}(10)$ & $37.20(14)$ \\
\hline $\mathrm{C}(1)-\mathrm{B}(5)-\mathrm{B}(11)-\mathrm{B}(12)$ & $60.88(18)$ \\
\hline $\mathrm{B}(6)-\mathrm{B}(5)-\mathrm{B}(11)-\mathrm{B}(12)$ & 101.13(16) \\
\hline $\mathrm{B}(10)-\mathrm{B}(5)-\mathrm{B}(11)-\mathrm{B}(12)$ & $-36.69(15)$ \\
\hline $\mathrm{B}(4)-\mathrm{B}(5)-\mathrm{B}(11)-\mathrm{B}(12)$ & $0.51(19)$ \\
\hline $\mathrm{C}(2)-\mathrm{B}(6)-\mathrm{B}(11)-\mathrm{B}(7)$ & $-38.98(14)$ \\
\hline $\mathrm{C}(1)-\mathrm{B}(6)-\mathrm{B}(11)-\mathrm{B}(7)$ & $-97.56(16)$ \\
\hline $\mathrm{B}(5)-\mathrm{B}(6)-\mathrm{B}(11)-\mathrm{B}(7)$ & $-137.54(16)$ \\
\hline $\mathrm{C}(2)-\mathrm{B}(6)-\mathrm{B}(11)-\mathrm{B}(5)$ & $98.56(16)$ \\
\hline $\mathrm{C}(1)-\mathrm{B}(6)-\mathrm{B}(11)-\mathrm{B}(5)$ & $39.98(14)$ \\
\hline $\mathrm{B}(7)-\mathrm{B}(6)-\mathrm{B}(11)-\mathrm{B}(5)$ & 137.54(16) \\
\hline $\mathrm{C}(2)-\mathrm{B}(6)-\mathrm{B}(11)-\mathrm{B}(10)$ & $60.93(19)$ \\
\hline $\mathrm{C}(1)-\mathrm{B}(6)-\mathrm{B}(11)-\mathrm{B}(10)$ & $2.4(2)$ \\
\hline $\mathrm{B}(5)-\mathrm{B}(6)-\mathrm{B}(11)-\mathrm{B}(10)$ & $-37.63(15)$ \\
\hline $\mathrm{B}(7)-\mathrm{B}(6)-\mathrm{B}(11)-\mathrm{B}(10)$ & 99.91(17) \\
\hline $\mathrm{C}(2)-\mathrm{B}(6)-\mathrm{B}(11)-\mathrm{B}(12)$ & $-1.49(19)$ \\
\hline $\mathrm{C}(1)-\mathrm{B}(6)-\mathrm{B}(11)-\mathrm{B}(12)$ & $-60.07(18)$ \\
\hline $\mathrm{B}(5)-\mathrm{B}(6)-\mathrm{B}(11)-\mathrm{B}(12)$ & $-100.05(17)$ \\
\hline $\mathrm{B}(7)-\mathrm{B}(6)-\mathrm{B}(11)-\mathrm{B}(12)$ & $37.49(15)$ \\
\hline $\mathrm{B}(4)-\mathrm{B}(10)-\mathrm{B}(11)-\mathrm{B}(7)$ & $63.65(19)$ \\
\hline
\end{tabular}




\begin{tabular}{|c|c|}
\hline $\mathrm{B}(12)-\mathrm{B}(10)-\mathrm{B}(11)-\mathrm{B}(7)$ & $-36.93(14)$ \\
\hline $\mathrm{B}(5)-\mathrm{B}(10)-\mathrm{B}(11)-\mathrm{B}(7)$ & $101.42(16)$ \\
\hline $\mathrm{B}(9)-\mathrm{B}(10)-\mathrm{B}(11)-\mathrm{B}(7)$ & $0.4(2)$ \\
\hline $\mathrm{B}(4)-\mathrm{B}(10)-\mathrm{B}(11)-\mathrm{B}(5)$ & $-37.77(15)$ \\
\hline $\mathrm{B}(12)-\mathrm{B}(10)-\mathrm{B}(11)-\mathrm{B}(5)$ & $-138.35(16)$ \\
\hline $\mathrm{B}(9)-\mathrm{B}(10)-\mathrm{B}(11)-\mathrm{B}(5)$ & $-101.05(17)$ \\
\hline $\mathrm{B}(4)-\mathrm{B}(10)-\mathrm{B}(11)-\mathrm{B}(6)$ & $-0.1(2)$ \\
\hline $\mathrm{B}(12)-\mathrm{B}(10)-\mathrm{B}(11)-\mathrm{B}(6)$ & $-100.73(17)$ \\
\hline $\mathrm{B}(5)-\mathrm{B}(10)-\mathrm{B}(11)-\mathrm{B}(6)$ & $37.62(15)$ \\
\hline $\mathrm{B}(9)-\mathrm{B}(10)-\mathrm{B}(11)-\mathrm{B}(6)$ & $-63.4(2)$ \\
\hline $\mathrm{B}(4)-\mathrm{B}(10)-\mathrm{B}(11)-\mathrm{B}(12)$ & $100.58(17)$ \\
\hline $\mathrm{B}(5)-\mathrm{B}(10)-\mathrm{B}(11)-\mathrm{B}(12)$ & $138.35(16)$ \\
\hline $\mathrm{B}(9)-\mathrm{B}(10)-\mathrm{B}(11)-\mathrm{B}(12)$ & $37.30(15)$ \\
\hline $\mathrm{B}(4)-\mathrm{B}(10)-\mathrm{B}(12)-\mathrm{B}(7)$ & $-63.9(2)$ \\
\hline $\mathrm{B}(5)-\mathrm{B}(10)-\mathrm{B}(12)-\mathrm{B}(7)$ & $0.0(2)$ \\
\hline $\mathrm{B}(11)-\mathrm{B}(10)-\mathrm{B}(12)-\mathrm{B}(7)$ & $36.93(15)$ \\
\hline $\mathrm{B}(9)-\mathrm{B}(10)-\mathrm{B}(12)-\mathrm{B}(7)$ & $-101.55(17)$ \\
\hline $\mathrm{B}(4)-\mathrm{B}(10)-\mathrm{B}(12)-\mathrm{B}(8)$ & $0.3(2)$ \\
\hline $\mathrm{B}(5)-\mathrm{B}(10)-\mathrm{B}(12)-\mathrm{B}(8)$ & $64.1(2)$ \\
\hline $\mathrm{B}(11)-\mathrm{B}(10)-\mathrm{B}(12)-\mathrm{B}(8)$ & 101.11(16) \\
\hline $\mathrm{B}(9)-\mathrm{B}(10)-\mathrm{B}(12)-\mathrm{B}(8)$ & $-37.37(14)$ \\
\hline $\mathrm{B}(4)-\mathrm{B}(10)-\mathrm{B}(12)-\mathrm{B}(9)$ & $37.67(15)$ \\
\hline $\mathrm{B}(5)-\mathrm{B}(10)-\mathrm{B}(12)-\mathrm{B}(9)$ & $101.52(17)$ \\
\hline $\mathrm{B}(11)-\mathrm{B}(10)-\mathrm{B}(12)-\mathrm{B}(9)$ & $138.48(15)$ \\
\hline $\mathrm{B}(4)-\mathrm{B}(10)-\mathrm{B}(12)-\mathrm{B}(11)$ & $-100.81(16)$ \\
\hline $\mathrm{B}(5)-\mathrm{B}(10)-\mathrm{B}(12)-\mathrm{B}(11)$ & $-36.97(15)$ \\
\hline $\mathrm{B}(9)-\mathrm{B}(10)-\mathrm{B}(12)-\mathrm{B}(11)$ & $-138.48(15)$ \\
\hline $\mathrm{C}(2)-\mathrm{B}(7)-\mathrm{B}(12)-\mathrm{B}(10)$ & $61.89(19)$ \\
\hline $\mathrm{B}(11)-\mathrm{B}(7)-\mathrm{B}(12)-\mathrm{B}(10)$ & $-37.37(15)$ \\
\hline $\mathrm{B}(6)-\mathrm{B}(7)-\mathrm{B}(12)-\mathrm{B}(10)$ & $0.4(2)$ \\
\hline $\mathrm{B}(8)-\mathrm{B}(7)-\mathrm{B}(12)-\mathrm{B}(10)$ & $101.26(17)$ \\
\hline $\mathrm{C}(2)-\mathrm{B}(7)-\mathrm{B}(12)-\mathrm{B}(8)$ & $-39.37(14)$ \\
\hline $\mathrm{B}(11)-\mathrm{B}(7)-\mathrm{B}(12)-\mathrm{B}(8)$ & $-138.64(16)$ \\
\hline $\mathrm{B}(6)-\mathrm{B}(7)-\mathrm{B}(12)-\mathrm{B}(8)$ & $-100.91(17)$ \\
\hline $\mathrm{C}(2)-\mathrm{B}(7)-\mathrm{B}(12)-\mathrm{B}(9)$ & $-2.3(2)$ \\
\hline $\mathrm{B}(11)-\mathrm{B}(7)-\mathrm{B}(12)-\mathrm{B}(9)$ & $-101.56(17)$ \\
\hline
\end{tabular}




\begin{tabular}{|c|c|}
\hline $\mathrm{B}(6)-\mathrm{B}(7)-\mathrm{B}(12)-\mathrm{B}(9)$ & $-63.8(2)$ \\
\hline $\mathrm{B}(8)-\mathrm{B}(7)-\mathrm{B}(12)-\mathrm{B}(9)$ & $37.08(15)$ \\
\hline $\mathrm{C}(2)-\mathrm{B}(7)-\mathrm{B}(12)-\mathrm{B}(11)$ & $99.26(16)$ \\
\hline $\mathrm{B}(6)-\mathrm{B}(7)-\mathrm{B}(12)-\mathrm{B}(11)$ & $37.72(15)$ \\
\hline $\mathrm{B}(8)-\mathrm{B}(7)-\mathrm{B}(12)-\mathrm{B}(11)$ & 138.64(16) \\
\hline $\mathrm{C}(2)-\mathrm{B}(8)-\mathrm{B}(12)-\mathrm{B}(10)$ & $-61.47(18)$ \\
\hline $\mathrm{B}(9)-\mathrm{B}(8)-\mathrm{B}(12)-\mathrm{B}(10)$ & $37.87(15)$ \\
\hline $\mathrm{B}(3)-\mathrm{B}(8)-\mathrm{B}(12)-\mathrm{B}(10)$ & $1.3(2)$ \\
\hline $\mathrm{B}(7)-\mathrm{B}(8)-\mathrm{B}(12)-\mathrm{B}(10)$ & $-100.55(17)$ \\
\hline $\mathrm{C}(2)-\mathrm{B}(8)-\mathrm{B}(12)-\mathrm{B}(7)$ & $39.08(14)$ \\
\hline $\mathrm{B}(9)-\mathrm{B}(8)-\mathrm{B}(12)-\mathrm{B}(7)$ & $138.42(16)$ \\
\hline $\mathrm{B}(3)-\mathrm{B}(8)-\mathrm{B}(12)-\mathrm{B}(7)$ & $101.86(16)$ \\
\hline $\mathrm{C}(2)-\mathrm{B}(8)-\mathrm{B}(12)-\mathrm{B}(9)$ & $-99.34(16)$ \\
\hline $\mathrm{B}(3)-\mathrm{B}(8)-\mathrm{B}(12)-\mathrm{B}(9)$ & $-36.56(15)$ \\
\hline $\mathrm{B}(7)-\mathrm{B}(8)-\mathrm{B}(12)-\mathrm{B}(9)$ & $-138.42(16)$ \\
\hline $\mathrm{C}(2)-\mathrm{B}(8)-\mathrm{B}(12)-\mathrm{B}(11)$ & $2.34(19)$ \\
\hline $\mathrm{B}(9)-\mathrm{B}(8)-\mathrm{B}(12)-\mathrm{B}(11)$ & $101.68(17)$ \\
\hline $\mathrm{B}(3)-\mathrm{B}(8)-\mathrm{B}(12)-\mathrm{B}(11)$ & $65.12(19)$ \\
\hline $\mathrm{B}(7)-\mathrm{B}(8)-\mathrm{B}(12)-\mathrm{B}(11)$ & $-36.74(15)$ \\
\hline $\mathrm{B}(8)-\mathrm{B}(9)-\mathrm{B}(12)-\mathrm{B}(10)$ & $-138.00(16)$ \\
\hline $\mathrm{B}(4)-\mathrm{B}(9)-\mathrm{B}(12)-\mathrm{B}(10)$ & $-37.05(14)$ \\
\hline $\mathrm{B}(3)-\mathrm{B}(9)-\mathrm{B}(12)-\mathrm{B}(10)$ & $-101.68(17)$ \\
\hline $\mathrm{B}(8)-\mathrm{B}(9)-\mathrm{B}(12)-\mathrm{B}(7)$ & $-37.59(15)$ \\
\hline $\mathrm{B}(4)-\mathrm{B}(9)-\mathrm{B}(12)-\mathrm{B}(7)$ & $63.36(19)$ \\
\hline $\mathrm{B}(10)-\mathrm{B}(9)-\mathrm{B}(12)-\mathrm{B}(7)$ & $100.41(17)$ \\
\hline $\mathrm{B}(3)-\mathrm{B}(9)-\mathrm{B}(12)-\mathrm{B}(7)$ & $-1.3(2)$ \\
\hline $\mathrm{B}(4)-\mathrm{B}(9)-\mathrm{B}(12)-\mathrm{B}(8)$ & $100.95(16)$ \\
\hline $\mathrm{B}(10)-\mathrm{B}(9)-\mathrm{B}(12)-\mathrm{B}(8)$ & $138.00(16)$ \\
\hline $\mathrm{B}(3)-\mathrm{B}(9)-\mathrm{B}(12)-\mathrm{B}(8)$ & $36.32(15)$ \\
\hline $\mathrm{B}(8)-\mathrm{B}(9)-\mathrm{B}(12)-\mathrm{B}(11)$ & $-100.52(16)$ \\
\hline $\mathrm{B}(4)-\mathrm{B}(9)-\mathrm{B}(12)-\mathrm{B}(11)$ & $0.4(2)$ \\
\hline $\mathrm{B}(10)-\mathrm{B}(9)-\mathrm{B}(12)-\mathrm{B}(11)$ & $37.49(14)$ \\
\hline $\mathrm{B}(3)-\mathrm{B}(9)-\mathrm{B}(12)-\mathrm{B}(11)$ & $-64.2(2)$ \\
\hline $\mathrm{B}(7)-\mathrm{B}(11)-\mathrm{B}(12)-\mathrm{B}(10)$ & $138.25(16)$ \\
\hline $\mathrm{B}(5)-\mathrm{B}(11)-\mathrm{B}(12)-\mathrm{B}(10)$ & $37.08(14)$ \\
\hline $\mathrm{B}(6)-\mathrm{B}(11)-\mathrm{B}(12)-\mathrm{B}(10)$ & $100.35(17)$ \\
\hline
\end{tabular}




\begin{tabular}{|c|c|}
\hline $\mathrm{B}(5)-\mathrm{B}(11)-\mathrm{B}(12)-\mathrm{B}(7)$ & $-101.17(16)$ \\
\hline $\mathrm{B}(6)-\mathrm{B}(11)-\mathrm{B}(12)-\mathrm{B}(7)$ & $-37.90(15)$ \\
\hline $\mathrm{B}(10)-\mathrm{B}(11)-\mathrm{B}(12)-\mathrm{B}(7)$ & $-138.25(16)$ \\
\hline $\mathrm{B}(7)-\mathrm{B}(11)-\mathrm{B}(12)-\mathrm{B}(8)$ & $37.37(15)$ \\
\hline $\mathrm{B}(5)-\mathrm{B}(11)-\mathrm{B}(12)-\mathrm{B}(8)$ & $-63.80(19)$ \\
\hline $\mathrm{B}(6)-\mathrm{B}(11)-\mathrm{B}(12)-\mathrm{B}(8)$ & $-0.5(2)$ \\
\hline $\mathrm{B}(10)-\mathrm{B}(11)-\mathrm{B}(12)-\mathrm{B}(8)$ & $-100.87(17)$ \\
\hline $\mathrm{B}(7)-\mathrm{B}(11)-\mathrm{B}(12)-\mathrm{B}(9)$ & $100.58(17)$ \\
\hline $\mathrm{B}(5)-\mathrm{B}(11)-\mathrm{B}(12)-\mathrm{B}(9)$ & $-0.6(2)$ \\
\hline $\mathrm{B}(6)-\mathrm{B}(11)-\mathrm{B}(12)-\mathrm{B}(9)$ & $62.68(19)$ \\
\hline $\mathrm{B}(10)-\mathrm{B}(11)-\mathrm{B}(12)-\mathrm{B}(9)$ & $-37.67(15)$ \\
\hline $\mathrm{C}(2)-\mathrm{C}(1)-\mathrm{P}(1)-\mathrm{C}(4)$ & $63.90(14)$ \\
\hline $\mathrm{B}(4)-\mathrm{C}(1)-\mathrm{P}(1)-\mathrm{C}(4)$ & $-173.88(13)$ \\
\hline $\mathrm{B}(5)-\mathrm{C}(1)-\mathrm{P}(1)-\mathrm{C}(4)$ & $-99.8(2)$ \\
\hline $\mathrm{B}(6)-\mathrm{C}(1)-\mathrm{P}(1)-\mathrm{C}(4)$ & $-4.2(2)$ \\
\hline $\mathrm{B}(3)-\mathrm{C}(1)-\mathrm{P}(1)-\mathrm{C}(4)$ & $124.43(11)$ \\
\hline $\mathrm{C}(2)-\mathrm{C}(1)-\mathrm{P}(1)-\mathrm{C}(3)$ & $-177.09(12)$ \\
\hline $\mathrm{B}(4)-\mathrm{C}(1)-\mathrm{P}(1)-\mathrm{C}(3)$ & $-54.87(15)$ \\
\hline $\mathrm{B}(5)-\mathrm{C}(1)-\mathrm{P}(1)-\mathrm{C}(3)$ & $19.2(2)$ \\
\hline $\mathrm{B}(6)-\mathrm{C}(1)-\mathrm{P}(1)-\mathrm{C}(3)$ & $114.85(18)$ \\
\hline $\mathrm{B}(3)-\mathrm{C}(1)-\mathrm{P}(1)-\mathrm{C}(3)$ & $-116.56(11)$ \\
\hline $\mathrm{C}(2)-\mathrm{C}(1)-\mathrm{P}(1)-\operatorname{Ir}(1)$ & $-60.02(11)$ \\
\hline $\mathrm{B}(4)-\mathrm{C}(1)-\mathrm{P}(1)-\operatorname{Ir}(1)$ & $62.20(13)$ \\
\hline B(5)-C(1)-P(1)-Ir(1) & $136.3(2)$ \\
\hline $\mathrm{B}(6)-\mathrm{C}(1)-\mathrm{P}(1)-\operatorname{Ir}(1)$ & $-128.08(17)$ \\
\hline B(3)-C(1)-P(1)-Ir(1) & $0.51(9)$ \\
\hline $\mathrm{C}(4)-\mathrm{P}(1)-\mathrm{C}(3)-\mathrm{C}(6)$ & $75.75(17)$ \\
\hline $\mathrm{C}(1)-\mathrm{P}(1)-\mathrm{C}(3)-\mathrm{C}(6)$ & $-39.59(17)$ \\
\hline $\operatorname{Ir}(1)-\mathrm{P}(1)-\mathrm{C}(3)-\mathrm{C}(6)$ & $-141.38(13)$ \\
\hline $\mathrm{C}(4)-\mathrm{P}(1)-\mathrm{C}(3)-\mathrm{C}(5)$ & $-51.86(17)$ \\
\hline $\mathrm{C}(1)-\mathrm{P}(1)-\mathrm{C}(3)-\mathrm{C}(5)$ & $-167.21(14)$ \\
\hline $\operatorname{Ir}(1)-\mathrm{P}(1)-\mathrm{C}(3)-\mathrm{C}(5)$ & $91.01(14)$ \\
\hline $\mathrm{C}(3)-\mathrm{P}(1)-\mathrm{C}(4)-\mathrm{C}(8)$ & $81.99(16)$ \\
\hline $\mathrm{C}(1)-\mathrm{P}(1)-\mathrm{C}(4)-\mathrm{C}(8)$ & $-161.07(14)$ \\
\hline $\operatorname{Ir}(1)-\mathrm{P}(1)-\mathrm{C}(4)-\mathrm{C}(8)$ & $-57.90(15)$ \\
\hline $\mathrm{C}(3)-\mathrm{P}(1)-\mathrm{C}(4)-\mathrm{C}(7)$ & $-46.71(17)$ \\
\hline
\end{tabular}


$\mathrm{C}(1)-\mathrm{P}(1)-\mathrm{C}(4)-\mathrm{C}(7)$

$70.22(16)$

$\operatorname{Ir}(1)-\mathrm{P}(1)-\mathrm{C}(4)-\mathrm{C}(7)$

$173.39(12)$

Symmetry transformations used to generate equivalent atoms: 


\section{X-Ray Structure Determination for Complex 7}

A yellow prism fragment $\left(0.516 \times 0.193 \times 0.068 \mathrm{~mm}^{3}\right)$ was used for the single crystal $\mathrm{x}$-ray diffraction study of $\left[\left[\mathrm{CB}_{11} \mathrm{H}_{11}\right] \mathrm{P}\left[\mathrm{C}_{3} \mathrm{H}_{7}\right]_{2} \mathrm{C}_{8} \mathrm{H}_{12} \mathrm{Ir}\right]^{-}$.N $\left[\mathrm{C}_{4} \mathrm{H}_{12}\right]^{+}$. $\left[\mathrm{C}_{4} \mathrm{H}_{8} \mathrm{O}\right]$ (sample vL147JE_0m). The crystal was coated with paratone oil and mounted on to a cryo-loop glass fiber. X-ray intensity data were collected at $100(2) \mathrm{K}$ on a Bruker APEX2 ${ }^{3}$ platform-CCD X-ray diffractometer system (fine focus Mo-radiation, $\lambda=0.71073 \AA$ A , 50KV/30mA power). The CCD detector was placed at a distance of $5.0600 \mathrm{~cm}$ from the crystal.

A total of 4800 frames were collected for a sphere of reflections (with scan width of $0.5^{\circ}$ in $\omega$ and $\phi$, starting $\omega$ and $2 \theta$ angles of $-30^{\circ}$, and $\phi$ angles of $0^{\circ}, 90^{\circ}, 120^{\circ}, 180^{\circ}, 240^{\circ}$, and $270^{\circ}$, for every 360 frames, and 720 frames with $\phi$-scan from $0-360^{\circ}, 10 \mathrm{sec} /$ frame exposure time). The frames were integrated using the Bruker SAINT software package ${ }^{4}$ and using a narrow-frame integration algorithm. Based on a monoclinic crystal system, the integrated frames yielded a total of 49431 reflections at a maximum $2 \theta$ angle of $61.012^{\circ}(0.70 \AA$ resolution $)$, of which 10323 were independent reflections $\left(\mathrm{R}_{\mathrm{int}}=0.0221, \mathrm{R}_{\mathrm{sig}}=0.0174\right.$, redundancy $=4.8$, completeness $\left.=100 \%\right)$ and $9978(96.7 \%)$ reflections were greater than $2 \sigma(\mathrm{I})$. The unit cell parameters were, $\mathbf{a}=$ 10.3713(3) $\mathrm{A}, \mathbf{b}=11.0485(3) \AA, \mathbf{c}=14.7843(4) \AA, \boldsymbol{\beta}=92.3520(5)^{\mathrm{o}}, \mathrm{V}=1692.67(8) \AA^{3}, \mathrm{Z}=2$, calculated density $\mathrm{D}_{\mathrm{c}}=1.454 \mathrm{~g} / \mathrm{cm}^{3}$. Absorption corrections were applied (absorption coefficient $\mu=4.090 \mathrm{~mm}^{-1} ; \min / \max$ transmission $\left.=0.227 / 0.768\right)$ to the raw intensity data using the SADABS program ${ }^{5}$.

The Bruker SHELXTL software package ${ }^{6}$ was used for phase determination and structure refinement. The distribution of intensities $\left(E^{2}-1=0.656\right)$ and systematic absent reflections indicated two possible space groups, P2(1) and P2(1)/m. The space group P2(1) (\#4) was later determined to be correct. Direct methods of phase determination followed by two Fourier cycles of refinement led to an electron density map from which most of the non-hydrogen atoms were identified in the asymmetric unit of the unit cell. With subsequent isotropic refinement, all of the non-hydrogen atoms were identified. There was one anion of $\left.\left[\mathrm{CB}_{11} \mathrm{H}_{11}\right] \mathrm{P}\left[\mathrm{C}_{3} \mathrm{H}_{7}\right]_{2} \mathrm{C}_{8} \mathrm{H}_{12} \mathrm{Ir}\right]^{-}$, one cation of $\mathrm{N}\left[\mathrm{C}_{4} \mathrm{H}_{12}\right]^{+}$, and one disordered THF solvent (disordered site occupancy ratios was $65 \% / 35 \%$ ) present in the asymmetric unit of the unit cell. 
Atomic coordinates, isotropic and anisotropic displacement parameters of all the non-hydrogen atoms were refined by means of a full matrix least-squares procedure on $\mathrm{F}^{2}$. The $\mathrm{H}$-atoms were included in the refinement in calculated positions riding on the atoms to which they were attached, except the $\mathrm{H}$-atoms bonded to $\mathrm{C} 1 \mathrm{~A}, \mathrm{C} 2 \mathrm{~A}, \mathrm{C} 5 \mathrm{~A}$, and $\mathrm{C} 6 \mathrm{~A}$ were refined unrestrained. The refinement converged at $\mathrm{R} 1=0.0201, \mathrm{wR} 2=0.0496$, with intensity $\mathrm{I}>2 \sigma(\mathrm{I})$. The absolute structure Flack parameter, $\mathrm{x}=-0.011(5)$. The largest peak/hole in the final difference map was $1.009 /-0.738 \mathrm{e} / \AA^{3}$. The high residual electron density peak/hole is probably due to absorption correction errors and unresolved possible THF disorder.

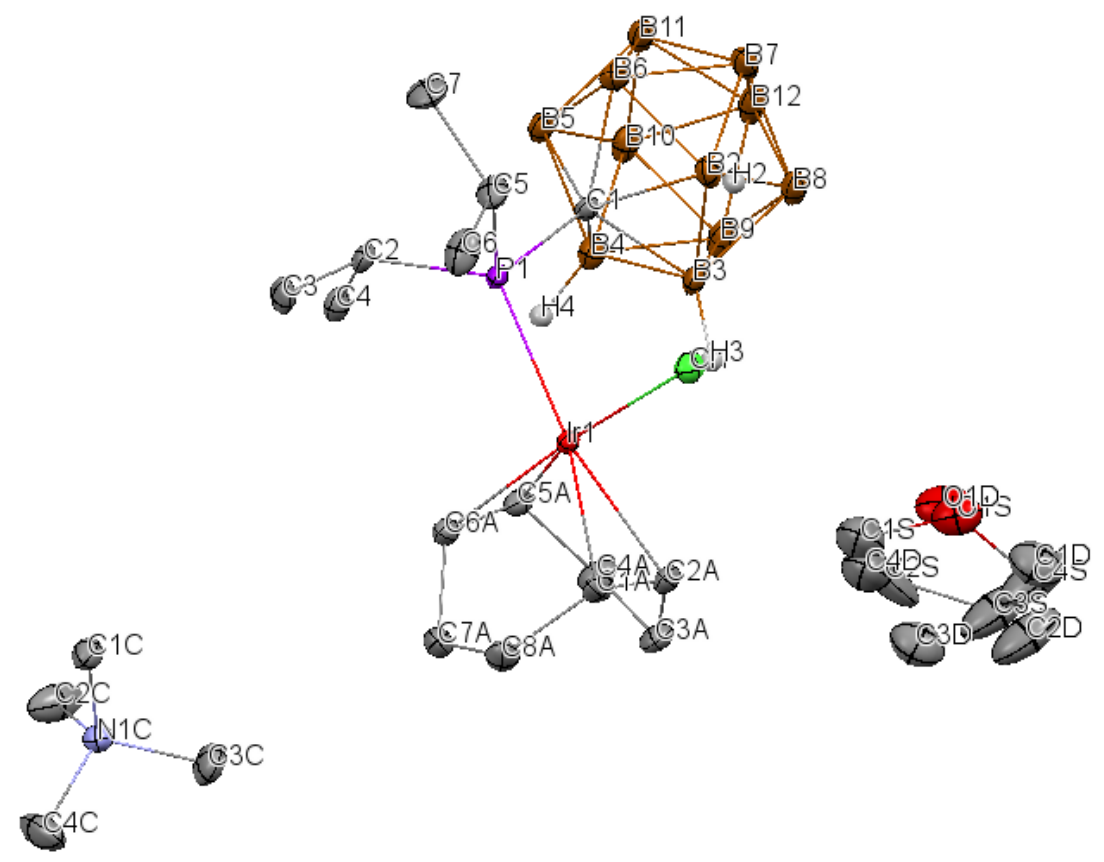

Full crystallographic data for compound $\mathbf{7}$ can be found at the Cambridge crystallographic database under reference \# CCDC 1057297 
Table 7. Crystal data and structure refinement for vL147JE_0m.

Identification code

Empirical formula

Formula weight

Temperature

Wavelength

Crystal system

Space group

Unit cell dimensions

Volume

$\mathrm{Z}$

Density (calculated)

Absorption coefficient

$\mathrm{F}(000)$

Crystal size

Theta range for data collection

Index ranges

Reflections collected

Independent reflections

Completeness to theta $=25.242^{\circ}$

Absorption correction

Refinement method

Data / restraints / parameters

Goodness-of-fit on $\mathrm{F}^{2}$

Final R indices [I $>2 \operatorname{sigma}(\mathrm{I})]$

$\mathrm{R}$ indices (all data)

Absolute structure parameter

Extinction coefficient

Largest diff. peak and hole
vL147JE_0m

C23 H57 B11 Cl Ir N O P

741.22

100(2) K

$0.71073 \AA$

Monoclinic

P 21

$\mathrm{a}=10.3713(3) \AA \quad \alpha=90^{\circ}$.

$\mathrm{b}=11.0485(3) \AA$ $\beta=92.3520(5)^{\circ}$.

$\mathrm{c}=14.7843(4) \AA$ $\gamma=90^{\circ}$.
2

$1.454 \mathrm{Mg} / \mathrm{m}^{3}$

$4.090 \mathrm{~mm}^{-1}$

748

$0.516 \times 0.193 \times 0.068 \mathrm{~mm}^{3}$

1.965 to $30.506^{\circ}$.

$-14<=\mathrm{h}<=14,-15<=\mathrm{k}<=15,-21<=\mathrm{l}<=21$

49431

$10323[\mathrm{R}(\mathrm{int})=0.0221]$

$100.0 \%$

Semi-empirical from equivalents

Full-matrix least-squares on $\mathrm{F}^{2}$

10323 / 52 / 394

1.056

$\mathrm{R} 1=0.0201, \mathrm{wR} 2=0.0496$

$\mathrm{R} 1=0.0211, \mathrm{wR} 2=0.0501$

$-0.011(5)$

$\mathrm{n} / \mathrm{a}$

1.009 and -0.738 e. $\AA^{-3}$ 
Table 8. Atomic coordinates ( $\left.\mathrm{x} 10^{4}\right)$ and equivalent isotropic displacement parameters $\left(\AA^{2} \mathrm{x}\right.$ $10^{3}$ )

for vL147JE_0m. U(eq) is defined as one third of the trace of the orthogonalized Uij tensor.

\begin{tabular}{|c|c|c|c|c|}
\hline & $\mathrm{x}$ & $\mathrm{y}$ & $\mathrm{z}$ & $\mathrm{U}(\mathrm{eq})$ \\
\hline \multicolumn{5}{|l|}{-} \\
\hline $\operatorname{Ir}(1)$ & $5454(1)$ & 2783(1) & $3336(1)$ & $10(1)$ \\
\hline $\mathrm{Cl}(1)$ & $5314(1)$ & $1935(1)$ & $1846(1)$ & $17(1)$ \\
\hline $\mathrm{C}(1 \mathrm{~A})$ & $3458(4)$ & 2196(4) & $3462(3)$ & $17(1)$ \\
\hline $\mathrm{C}(2 \mathrm{~A})$ & $3443(4)$ & $3381(4)$ & $3123(3)$ & $16(1)$ \\
\hline $\mathrm{C}(3 \mathrm{~A})$ & $3136(3)$ & $4488(4)$ & $3680(3)$ & $19(1)$ \\
\hline $\mathrm{C}(4 \mathrm{~A})$ & $4368(4)$ & $5077(3)$ & $4100(3)$ & $20(1)$ \\
\hline $\mathrm{C}(5 \mathrm{~A})$ & $5436(3)$ & $4171(3)$ & 4299(2) & $14(1)$ \\
\hline $\mathrm{C}(6 \mathrm{~A})$ & $5264(3)$ & $3051(3)$ & $4752(2)$ & $16(1)$ \\
\hline $\mathrm{C}(7 \mathrm{~A})$ & $3964(3)$ & $2662(6)$ & $5113(2)$ & $19(1)$ \\
\hline $\mathrm{C}(8 \mathrm{~A})$ & $3210(4)$ & $1866(4)$ & $4428(3)$ & $21(1)$ \\
\hline $\mathrm{P}(1)$ & $7741(1)$ & $2750(2)$ & $3351(1)$ & $10(1)$ \\
\hline $\mathrm{C}(1)$ & $8297(4)$ & $4075(4)$ & $2664(3)$ & $11(1)$ \\
\hline $\mathrm{B}(2)$ & $7957(4)$ & $4067(4)$ & $1505(3)$ & $17(1)$ \\
\hline $\mathrm{B}(3)$ & $7080(4)$ & $5025(4)$ & $2226(3)$ & $15(1)$ \\
\hline $\mathrm{B}(4)$ & $8146(4)$ & $5513(4)$ & $3119(3)$ & $15(1)$ \\
\hline $\mathrm{B}(5)$ & $9696(4)$ & $4860(4)$ & 2954(3) & $16(1)$ \\
\hline $\mathrm{B}(6)$ & $9575(4)$ & $3974(4)$ & 1948(3) & $17(1)$ \\
\hline $\mathrm{B}(7)$ & $9186(4)$ & $4951(5)$ & $1022(3)$ & $22(1)$ \\
\hline $\mathrm{B}(8)$ & $7648(4)$ & $5600(4)$ & 1198(3) & $20(1)$ \\
\hline $\mathrm{B}(9)$ & $7767(4)$ & $6492(4)$ & $2200(3)$ & $20(1)$ \\
\hline $\mathrm{B}(10)$ & $9390(4)$ & $6393(4)$ & $2656(3)$ & $20(1)$ \\
\hline $\mathrm{B}(11)$ & $10267(4)$ & $5438(4)$ & 1922(3) & $20(1)$ \\
\hline $\mathrm{B}(12)$ & $9070(4)$ & $6451(4)$ & $1459(3)$ & $23(1)$ \\
\hline $\mathrm{C}(2)$ & $8776(3)$ & $2755(6)$ & $4420(2)$ & $14(1)$ \\
\hline $\mathrm{C}(3)$ & $8552(5)$ & $1589(5)$ & $4960(3)$ & $19(1)$ \\
\hline$C(4)$ & $8697(4)$ & $3837(5)$ & $5067(3)$ & $17(1)$ \\
\hline$C(5)$ & $8360(5)$ & $1379(5)$ & 2768(4) & $19(1)$ \\
\hline $\mathrm{C}(6)$ & $7580(5)$ & 234(3) & $2962(3)$ & $25(1)$ \\
\hline
\end{tabular}




$\begin{array}{lcccc}\mathrm{C}(7) & 9812(5) & 1106(5) & 2915(4) & 28(1) \\ \mathrm{N}(1 \mathrm{C}) & 6714(3) & 9300(3) & 9912(2) & 17(1) \\ \mathrm{C}(1 \mathrm{C}) & 7471(4) & 8323(4) & 9475(3) & 20(1) \\ \mathrm{C}(2 \mathrm{C}) & 7608(4) & 10189(5) & 10363(4) & 40(1) \\ \mathrm{C}(3 \mathrm{C}) & 5898(4) & 9931(4) & 9206(3) & 28(1) \\ \mathrm{C}(4 \mathrm{C}) & 5855(6) & 8750(5) & 10581(4) & 39(1) \\ \mathrm{O}(1 \mathrm{~S}) & 3448(15) & 7529(16) & 1419(13) & 29(2) \\ \mathrm{C}(1 S) & 3817(11) & 7650(14) & 2349(9) & 50(2) \\ \mathrm{C}(2 S) & 3576(12) & 8948(9) & 2582(6) & 60(3) \\ \mathrm{C}(3 S) & 2624(10) & 9388(10) & 1881(9) & 61(2) \\ \mathrm{C}(4 S) & 2485(14) & 8413(13) & 1204(12) & 61(2) \\ \mathrm{O}(1 \mathrm{D}) & 3680(30) & 7680(30) & 1530(20) & 34(5) \\ \mathrm{C}(1 \mathrm{D}) & 2590(30) & 8210(20) & 1079(14) & 42(3) \\ \mathrm{C}(2 \mathrm{D}) & 2093(19) & 9171(18) & 1646(13) & 61(2) \\ \mathrm{C}(3 \mathrm{D}) & 2611(18) & 8927(15) & 2540(10) & 42(3) \\ \mathrm{C}(4 \mathrm{D}) & 3490(20) & 7880(30) & 2464(18) & 50(2)\end{array}$


Table 9. Bond lengths $[\AA]$ and angles $\left[^{\circ}\right]$ for vL147JE_0m.

\begin{tabular}{|c|c|c|c|}
\hline $\operatorname{Ir}(1)-C(5 A)$ & $2.093(3)$ & $\mathrm{B}(2)-\mathrm{B}(7)$ & $1.778(6)$ \\
\hline $\operatorname{Ir}(1)-C(6 A)$ & $2.130(3)$ & $\mathrm{B}(2)-\mathrm{B}(3)$ & $1.779(6)$ \\
\hline $\operatorname{Ir}(1)-C(1 \mathrm{~A})$ & $2.184(4)$ & $\mathrm{B}(2)-\mathrm{B}(8)$ & $1.779(6)$ \\
\hline $\operatorname{Ir}(1)-C(2 A)$ & $2.199(4)$ & $\mathrm{B}(2)-\mathrm{B}(6)$ & $1.779(6)$ \\
\hline $\operatorname{Ir}(1)-\mathrm{P}(1)$ & $2.3709(7)$ & $\mathrm{B}(2)-\mathrm{H}(2)$ & 1.1200 \\
\hline $\operatorname{Ir}(1)-\mathrm{Cl}(1)$ & $2.3941(8)$ & $\mathrm{B}(3)-\mathrm{B}(8)$ & $1.770(6)$ \\
\hline$C(1 A)-C(2 A)$ & $1.401(5)$ & $\mathrm{B}(3)-\mathrm{B}(4)$ & $1.771(6)$ \\
\hline $\mathrm{C}(1 \mathrm{~A})-\mathrm{C}(8 \mathrm{~A})$ & $1.507(6)$ & $\mathrm{B}(3)-\mathrm{B}(9)$ & $1.771(6)$ \\
\hline $\mathrm{C}(1 \mathrm{~A})-\mathrm{H}(1 \mathrm{~A})$ & $0.93(3)$ & $\mathrm{B}(3)-\mathrm{H}(3)$ & 1.1200 \\
\hline $\mathrm{C}(2 \mathrm{~A})-\mathrm{C}(3 \mathrm{~A})$ & $1.516(6)$ & $\mathrm{B}(4)-\mathrm{B}(9)$ & $1.768(6)$ \\
\hline $\mathrm{C}(2 \mathrm{~A})-\mathrm{H}(2 \mathrm{~A})$ & $0.94(3)$ & $\mathrm{B}(4)-\mathrm{B}(10)$ & $1.775(6)$ \\
\hline$C(3 A)-C(4 A)$ & $1.541(5)$ & $\mathrm{B}(4)-\mathrm{B}(5)$ & $1.788(6)$ \\
\hline $\mathrm{C}(3 \mathrm{~A})-\mathrm{H}(3 \mathrm{~A})$ & 0.9900 & $\mathrm{~B}(4)-\mathrm{H}(4)$ & 1.1200 \\
\hline $\mathrm{C}(3 \mathrm{~A})-\mathrm{H}(3 \mathrm{~B})$ & 0.9900 & $\mathrm{~B}(5)-\mathrm{B}(10)$ & $1.776(6)$ \\
\hline $\mathrm{C}(4 \mathrm{~A})-\mathrm{C}(5 \mathrm{~A})$ & $1.512(5)$ & $\mathrm{B}(5)-\mathrm{B}(11)$ & $1.778(6)$ \\
\hline $\mathrm{C}(4 \mathrm{~A})-\mathrm{H}(4 \mathrm{~A})$ & 0.9900 & $\mathrm{~B}(5)-\mathrm{B}(6)$ & $1.781(7)$ \\
\hline $\mathrm{C}(4 \mathrm{~A})-\mathrm{H}(4 \mathrm{~B})$ & 0.9900 & $\mathrm{~B}(5)-\mathrm{H}(5)$ & 1.1200 \\
\hline$C(5 A)-C(6 A)$ & $1.422(5)$ & $\mathrm{B}(6)-\mathrm{B}(11)$ & $1.771(6)$ \\
\hline $\mathrm{C}(5 \mathrm{~A})-\mathrm{H}(5 \mathrm{~A})$ & $0.93(3)$ & $\mathrm{B}(6)-\mathrm{B}(7)$ & $1.776(6)$ \\
\hline$C(6 A)-C(7 A)$ & $1.532(5)$ & $\mathrm{B}(6)-\mathrm{H}(6)$ & 1.1200 \\
\hline $\mathrm{C}(6 \mathrm{~A})-\mathrm{H}(6 \mathrm{~A})$ & $0.94(3)$ & $\mathrm{B}(7)-\mathrm{B}(8)$ & $1.778(7)$ \\
\hline $\mathrm{C}(7 \mathrm{~A})-\mathrm{C}(8 \mathrm{~A})$ & $1.532(6)$ & $\mathrm{B}(7)-\mathrm{B}(12)$ & $1.785(7)$ \\
\hline $\mathrm{C}(7 \mathrm{~A})-\mathrm{H}(7 \mathrm{~A})$ & 0.9900 & $\mathrm{~B}(7)-\mathrm{B}(11)$ & $1.787(6)$ \\
\hline $\mathrm{C}(7 \mathrm{~A})-\mathrm{H}(7 \mathrm{~B})$ & 0.9900 & $\mathrm{~B}(7)-\mathrm{H}(7)$ & 1.1200 \\
\hline $\mathrm{C}(8 \mathrm{~A})-\mathrm{H}(8 \mathrm{~A})$ & 0.9900 & $\mathrm{~B}(8)-\mathrm{B}(12)$ & $1.778(6)$ \\
\hline $\mathrm{C}(8 \mathrm{~A})-\mathrm{H}(8 \mathrm{~B})$ & 0.9900 & $\mathrm{~B}(8)-\mathrm{B}(9)$ & $1.779(7)$ \\
\hline $\mathrm{P}(1)-\mathrm{C}(5)$ & $1.869(5)$ & $\mathrm{B}(8)-\mathrm{H}(8)$ & 1.1200 \\
\hline $\mathrm{P}(1)-\mathrm{C}(2)$ & $1.874(3)$ & $\mathrm{B}(9)-\mathrm{B}(12)$ & $1.775(7)$ \\
\hline $\mathrm{P}(1)-\mathrm{C}(1)$ & $1.885(5)$ & $\mathrm{B}(9)-\mathrm{B}(10)$ & $1.791(6)$ \\
\hline $\mathrm{C}(1)-\mathrm{B}(5)$ & $1.729(6)$ & $\mathrm{B}(9)-\mathrm{H}(9)$ & 1.1200 \\
\hline$C(1)-B(6)$ & $1.734(6)$ & $\mathrm{B}(10)-\mathrm{B}(12)$ & $1.788(6)$ \\
\hline $\mathrm{C}(1)-\mathrm{B}(2)$ & $1.735(6)$ & $\mathrm{B}(10)-\mathrm{B}(11)$ & $1.789(7)$ \\
\hline $\mathrm{C}(1)-\mathrm{B}(4)$ & $1.735(6)$ & $\mathrm{B}(10)-\mathrm{H}(10)$ & 1.1200 \\
\hline $\mathrm{C}(1)-\mathrm{B}(3)$ & $1.746(6)$ & $\mathrm{B}(11)-\mathrm{B}(12)$ & $1.786(6)$ \\
\hline
\end{tabular}




\begin{tabular}{|c|c|c|c|}
\hline $\mathrm{B}(11)-\mathrm{H}(11)$ & 1.1200 & $\mathrm{O}(1 \mathrm{~S})-\mathrm{C}(1 \mathrm{~S})$ & $1.418(19)$ \\
\hline $\mathrm{B}(12)-\mathrm{H}(12)$ & 1.1200 & $\mathrm{O}(1 \mathrm{~S})-\mathrm{C}(4 \mathrm{~S})$ & $1.423(18)$ \\
\hline$C(2)-C(4)$ & $1.534(7)$ & $C(1 S)-C(2 S)$ & $1.498(15)$ \\
\hline$C(2)-C(3)$ & $1.539(7)$ & $\mathrm{C}(1 \mathrm{~S})-\mathrm{H}(1 \mathrm{E})$ & 0.9900 \\
\hline $\mathrm{C}(2)-\mathrm{H}(2 \mathrm{~B})$ & 1.0000 & $\mathrm{C}(1 \mathrm{~S})-\mathrm{H}(1 \mathrm{~F})$ & 0.9900 \\
\hline $\mathrm{C}(3)-\mathrm{H}(3 \mathrm{C})$ & 0.9800 & $C(2 S)-C(3 S)$ & $1.483(13)$ \\
\hline $\mathrm{C}(3)-\mathrm{H}(3 \mathrm{D})$ & 0.9800 & $\mathrm{C}(2 \mathrm{~S})-\mathrm{H}(2 \mathrm{~F})$ & 0.9900 \\
\hline $\mathrm{C}(3)-\mathrm{H}(3 \mathrm{E})$ & 0.9800 & $\mathrm{C}(2 \mathrm{~S})-\mathrm{H}(2 \mathrm{G})$ & 0.9900 \\
\hline $\mathrm{C}(4)-\mathrm{H}(4 \mathrm{C})$ & 0.9800 & $C(3 S)-C(4 S)$ & $1.474(13)$ \\
\hline $\mathrm{C}(4)-\mathrm{H}(4 \mathrm{D})$ & 0.9800 & $\mathrm{C}(3 \mathrm{~S})-\mathrm{H}(3 \mathrm{I})$ & 0.9900 \\
\hline $\mathrm{C}(4)-\mathrm{H}(4 \mathrm{E})$ & 0.9800 & $\mathrm{C}(3 \mathrm{~S})-\mathrm{H}(3 \mathrm{~J})$ & 0.9900 \\
\hline$C(5)-C(6)$ & $1.535(7)$ & $\mathrm{C}(4 \mathrm{~S})-\mathrm{H}(4 \mathrm{I})$ & 0.9900 \\
\hline$C(5)-C(7)$ & $1.543(7)$ & $\mathrm{C}(4 \mathrm{~S})-\mathrm{H}(4 \mathrm{~J})$ & 0.9900 \\
\hline $\mathrm{C}(5)-\mathrm{H}(5 \mathrm{~B})$ & 1.0000 & $\mathrm{O}(1 \mathrm{D})-\mathrm{C}(1 \mathrm{D})$ & $1.42(3)$ \\
\hline $\mathrm{C}(6)-\mathrm{H}(6 \mathrm{~B})$ & 0.9800 & $\mathrm{O}(1 \mathrm{D})-\mathrm{C}(4 \mathrm{D})$ & $1.43(3)$ \\
\hline $\mathrm{C}(6)-\mathrm{H}(6 \mathrm{C})$ & 0.9800 & $\mathrm{C}(1 \mathrm{D})-\mathrm{C}(2 \mathrm{D})$ & $1.456(16)$ \\
\hline $\mathrm{C}(6)-\mathrm{H}(6 \mathrm{D})$ & 0.9800 & $\mathrm{C}(1 \mathrm{D})-\mathrm{H}(1 \mathrm{G})$ & 0.9900 \\
\hline $\mathrm{C}(7)-\mathrm{H}(7 \mathrm{C})$ & 0.9800 & $\mathrm{C}(1 \mathrm{D})-\mathrm{H}(1 \mathrm{H})$ & 0.9900 \\
\hline $\mathrm{C}(7)-\mathrm{H}(7 \mathrm{D})$ & 0.9800 & $\mathrm{C}(2 \mathrm{D})-\mathrm{C}(3 \mathrm{D})$ & $1.432(17)$ \\
\hline $\mathrm{C}(7)-\mathrm{H}(7 \mathrm{E})$ & 0.9800 & $\mathrm{C}(2 \mathrm{D})-\mathrm{H}(2 \mathrm{H})$ & 0.9900 \\
\hline $\mathrm{N}(1 \mathrm{C})-\mathrm{C}(4 \mathrm{C})$ & $1.488(6)$ & $\mathrm{C}(2 \mathrm{D})-\mathrm{H}(2 \mathrm{I})$ & 0.9900 \\
\hline $\mathrm{N}(1 \mathrm{C})-\mathrm{C}(2 \mathrm{C})$ & $1.489(5)$ & $C(3 D)-C(4 D)$ & $1.477(18)$ \\
\hline $\mathrm{N}(1 \mathrm{C})-\mathrm{C}(3 \mathrm{C})$ & $1.490(5)$ & $\mathrm{C}(3 \mathrm{D})-\mathrm{H}(3 \mathrm{~K})$ & 0.9900 \\
\hline $\mathrm{N}(1 \mathrm{C})-\mathrm{C}(1 \mathrm{C})$ & $1.496(5)$ & $\mathrm{C}(3 \mathrm{D})-\mathrm{H}(3 \mathrm{~L})$ & 0.9900 \\
\hline $\mathrm{C}(1 \mathrm{C})-\mathrm{H}(1 \mathrm{~B})$ & 0.9800 & $\mathrm{C}(4 \mathrm{D})-\mathrm{H}(4 \mathrm{~K})$ & 0.9900 \\
\hline $\mathrm{C}(1 \mathrm{C})-\mathrm{H}(1 \mathrm{C})$ & 0.9800 & $\mathrm{C}(4 \mathrm{D})-\mathrm{H}(4 \mathrm{~L})$ & 0.9900 \\
\hline $\mathrm{C}(1 \mathrm{C})-\mathrm{H}(1 \mathrm{D})$ & 0.9800 & & \\
\hline $\mathrm{C}(2 \mathrm{C})-\mathrm{H}(2 \mathrm{C})$ & 0.9800 & $C(5 A)-\operatorname{Ir}(1)-C(6 A)$ & $39.32(13)$ \\
\hline $\mathrm{C}(2 \mathrm{C})-\mathrm{H}(2 \mathrm{D})$ & 0.9800 & $C(5 A)-\operatorname{Ir}(1)-C(1 A)$ & $97.17(15)$ \\
\hline $\mathrm{C}(2 \mathrm{C})-\mathrm{H}(2 \mathrm{E})$ & 0.9800 & $C(6 A)-\operatorname{Ir}(1)-C(1 A)$ & $80.33(15)$ \\
\hline $\mathrm{C}(3 \mathrm{C})-\mathrm{H}(3 \mathrm{~F})$ & 0.9800 & $\mathrm{C}(5 \mathrm{~A})-\operatorname{Ir}(1)-\mathrm{C}(2 \mathrm{~A})$ & $80.93(14)$ \\
\hline $\mathrm{C}(3 \mathrm{C})-\mathrm{H}(3 \mathrm{G})$ & 0.9800 & $\mathrm{C}(6 \mathrm{~A})-\operatorname{Ir}(1)-\mathrm{C}(2 \mathrm{~A})$ & $88.50(14)$ \\
\hline $\mathrm{C}(3 \mathrm{C})-\mathrm{H}(3 \mathrm{H})$ & 0.9800 & $\mathrm{C}(1 \mathrm{~A})-\operatorname{Ir}(1)-\mathrm{C}(2 \mathrm{~A})$ & $37.28(13)$ \\
\hline $\mathrm{C}(4 \mathrm{C})-\mathrm{H}(4 \mathrm{~F})$ & 0.9800 & $\mathrm{C}(5 \mathrm{~A})-\operatorname{Ir}(1)-\mathrm{P}(1)$ & $92.42(10)$ \\
\hline $\mathrm{C}(4 \mathrm{C})-\mathrm{H}(4 \mathrm{G})$ & 0.9800 & $\mathrm{C}(6 \mathrm{~A})-\operatorname{Ir}(1)-\mathrm{P}(1)$ & $97.24(9)$ \\
\hline $\mathrm{C}(4 \mathrm{C})-\mathrm{H}(4 \mathrm{H})$ & 0.9800 & $\mathrm{C}(1 \mathrm{~A})-\operatorname{Ir}(1)-\mathrm{P}(1)$ & $161.05(12)$ \\
\hline
\end{tabular}




\begin{tabular}{|c|c|c|c|}
\hline $\mathrm{C}(2 \mathrm{~A})-\operatorname{Ir}(1)-\mathrm{P}(1)$ & $161.56(11)$ & $C(5 A)-C(6 A)-C(7 A)$ & $122.5(3)$ \\
\hline $\mathrm{C}(5 \mathrm{~A})-\operatorname{Ir}(1)-\mathrm{Cl}(1)$ & $155.66(10)$ & $C(5 A)-C(6 A)-\operatorname{Ir}(1)$ & $68.93(19)$ \\
\hline $\mathrm{C}(6 \mathrm{~A})-\mathrm{Ir}(1)-\mathrm{Cl}(1)$ & $162.58(9)$ & $C(7 A)-C(6 A)-\operatorname{Ir}(1)$ & $114.9(2)$ \\
\hline $\mathrm{C}(1 \mathrm{~A})-\operatorname{Ir}(1)-\mathrm{Cl}(1)$ & $86.56(11)$ & $\mathrm{C}(5 \mathrm{~A})-\mathrm{C}(6 \mathrm{~A})-\mathrm{H}(6 \mathrm{~A})$ & $115(4)$ \\
\hline $\mathrm{C}(2 \mathrm{~A})-\mathrm{Ir}(1)-\mathrm{Cl}(1)$ & $87.95(11)$ & $C(7 A)-C(6 A)-H(6 A)$ & 113(3) \\
\hline $\mathrm{P}(1)-\operatorname{Ir}(1)-\mathrm{Cl}(1)$ & $91.43(3)$ & $\operatorname{Ir}(1)-C(6 A)-H(6 A)$ & $115(3)$ \\
\hline $\mathrm{C}(2 \mathrm{~A})-\mathrm{C}(1 \mathrm{~A})-\mathrm{C}(8 \mathrm{~A})$ & $124.4(4)$ & $C(6 A)-C(7 A)-C(8 A)$ & 111.3(3) \\
\hline $\mathrm{C}(2 \mathrm{~A})-\mathrm{C}(1 \mathrm{~A})-\operatorname{Ir}(1)$ & $71.9(3)$ & $\mathrm{C}(6 \mathrm{~A})-\mathrm{C}(7 \mathrm{~A})-\mathrm{H}(7 \mathrm{~A})$ & 109.4 \\
\hline $\mathrm{C}(8 \mathrm{~A})-\mathrm{C}(1 \mathrm{~A})-\operatorname{Ir}(1)$ & $110.6(3)$ & $\mathrm{C}(8 \mathrm{~A})-\mathrm{C}(7 \mathrm{~A})-\mathrm{H}(7 \mathrm{~A})$ & 109.4 \\
\hline $\mathrm{C}(2 \mathrm{~A})-\mathrm{C}(1 \mathrm{~A})-\mathrm{H}(1 \mathrm{~A})$ & $120(3)$ & $\mathrm{C}(6 \mathrm{~A})-\mathrm{C}(7 \mathrm{~A})-\mathrm{H}(7 \mathrm{~B})$ & 109.4 \\
\hline $\mathrm{C}(8 \mathrm{~A})-\mathrm{C}(1 \mathrm{~A})-\mathrm{H}(1 \mathrm{~A})$ & 112(3) & $\mathrm{C}(8 \mathrm{~A})-\mathrm{C}(7 \mathrm{~A})-\mathrm{H}(7 \mathrm{~B})$ & 109.4 \\
\hline $\operatorname{Ir}(1)-C(1 \mathrm{~A})-\mathrm{H}(1 \mathrm{~A})$ & $108(3)$ & $\mathrm{H}(7 \mathrm{~A})-\mathrm{C}(7 \mathrm{~A})-\mathrm{H}(7 \mathrm{~B})$ & 108.0 \\
\hline $\mathrm{C}(1 \mathrm{~A})-\mathrm{C}(2 \mathrm{~A})-\mathrm{C}(3 \mathrm{~A})$ & $124.0(3)$ & $\mathrm{C}(1 \mathrm{~A})-\mathrm{C}(8 \mathrm{~A})-\mathrm{C}(7 \mathrm{~A})$ & $112.7(3)$ \\
\hline $\mathrm{C}(1 \mathrm{~A})-\mathrm{C}(2 \mathrm{~A})-\operatorname{Ir}(1)$ & $70.8(3)$ & $\mathrm{C}(1 \mathrm{~A})-\mathrm{C}(8 \mathrm{~A})-\mathrm{H}(8 \mathrm{~A})$ & 109.1 \\
\hline $\mathrm{C}(3 \mathrm{~A})-\mathrm{C}(2 \mathrm{~A})-\operatorname{Ir}(1)$ & $112.6(3)$ & $\mathrm{C}(7 \mathrm{~A})-\mathrm{C}(8 \mathrm{~A})-\mathrm{H}(8 \mathrm{~A})$ & 109.1 \\
\hline$C(1 A)-C(2 A)-H(2 A)$ & 119(3) & $\mathrm{C}(1 \mathrm{~A})-\mathrm{C}(8 \mathrm{~A})-\mathrm{H}(8 \mathrm{~B})$ & 109.1 \\
\hline $\mathrm{C}(3 \mathrm{~A})-\mathrm{C}(2 \mathrm{~A})-\mathrm{H}(2 \mathrm{~A})$ & $113(3)$ & $\mathrm{C}(7 \mathrm{~A})-\mathrm{C}(8 \mathrm{~A})-\mathrm{H}(8 \mathrm{~B})$ & 109.1 \\
\hline $\operatorname{Ir}(1)-C(2 A)-H(2 A)$ & $105(3)$ & $\mathrm{H}(8 \mathrm{~A})-\mathrm{C}(8 \mathrm{~A})-\mathrm{H}(8 \mathrm{~B})$ & 107.8 \\
\hline$C(2 A)-C(3 A)-C(4 A)$ & $111.7(3)$ & $\mathrm{C}(5)-\mathrm{P}(1)-\mathrm{C}(2)$ & $101.3(2)$ \\
\hline $\mathrm{C}(2 \mathrm{~A})-\mathrm{C}(3 \mathrm{~A})-\mathrm{H}(3 \mathrm{~A})$ & 109.3 & $\mathrm{C}(5)-\mathrm{P}(1)-\mathrm{C}(1)$ & $105.20(16)$ \\
\hline $\mathrm{C}(4 \mathrm{~A})-\mathrm{C}(3 \mathrm{~A})-\mathrm{H}(3 \mathrm{~A})$ & 109.3 & $\mathrm{C}(2)-\mathrm{P}(1)-\mathrm{C}(1)$ & $105.9(2)$ \\
\hline $\mathrm{C}(2 \mathrm{~A})-\mathrm{C}(3 \mathrm{~A})-\mathrm{H}(3 \mathrm{~B})$ & 109.3 & $\mathrm{C}(5)-\mathrm{P}(1)-\operatorname{Ir}(1)$ & $111.75(18)$ \\
\hline $\mathrm{C}(4 \mathrm{~A})-\mathrm{C}(3 \mathrm{~A})-\mathrm{H}(3 \mathrm{~B})$ & 109.3 & $\mathrm{C}(2)-\mathrm{P}(1)-\operatorname{Ir}(1)$ & $123.07(9)$ \\
\hline $\mathrm{H}(3 \mathrm{~A})-\mathrm{C}(3 \mathrm{~A})-\mathrm{H}(3 \mathrm{~B})$ & 107.9 & $\mathrm{C}(1)-\mathrm{P}(1)-\operatorname{Ir}(1)$ & $108.15(14)$ \\
\hline $\mathrm{C}(5 \mathrm{~A})-\mathrm{C}(4 \mathrm{~A})-\mathrm{C}(3 \mathrm{~A})$ & $112.8(3)$ & $\mathrm{B}(5)-\mathrm{C}(1)-\mathrm{B}(6)$ & $61.9(3)$ \\
\hline$C(5 A)-C(4 A)-H(4 A)$ & 109.0 & $\mathrm{~B}(5)-\mathrm{C}(1)-\mathrm{B}(2)$ & $112.5(3)$ \\
\hline $\mathrm{C}(3 \mathrm{~A})-\mathrm{C}(4 \mathrm{~A})-\mathrm{H}(4 \mathrm{~A})$ & 109.0 & $\mathrm{~B}(6)-\mathrm{C}(1)-\mathrm{B}(2)$ & $61.7(3)$ \\
\hline $\mathrm{C}(5 \mathrm{~A})-\mathrm{C}(4 \mathrm{~A})-\mathrm{H}(4 \mathrm{~B})$ & 109.0 & $\mathrm{~B}(5)-\mathrm{C}(1)-\mathrm{B}(4)$ & $62.1(3)$ \\
\hline $\mathrm{C}(3 \mathrm{~A})-\mathrm{C}(4 \mathrm{~A})-\mathrm{H}(4 \mathrm{~B})$ & 109.0 & $\mathrm{~B}(6)-\mathrm{C}(1)-\mathrm{B}(4)$ & $112.3(3)$ \\
\hline $\mathrm{H}(4 \mathrm{~A})-\mathrm{C}(4 \mathrm{~A})-\mathrm{H}(4 \mathrm{~B})$ & 107.8 & $\mathrm{~B}(2)-\mathrm{C}(1)-\mathrm{B}(4)$ & $111.7(3)$ \\
\hline$C(6 A)-C(5 A)-C(4 A)$ & $124.3(3)$ & $\mathrm{B}(5)-\mathrm{C}(1)-\mathrm{B}(3)$ & 112.2(3) \\
\hline$C(6 A)-C(5 A)-\operatorname{Ir}(1)$ & $71.7(2)$ & $\mathrm{B}(6)-\mathrm{C}(1)-\mathrm{B}(3)$ & $111.8(3)$ \\
\hline $\mathrm{C}(4 \mathrm{~A})-\mathrm{C}(5 \mathrm{~A})-\operatorname{Ir}(1)$ & $112.3(2)$ & $\mathrm{B}(2)-\mathrm{C}(1)-\mathrm{B}(3)$ & $61.5(2)$ \\
\hline $\mathrm{C}(6 \mathrm{~A})-\mathrm{C}(5 \mathrm{~A})-\mathrm{H}(5 \mathrm{~A})$ & $118(3)$ & $\mathrm{B}(4)-\mathrm{C}(1)-\mathrm{B}(3)$ & $61.2(2)$ \\
\hline $\mathrm{C}(4 \mathrm{~A})-\mathrm{C}(5 \mathrm{~A})-\mathrm{H}(5 \mathrm{~A})$ & 112(3) & $\mathrm{B}(5)-\mathrm{C}(1)-\mathrm{P}(1)$ & $121.9(3)$ \\
\hline $\operatorname{Ir}(1)-C(5 A)-H(5 A)$ & $110(3)$ & $\mathrm{B}(6)-\mathrm{C}(1)-\mathrm{P}(1)$ & $122.5(3)$ \\
\hline
\end{tabular}




\begin{tabular}{|c|c|c|c|}
\hline $\mathrm{B}(2)-\mathrm{C}(1)-\mathrm{P}(1)$ & $118.3(3)$ & $\mathrm{C}(1)-\mathrm{B}(4)-\mathrm{B}(10)$ & $105.9(3)$ \\
\hline $\mathrm{B}(4)-\mathrm{C}(1)-\mathrm{P}(1)$ & $117.9(3)$ & $\mathrm{B}(9)-\mathrm{B}(4)-\mathrm{B}(10)$ & $60.7(2)$ \\
\hline $\mathrm{B}(3)-\mathrm{C}(1)-\mathrm{P}(1)$ & $115.7(3)$ & $\mathrm{B}(3)-\mathrm{B}(4)-\mathrm{B}(10)$ & $108.7(3)$ \\
\hline $\mathrm{C}(1)-\mathrm{B}(2)-\mathrm{B}(7)$ & $105.9(3)$ & $\mathrm{C}(1)-\mathrm{B}(4)-\mathrm{B}(5)$ & $58.7(2)$ \\
\hline $\mathrm{C}(1)-\mathrm{B}(2)-\mathrm{B}(3)$ & $59.6(2)$ & $\mathrm{B}(9)-\mathrm{B}(4)-\mathrm{B}(5)$ & $108.4(3)$ \\
\hline $\mathrm{B}(7)-\mathrm{B}(2)-\mathrm{B}(3)$ & $107.9(3)$ & $\mathrm{B}(3)-\mathrm{B}(4)-\mathrm{B}(5)$ & $108.2(3)$ \\
\hline $\mathrm{C}(1)-\mathrm{B}(2)-\mathrm{B}(8)$ & $105.9(3)$ & $\mathrm{B}(10)-\mathrm{B}(4)-\mathrm{B}(5)$ & $59.8(3)$ \\
\hline $\mathrm{B}(7)-\mathrm{B}(2)-\mathrm{B}(8)$ & $60.0(2)$ & $\mathrm{C}(1)-\mathrm{B}(4)-\mathrm{H}(4)$ & 123.6 \\
\hline $\mathrm{B}(3)-\mathrm{B}(2)-\mathrm{B}(8)$ & $59.7(2)$ & $\mathrm{B}(9)-\mathrm{B}(4)-\mathrm{H}(4)$ & 121.6 \\
\hline $\mathrm{C}(1)-\mathrm{B}(2)-\mathrm{B}(6)$ & $59.1(2)$ & $\mathrm{B}(3)-\mathrm{B}(4)-\mathrm{H}(4)$ & 121.1 \\
\hline $\mathrm{B}(7)-\mathrm{B}(2)-\mathrm{B}(6)$ & $59.9(2)$ & $\mathrm{B}(10)-\mathrm{B}(4)-\mathrm{H}(4)$ & 121.8 \\
\hline $\mathrm{B}(3)-\mathrm{B}(2)-\mathrm{B}(6)$ & $108.2(3)$ & $\mathrm{B}(5)-\mathrm{B}(4)-\mathrm{H}(4)$ & 121.8 \\
\hline $\mathrm{B}(8)-\mathrm{B}(2)-\mathrm{B}(6)$ & $107.8(3)$ & $\mathrm{C}(1)-\mathrm{B}(5)-\mathrm{B}(10)$ & $106.1(3)$ \\
\hline $\mathrm{C}(1)-\mathrm{B}(2)-\mathrm{H}(2)$ & 123.5 & $\mathrm{C}(1)-\mathrm{B}(5)-\mathrm{B}(11)$ & $105.8(3)$ \\
\hline $\mathrm{B}(7)-\mathrm{B}(2)-\mathrm{H}(2)$ & 122.2 & $\mathrm{~B}(10)-\mathrm{B}(5)-\mathrm{B}(11)$ & $60.4(3)$ \\
\hline $\mathrm{B}(3)-\mathrm{B}(2)-\mathrm{H}(2)$ & 121.5 & $\mathrm{C}(1)-\mathrm{B}(5)-\mathrm{B}(6)$ & $59.2(2)$ \\
\hline $\mathrm{B}(8)-\mathrm{B}(2)-\mathrm{H}(2)$ & 122.3 & $\mathrm{~B}(10)-\mathrm{B}(5)-\mathrm{B}(6)$ & $108.2(3)$ \\
\hline $\mathrm{B}(6)-\mathrm{B}(2)-\mathrm{H}(2)$ & 121.6 & $\mathrm{~B}(11)-\mathrm{B}(5)-\mathrm{B}(6)$ & $59.7(3)$ \\
\hline $\mathrm{C}(1)-\mathrm{B}(3)-\mathrm{B}(8)$ & $105.8(3)$ & $\mathrm{C}(1)-\mathrm{B}(5)-\mathrm{B}(4)$ & $59.1(3)$ \\
\hline $\mathrm{C}(1)-\mathrm{B}(3)-\mathrm{B}(4)$ & $59.1(2)$ & $\mathrm{B}(10)-\mathrm{B}(5)-\mathrm{B}(4)$ & $59.8(2)$ \\
\hline $\mathrm{B}(8)-\mathrm{B}(3)-\mathrm{B}(4)$ & $108.1(3)$ & $\mathrm{B}(11)-\mathrm{B}(5)-\mathrm{B}(4)$ & $107.8(3)$ \\
\hline $\mathrm{C}(1)-\mathrm{B}(3)-\mathrm{B}(9)$ & $105.8(3)$ & $\mathrm{B}(6)-\mathrm{B}(5)-\mathrm{B}(4)$ & 107.7(3) \\
\hline $\mathrm{B}(8)-\mathrm{B}(3)-\mathrm{B}(9)$ & $60.3(2)$ & $\mathrm{C}(1)-\mathrm{B}(5)-\mathrm{H}(5)$ & 123.6 \\
\hline $\mathrm{B}(4)-\mathrm{B}(3)-\mathrm{B}(9)$ & $59.9(2)$ & $\mathrm{B}(10)-\mathrm{B}(5)-\mathrm{H}(5)$ & 121.8 \\
\hline $\mathrm{C}(1)-\mathrm{B}(3)-\mathrm{B}(2)$ & $59.0(2)$ & $\mathrm{B}(11)-\mathrm{B}(5)-\mathrm{H}(5)$ & 122.2 \\
\hline $\mathrm{B}(8)-\mathrm{B}(3)-\mathrm{B}(2)$ & $60.2(2)$ & $\mathrm{B}(6)-\mathrm{B}(5)-\mathrm{H}(5)$ & 121.7 \\
\hline $\mathrm{B}(4)-\mathrm{B}(3)-\mathrm{B}(2)$ & $108.0(3)$ & $\mathrm{B}(4)-\mathrm{B}(5)-\mathrm{H}(5)$ & 121.9 \\
\hline $\mathrm{B}(9)-\mathrm{B}(3)-\mathrm{B}(2)$ & $108.4(3)$ & $\mathrm{C}(1)-\mathrm{B}(6)-\mathrm{B}(11)$ & $105.9(3)$ \\
\hline $\mathrm{C}(1)-\mathrm{B}(3)-\mathrm{H}(3)$ & 123.9 & $\mathrm{C}(1)-\mathrm{B}(6)-\mathrm{B}(7)$ & $106.0(3)$ \\
\hline $\mathrm{B}(8)-\mathrm{B}(3)-\mathrm{H}(3)$ & 122.0 & $\mathrm{~B}(11)-\mathrm{B}(6)-\mathrm{B}(7)$ & $60.5(2)$ \\
\hline $\mathrm{B}(4)-\mathrm{B}(3)-\mathrm{H}(3)$ & 121.6 & $\mathrm{C}(1)-\mathrm{B}(6)-\mathrm{B}(2)$ & $59.2(2)$ \\
\hline $\mathrm{B}(9)-\mathrm{B}(3)-\mathrm{H}(3)$ & 122.0 & $\mathrm{~B}(11)-\mathrm{B}(6)-\mathrm{B}(2)$ & $108.4(3)$ \\
\hline $\mathrm{B}(2)-\mathrm{B}(3)-\mathrm{H}(3)$ & 121.4 & $\mathrm{~B}(7)-\mathrm{B}(6)-\mathrm{B}(2)$ & $60.0(2)$ \\
\hline $\mathrm{C}(1)-\mathrm{B}(4)-\mathrm{B}(9)$ & $106.4(3)$ & $\mathrm{C}(1)-\mathrm{B}(6)-\mathrm{B}(5)$ & $58.9(3)$ \\
\hline $\mathrm{C}(1)-\mathrm{B}(4)-\mathrm{B}(3)$ & $59.7(2)$ & $\mathrm{B}(11)-\mathrm{B}(6)-\mathrm{B}(5)$ & $60.1(3)$ \\
\hline $\mathrm{B}(9)-\mathrm{B}(4)-\mathrm{B}(3)$ & $60.1(2)$ & $\mathrm{B}(7)-\mathrm{B}(6)-\mathrm{B}(5)$ & $108.4(3)$ \\
\hline
\end{tabular}




\begin{tabular}{|c|c|c|c|}
\hline $\mathrm{B}(2)-\mathrm{B}(6)-\mathrm{B}(5)$ & $108.0(3)$ & $\mathrm{B}(4)-\mathrm{B}(9)-\mathrm{B}(3)$ & $60.1(2)$ \\
\hline $\mathrm{C}(1)-\mathrm{B}(6)-\mathrm{H}(6)$ & 123.8 & $\mathrm{~B}(4)-\mathrm{B}(9)-\mathrm{B}(12)$ & $107.9(3)$ \\
\hline $\mathrm{B}(11)-\mathrm{B}(6)-\mathrm{H}(6)$ & 121.8 & $\mathrm{~B}(3)-\mathrm{B}(9)-\mathrm{B}(12)$ & $107.9(3)$ \\
\hline $\mathrm{B}(7)-\mathrm{B}(6)-\mathrm{H}(6)$ & 121.8 & $\mathrm{~B}(4)-\mathrm{B}(9)-\mathrm{B}(8)$ & $107.8(3)$ \\
\hline $\mathrm{B}(2)-\mathrm{B}(6)-\mathrm{H}(6)$ & 121.5 & $\mathrm{~B}(3)-\mathrm{B}(9)-\mathrm{B}(8)$ & $59.8(2)$ \\
\hline $\mathrm{B}(5)-\mathrm{B}(6)-\mathrm{H}(6)$ & 121.6 & $\mathrm{~B}(12)-\mathrm{B}(9)-\mathrm{B}(8)$ & $60.0(3)$ \\
\hline $\mathrm{B}(6)-\mathrm{B}(7)-\mathrm{B}(8)$ & $108.0(3)$ & $\mathrm{B}(4)-\mathrm{B}(9)-\mathrm{B}(10)$ & $59.8(2)$ \\
\hline $\mathrm{B}(6)-\mathrm{B}(7)-\mathrm{B}(2)$ & $60.1(2)$ & $\mathrm{B}(3)-\mathrm{B}(9)-\mathrm{B}(10)$ & $108.0(3)$ \\
\hline $\mathrm{B}(8)-\mathrm{B}(7)-\mathrm{B}(2)$ & $60.0(2)$ & $\mathrm{B}(12)-\mathrm{B}(9)-\mathrm{B}(10)$ & $60.2(2)$ \\
\hline $\mathrm{B}(6)-\mathrm{B}(7)-\mathrm{B}(12)$ & $107.6(3)$ & $\mathrm{B}(8)-\mathrm{B}(9)-\mathrm{B}(10)$ & $108.1(3)$ \\
\hline $\mathrm{B}(8)-\mathrm{B}(7)-\mathrm{B}(12)$ & $59.9(3)$ & $\mathrm{B}(4)-\mathrm{B}(9)-\mathrm{H}(9)$ & 121.8 \\
\hline $\mathrm{B}(2)-\mathrm{B}(7)-\mathrm{B}(12)$ & 107.7(3) & $\mathrm{B}(3)-\mathrm{B}(9)-\mathrm{H}(9)$ & 121.8 \\
\hline $\mathrm{B}(6)-\mathrm{B}(7)-\mathrm{B}(11)$ & $59.6(2)$ & $\mathrm{B}(12)-\mathrm{B}(9)-\mathrm{H}(9)$ & 121.7 \\
\hline $\mathrm{B}(8)-\mathrm{B}(7)-\mathrm{B}(11)$ & $107.9(3)$ & $\mathrm{B}(8)-\mathrm{B}(9)-\mathrm{H}(9)$ & 121.8 \\
\hline $\mathrm{B}(2)-\mathrm{B}(7)-\mathrm{B}(11)$ & $107.7(3)$ & $\mathrm{B}(10)-\mathrm{B}(9)-\mathrm{H}(9)$ & 121.6 \\
\hline $\mathrm{B}(12)-\mathrm{B}(7)-\mathrm{B}(11)$ & $60.0(3)$ & $\mathrm{B}(4)-\mathrm{B}(10)-\mathrm{B}(5)$ & $60.5(2)$ \\
\hline $\mathrm{B}(6)-\mathrm{B}(7)-\mathrm{H}(7)$ & 121.9 & $\mathrm{~B}(4)-\mathrm{B}(10)-\mathrm{B}(12)$ & $107.0(3)$ \\
\hline $\mathrm{B}(8)-\mathrm{B}(7)-\mathrm{H}(7)$ & 121.7 & $\mathrm{~B}(5)-\mathrm{B}(10)-\mathrm{B}(12)$ & $107.7(3)$ \\
\hline $\mathrm{B}(2)-\mathrm{B}(7)-\mathrm{H}(7)$ & 121.8 & $\mathrm{~B}(4)-\mathrm{B}(10)-\mathrm{B}(11)$ & $107.8(3)$ \\
\hline $\mathrm{B}(12)-\mathrm{B}(7)-\mathrm{H}(7)$ & 121.9 & $\mathrm{~B}(5)-\mathrm{B}(10)-\mathrm{B}(11)$ & $59.8(3)$ \\
\hline $\mathrm{B}(11)-\mathrm{B}(7)-\mathrm{H}(7)$ & 121.9 & $\mathrm{~B}(12)-\mathrm{B}(10)-\mathrm{B}(11)$ & $59.9(3)$ \\
\hline $\mathrm{B}(3)-\mathrm{B}(8)-\mathrm{B}(7)$ & $108.3(3)$ & $\mathrm{B}(4)-\mathrm{B}(10)-\mathrm{B}(9)$ & $59.4(2)$ \\
\hline $\mathrm{B}(3)-\mathrm{B}(8)-\mathrm{B}(12)$ & $107.8(3)$ & $\mathrm{B}(5)-\mathrm{B}(10)-\mathrm{B}(9)$ & $107.9(3)$ \\
\hline $\mathrm{B}(7)-\mathrm{B}(8)-\mathrm{B}(12)$ & $60.3(3)$ & $\mathrm{B}(12)-\mathrm{B}(10)-\mathrm{B}(9)$ & $59.5(3)$ \\
\hline $\mathrm{B}(3)-\mathrm{B}(8)-\mathrm{B}(2)$ & $60.2(2)$ & $\mathrm{B}(11)-\mathrm{B}(10)-\mathrm{B}(9)$ & $107.5(3)$ \\
\hline $\mathrm{B}(7)-\mathrm{B}(8)-\mathrm{B}(2)$ & $60.0(2)$ & $\mathrm{B}(4)-\mathrm{B}(10)-\mathrm{H}(10)$ & 122.0 \\
\hline $\mathrm{B}(12)-\mathrm{B}(8)-\mathrm{B}(2)$ & $108.0(3)$ & $\mathrm{B}(5)-\mathrm{B}(10)-\mathrm{H}(10)$ & 121.6 \\
\hline $\mathrm{B}(3)-\mathrm{B}(8)-\mathrm{B}(9)$ & $59.9(2)$ & $\mathrm{B}(12)-\mathrm{B}(10)-\mathrm{H}(10)$ & 122.3 \\
\hline $\mathrm{B}(7)-\mathrm{B}(8)-\mathrm{B}(9)$ & $108.3(3)$ & $\mathrm{B}(11)-\mathrm{B}(10)-\mathrm{H}(10)$ & 121.8 \\
\hline $\mathrm{B}(12)-\mathrm{B}(8)-\mathrm{B}(9)$ & $59.9(3)$ & $\mathrm{B}(9)-\mathrm{B}(10)-\mathrm{H}(10)$ & 122.2 \\
\hline $\mathrm{B}(2)-\mathrm{B}(8)-\mathrm{B}(9)$ & $108.0(3)$ & $\mathrm{B}(6)-\mathrm{B}(11)-\mathrm{B}(5)$ & $60.2(2)$ \\
\hline $\mathrm{B}(3)-\mathrm{B}(8)-\mathrm{H}(8)$ & 121.7 & $\mathrm{~B}(6)-\mathrm{B}(11)-\mathrm{B}(12)$ & $107.8(3)$ \\
\hline $\mathrm{B}(7)-\mathrm{B}(8)-\mathrm{H}(8)$ & 121.4 & $\mathrm{~B}(5)-\mathrm{B}(11)-\mathrm{B}(12)$ & $107.7(3)$ \\
\hline $\mathrm{B}(12)-\mathrm{B}(8)-\mathrm{H}(8)$ & 121.8 & $\mathrm{~B}(6)-\mathrm{B}(11)-\mathrm{B}(7)$ & $59.9(2)$ \\
\hline $\mathrm{B}(2)-\mathrm{B}(8)-\mathrm{H}(8)$ & 121.7 & $\mathrm{~B}(5)-\mathrm{B}(11)-\mathrm{B}(7)$ & $108.0(3)$ \\
\hline $\mathrm{B}(9)-\mathrm{B}(8)-\mathrm{H}(8)$ & 121.7 & $\mathrm{~B}(12)-\mathrm{B}(11)-\mathrm{B}(7)$ & $59.9(3)$ \\
\hline
\end{tabular}




\begin{tabular}{|c|c|c|c|}
\hline $\mathrm{B}(6)-\mathrm{B}(11)-\mathrm{B}(10)$ & $108.0(3)$ & $\mathrm{C}(2)-\mathrm{C}(4)-\mathrm{H}(4 \mathrm{C})$ & 109.5 \\
\hline $\mathrm{B}(5)-\mathrm{B}(11)-\mathrm{B}(10)$ & $59.7(3)$ & $\mathrm{C}(2)-\mathrm{C}(4)-\mathrm{H}(4 \mathrm{D})$ & 109.5 \\
\hline $\mathrm{B}(12)-\mathrm{B}(11)-\mathrm{B}(10)$ & $60.0(3)$ & $\mathrm{H}(4 \mathrm{C})-\mathrm{C}(4)-\mathrm{H}(4 \mathrm{D})$ & 109.5 \\
\hline $\mathrm{B}(7)-\mathrm{B}(11)-\mathrm{B}(10)$ & 108.1(3) & $\mathrm{C}(2)-\mathrm{C}(4)-\mathrm{H}(4 \mathrm{E})$ & 109.5 \\
\hline $\mathrm{B}(6)-\mathrm{B}(11)-\mathrm{H}(11)$ & 121.7 & $\mathrm{H}(4 \mathrm{C})-\mathrm{C}(4)-\mathrm{H}(4 \mathrm{E})$ & 109.5 \\
\hline $\mathrm{B}(5)-\mathrm{B}(11)-\mathrm{H}(11)$ & 121.8 & $\mathrm{H}(4 \mathrm{D})-\mathrm{C}(4)-\mathrm{H}(4 \mathrm{E})$ & 109.5 \\
\hline $\mathrm{B}(12)-\mathrm{B}(11)-\mathrm{H}(11)$ & 121.9 & $C(6)-C(5)-C(7)$ & $109.3(4)$ \\
\hline $\mathrm{B}(7)-\mathrm{B}(11)-\mathrm{H}(11)$ & 121.7 & $\mathrm{C}(6)-\mathrm{C}(5)-\mathrm{P}(1)$ & $112.9(3)$ \\
\hline $\mathrm{B}(10)-\mathrm{B}(11)-\mathrm{H}(11)$ & 121.7 & $\mathrm{C}(7)-\mathrm{C}(5)-\mathrm{P}(1)$ & $116.4(4)$ \\
\hline $\mathrm{B}(9)-\mathrm{B}(12)-\mathrm{B}(8)$ & $60.1(3)$ & $\mathrm{C}(6)-\mathrm{C}(5)-\mathrm{H}(5 \mathrm{~B})$ & 105.8 \\
\hline $\mathrm{B}(9)-\mathrm{B}(12)-\mathrm{B}(7)$ & 108.1(3) & $\mathrm{C}(7)-\mathrm{C}(5)-\mathrm{H}(5 \mathrm{~B})$ & 105.8 \\
\hline $\mathrm{B}(8)-\mathrm{B}(12)-\mathrm{B}(7)$ & $59.8(3)$ & $\mathrm{P}(1)-\mathrm{C}(5)-\mathrm{H}(5 \mathrm{~B})$ & 105.8 \\
\hline $\mathrm{B}(9)-\mathrm{B}(12)-\mathrm{B}(11)$ & $108.3(3)$ & $\mathrm{C}(5)-\mathrm{C}(6)-\mathrm{H}(6 \mathrm{~B})$ & 109.5 \\
\hline $\mathrm{B}(8)-\mathrm{B}(12)-\mathrm{B}(11)$ & $108.0(3)$ & $\mathrm{C}(5)-\mathrm{C}(6)-\mathrm{H}(6 \mathrm{C})$ & 109.5 \\
\hline $\mathrm{B}(7)-\mathrm{B}(12)-\mathrm{B}(11)$ & $60.1(3)$ & $\mathrm{H}(6 \mathrm{~B})-\mathrm{C}(6)-\mathrm{H}(6 \mathrm{C})$ & 109.5 \\
\hline $\mathrm{B}(9)-\mathrm{B}(12)-\mathrm{B}(10)$ & $60.3(3)$ & $\mathrm{C}(5)-\mathrm{C}(6)-\mathrm{H}(6 \mathrm{D})$ & 109.5 \\
\hline $\mathrm{B}(8)-\mathrm{B}(12)-\mathrm{B}(10)$ & $108.3(3)$ & $\mathrm{H}(6 \mathrm{~B})-\mathrm{C}(6)-\mathrm{H}(6 \mathrm{D})$ & 109.5 \\
\hline $\mathrm{B}(7)-\mathrm{B}(12)-\mathrm{B}(10)$ & 108.2(3) & $\mathrm{H}(6 \mathrm{C})-\mathrm{C}(6)-\mathrm{H}(6 \mathrm{D})$ & 109.5 \\
\hline $\mathrm{B}(11)-\mathrm{B}(12)-\mathrm{B}(10)$ & $60.0(3)$ & $\mathrm{C}(5)-\mathrm{C}(7)-\mathrm{H}(7 \mathrm{C})$ & 109.5 \\
\hline $\mathrm{B}(9)-\mathrm{B}(12)-\mathrm{H}(12)$ & 121.5 & $\mathrm{C}(5)-\mathrm{C}(7)-\mathrm{H}(7 \mathrm{D})$ & 109.5 \\
\hline $\mathrm{B}(8)-\mathrm{B}(12)-\mathrm{H}(12)$ & 121.7 & $\mathrm{H}(7 \mathrm{C})-\mathrm{C}(7)-\mathrm{H}(7 \mathrm{D})$ & 109.5 \\
\hline $\mathrm{B}(7)-\mathrm{B}(12)-\mathrm{H}(12)$ & 121.7 & $\mathrm{C}(5)-\mathrm{C}(7)-\mathrm{H}(7 \mathrm{E})$ & 109.5 \\
\hline $\mathrm{B}(11)-\mathrm{B}(12)-\mathrm{H}(12)$ & 121.7 & H(7C)-C(7)-H(7E) & 109.5 \\
\hline $\mathrm{B}(10)-\mathrm{B}(12)-\mathrm{H}(12)$ & 121.4 & $\mathrm{H}(7 \mathrm{D})-\mathrm{C}(7)-\mathrm{H}(7 \mathrm{E})$ & 109.5 \\
\hline$C(4)-C(2)-C(3)$ & $108.4(3)$ & $\mathrm{C}(4 \mathrm{C})-\mathrm{N}(1 \mathrm{C})-\mathrm{C}(2 \mathrm{C})$ & $110.4(4)$ \\
\hline$C(4)-C(2)-P(1)$ & $119.0(3)$ & $\mathrm{C}(4 \mathrm{C})-\mathrm{N}(1 \mathrm{C})-\mathrm{C}(3 \mathrm{C})$ & $108.5(4)$ \\
\hline $\mathrm{C}(3)-\mathrm{C}(2)-\mathrm{P}(1)$ & $110.0(3)$ & $\mathrm{C}(2 \mathrm{C})-\mathrm{N}(1 \mathrm{C})-\mathrm{C}(3 \mathrm{C})$ & $109.3(4)$ \\
\hline $\mathrm{C}(4)-\mathrm{C}(2)-\mathrm{H}(2 \mathrm{~B})$ & 106.2 & $\mathrm{C}(4 \mathrm{C})-\mathrm{N}(1 \mathrm{C})-\mathrm{C}(1 \mathrm{C})$ & $109.3(3)$ \\
\hline $\mathrm{C}(3)-\mathrm{C}(2)-\mathrm{H}(2 \mathrm{~B})$ & 106.2 & $\mathrm{C}(2 \mathrm{C})-\mathrm{N}(1 \mathrm{C})-\mathrm{C}(1 \mathrm{C})$ & $110.0(3)$ \\
\hline $\mathrm{P}(1)-\mathrm{C}(2)-\mathrm{H}(2 \mathrm{~B})$ & 106.2 & $\mathrm{C}(3 \mathrm{C})-\mathrm{N}(1 \mathrm{C})-\mathrm{C}(1 \mathrm{C})$ & $109.2(3)$ \\
\hline $\mathrm{C}(2)-\mathrm{C}(3)-\mathrm{H}(3 \mathrm{C})$ & 109.5 & $\mathrm{~N}(1 \mathrm{C})-\mathrm{C}(1 \mathrm{C})-\mathrm{H}(1 \mathrm{~B})$ & 109.5 \\
\hline$C(2)-C(3)-H(3 D)$ & 109.5 & $\mathrm{~N}(1 \mathrm{C})-\mathrm{C}(1 \mathrm{C})-\mathrm{H}(1 \mathrm{C})$ & 109.5 \\
\hline $\mathrm{H}(3 \mathrm{C})-\mathrm{C}(3)-\mathrm{H}(3 \mathrm{D})$ & 109.5 & $\mathrm{H}(1 \mathrm{~B})-\mathrm{C}(1 \mathrm{C})-\mathrm{H}(1 \mathrm{C})$ & 109.5 \\
\hline $\mathrm{C}(2)-\mathrm{C}(3)-\mathrm{H}(3 \mathrm{E})$ & 109.5 & $\mathrm{~N}(1 \mathrm{C})-\mathrm{C}(1 \mathrm{C})-\mathrm{H}(1 \mathrm{D})$ & 109.5 \\
\hline $\mathrm{H}(3 \mathrm{C})-\mathrm{C}(3)-\mathrm{H}(3 \mathrm{E})$ & 109.5 & $\mathrm{H}(1 \mathrm{~B})-\mathrm{C}(1 \mathrm{C})-\mathrm{H}(1 \mathrm{D})$ & 109.5 \\
\hline $\mathrm{H}(3 \mathrm{D})-\mathrm{C}(3)-\mathrm{H}(3 \mathrm{E})$ & 109.5 & $\mathrm{H}(1 \mathrm{C})-\mathrm{C}(1 \mathrm{C})-\mathrm{H}(1 \mathrm{D})$ & 109.5 \\
\hline
\end{tabular}




\begin{tabular}{|c|c|c|c|}
\hline $\mathrm{N}(1 \mathrm{C})-\mathrm{C}(2 \mathrm{C})-\mathrm{H}(2 \mathrm{C})$ & 109.5 & $\mathrm{C}(4 \mathrm{~S})-\mathrm{C}(3 \mathrm{~S})-\mathrm{H}(3 \mathrm{~J})$ & 110.5 \\
\hline $\mathrm{N}(1 \mathrm{C})-\mathrm{C}(2 \mathrm{C})-\mathrm{H}(2 \mathrm{D})$ & 109.5 & $\mathrm{C}(2 \mathrm{~S})-\mathrm{C}(3 \mathrm{~S})-\mathrm{H}(3 \mathrm{~J})$ & 110.5 \\
\hline $\mathrm{H}(2 \mathrm{C})-\mathrm{C}(2 \mathrm{C})-\mathrm{H}(2 \mathrm{D})$ & 109.5 & $\mathrm{H}(3 \mathrm{I})-\mathrm{C}(3 \mathrm{~S})-\mathrm{H}(3 \mathrm{~J})$ & 108.7 \\
\hline $\mathrm{N}(1 \mathrm{C})-\mathrm{C}(2 \mathrm{C})-\mathrm{H}(2 \mathrm{E})$ & 109.5 & $\mathrm{O}(1 \mathrm{~S})-\mathrm{C}(4 \mathrm{~S})-\mathrm{C}(3 \mathrm{~S})$ & $107.5(11)$ \\
\hline $\mathrm{H}(2 \mathrm{C})-\mathrm{C}(2 \mathrm{C})-\mathrm{H}(2 \mathrm{E})$ & 109.5 & $\mathrm{O}(1 \mathrm{~S})-\mathrm{C}(4 \mathrm{~S})-\mathrm{H}(4 \mathrm{I})$ & 110.2 \\
\hline $\mathrm{H}(2 \mathrm{D})-\mathrm{C}(2 \mathrm{C})-\mathrm{H}(2 \mathrm{E})$ & 109.5 & $\mathrm{C}(3 \mathrm{~S})-\mathrm{C}(4 \mathrm{~S})-\mathrm{H}(4 \mathrm{I})$ & 110.2 \\
\hline $\mathrm{N}(1 \mathrm{C})-\mathrm{C}(3 \mathrm{C})-\mathrm{H}(3 \mathrm{~F})$ & 109.5 & $\mathrm{O}(1 \mathrm{~S})-\mathrm{C}(4 \mathrm{~S})-\mathrm{H}(4 \mathrm{~J})$ & 110.2 \\
\hline $\mathrm{N}(1 \mathrm{C})-\mathrm{C}(3 \mathrm{C})-\mathrm{H}(3 \mathrm{G})$ & 109.5 & $\mathrm{C}(3 \mathrm{~S})-\mathrm{C}(4 \mathrm{~S})-\mathrm{H}(4 \mathrm{~J})$ & 110.2 \\
\hline $\mathrm{H}(3 \mathrm{~F})-\mathrm{C}(3 \mathrm{C})-\mathrm{H}(3 \mathrm{G})$ & 109.5 & $\mathrm{H}(4 \mathrm{I})-\mathrm{C}(4 \mathrm{~S})-\mathrm{H}(4 \mathrm{~J})$ & 108.5 \\
\hline $\mathrm{N}(1 \mathrm{C})-\mathrm{C}(3 \mathrm{C})-\mathrm{H}(3 \mathrm{H})$ & 109.5 & $\mathrm{C}(1 \mathrm{D})-\mathrm{O}(1 \mathrm{D})-\mathrm{C}(4 \mathrm{D})$ & $104.0(17)$ \\
\hline $\mathrm{H}(3 \mathrm{~F})-\mathrm{C}(3 \mathrm{C})-\mathrm{H}(3 \mathrm{H})$ & 109.5 & $\mathrm{O}(1 \mathrm{D})-\mathrm{C}(1 \mathrm{D})-\mathrm{C}(2 \mathrm{D})$ & $109.3(17)$ \\
\hline $\mathrm{H}(3 \mathrm{G})-\mathrm{C}(3 \mathrm{C})-\mathrm{H}(3 \mathrm{H})$ & 109.5 & $\mathrm{O}(1 \mathrm{D})-\mathrm{C}(1 \mathrm{D})-\mathrm{H}(1 \mathrm{G})$ & 109.8 \\
\hline $\mathrm{N}(1 \mathrm{C})-\mathrm{C}(4 \mathrm{C})-\mathrm{H}(4 \mathrm{~F})$ & 109.5 & $\mathrm{C}(2 \mathrm{D})-\mathrm{C}(1 \mathrm{D})-\mathrm{H}(1 \mathrm{G})$ & 109.8 \\
\hline $\mathrm{N}(1 \mathrm{C})-\mathrm{C}(4 \mathrm{C})-\mathrm{H}(4 \mathrm{G})$ & 109.5 & $\mathrm{O}(1 \mathrm{D})-\mathrm{C}(1 \mathrm{D})-\mathrm{H}(1 \mathrm{H})$ & 109.8 \\
\hline $\mathrm{H}(4 \mathrm{~F})-\mathrm{C}(4 \mathrm{C})-\mathrm{H}(4 \mathrm{G})$ & 109.5 & $\mathrm{C}(2 \mathrm{D})-\mathrm{C}(1 \mathrm{D})-\mathrm{H}(1 \mathrm{H})$ & 109.8 \\
\hline $\mathrm{N}(1 \mathrm{C})-\mathrm{C}(4 \mathrm{C})-\mathrm{H}(4 \mathrm{H})$ & 109.5 & $\mathrm{H}(1 \mathrm{G})-\mathrm{C}(1 \mathrm{D})-\mathrm{H}(1 \mathrm{H})$ & 108.3 \\
\hline $\mathrm{H}(4 \mathrm{~F})-\mathrm{C}(4 \mathrm{C})-\mathrm{H}(4 \mathrm{H})$ & 109.5 & $\mathrm{C}(3 \mathrm{D})-\mathrm{C}(2 \mathrm{D})-\mathrm{C}(1 \mathrm{D})$ & $105.5(15)$ \\
\hline $\mathrm{H}(4 \mathrm{G})-\mathrm{C}(4 \mathrm{C})-\mathrm{H}(4 \mathrm{H})$ & 109.5 & $\mathrm{C}(3 \mathrm{D})-\mathrm{C}(2 \mathrm{D})-\mathrm{H}(2 \mathrm{H})$ & 110.6 \\
\hline $\mathrm{C}(1 \mathrm{~S})-\mathrm{O}(1 \mathrm{~S})-\mathrm{C}(4 \mathrm{~S})$ & $108.1(11)$ & $\mathrm{C}(1 \mathrm{D})-\mathrm{C}(2 \mathrm{D})-\mathrm{H}(2 \mathrm{H})$ & 110.6 \\
\hline $\mathrm{O}(1 \mathrm{~S})-\mathrm{C}(1 \mathrm{~S})-\mathrm{C}(2 \mathrm{~S})$ & $105.8(11)$ & $\mathrm{C}(3 \mathrm{D})-\mathrm{C}(2 \mathrm{D})-\mathrm{H}(2 \mathrm{I})$ & 110.6 \\
\hline $\mathrm{O}(1 \mathrm{~S})-\mathrm{C}(1 \mathrm{~S})-\mathrm{H}(1 \mathrm{E})$ & 110.6 & $\mathrm{C}(1 \mathrm{D})-\mathrm{C}(2 \mathrm{D})-\mathrm{H}(2 \mathrm{I})$ & 110.6 \\
\hline $\mathrm{C}(2 \mathrm{~S})-\mathrm{C}(1 \mathrm{~S})-\mathrm{H}(1 \mathrm{E})$ & 110.6 & $\mathrm{H}(2 \mathrm{H})-\mathrm{C}(2 \mathrm{D})-\mathrm{H}(2 \mathrm{I})$ & 108.8 \\
\hline $\mathrm{O}(1 \mathrm{~S})-\mathrm{C}(1 \mathrm{~S})-\mathrm{H}(1 \mathrm{~F})$ & 110.6 & $\mathrm{C}(2 \mathrm{D})-\mathrm{C}(3 \mathrm{D})-\mathrm{C}(4 \mathrm{D})$ & $106.5(14)$ \\
\hline $\mathrm{C}(2 \mathrm{~S})-\mathrm{C}(1 \mathrm{~S})-\mathrm{H}(1 \mathrm{~F})$ & 110.6 & $\mathrm{C}(2 \mathrm{D})-\mathrm{C}(3 \mathrm{D})-\mathrm{H}(3 \mathrm{~K})$ & 110.4 \\
\hline $\mathrm{H}(1 \mathrm{E})-\mathrm{C}(1 \mathrm{~S})-\mathrm{H}(1 \mathrm{~F})$ & 108.7 & $\mathrm{C}(4 \mathrm{D})-\mathrm{C}(3 \mathrm{D})-\mathrm{H}(3 \mathrm{~K})$ & 110.4 \\
\hline$C(3 S)-C(2 S)-C(1 S)$ & $105.3(9)$ & $\mathrm{C}(2 \mathrm{D})-\mathrm{C}(3 \mathrm{D})-\mathrm{H}(3 \mathrm{~L})$ & 110.4 \\
\hline $\mathrm{C}(3 \mathrm{~S})-\mathrm{C}(2 \mathrm{~S})-\mathrm{H}(2 \mathrm{~F})$ & 110.7 & $\mathrm{C}(4 \mathrm{D})-\mathrm{C}(3 \mathrm{D})-\mathrm{H}(3 \mathrm{~L})$ & 110.4 \\
\hline $\mathrm{C}(1 \mathrm{~S})-\mathrm{C}(2 \mathrm{~S})-\mathrm{H}(2 \mathrm{~F})$ & 110.7 & $\mathrm{H}(3 \mathrm{~K})-\mathrm{C}(3 \mathrm{D})-\mathrm{H}(3 \mathrm{~L})$ & 108.6 \\
\hline $\mathrm{C}(3 \mathrm{~S})-\mathrm{C}(2 \mathrm{~S})-\mathrm{H}(2 \mathrm{G})$ & 110.7 & $\mathrm{O}(1 \mathrm{D})-\mathrm{C}(4 \mathrm{D})-\mathrm{C}(3 \mathrm{D})$ & $108.0(18)$ \\
\hline $\mathrm{C}(1 \mathrm{~S})-\mathrm{C}(2 \mathrm{~S})-\mathrm{H}(2 \mathrm{G})$ & 110.7 & $\mathrm{O}(1 \mathrm{D})-\mathrm{C}(4 \mathrm{D})-\mathrm{H}(4 \mathrm{~K})$ & 110.1 \\
\hline $\mathrm{H}(2 \mathrm{~F})-\mathrm{C}(2 \mathrm{~S})-\mathrm{H}(2 \mathrm{G})$ & 108.8 & $\mathrm{C}(3 \mathrm{D})-\mathrm{C}(4 \mathrm{D})-\mathrm{H}(4 \mathrm{~K})$ & 110.1 \\
\hline$C(4 S)-C(3 S)-C(2 S)$ & 106.2(9) & $\mathrm{O}(1 \mathrm{D})-\mathrm{C}(4 \mathrm{D})-\mathrm{H}(4 \mathrm{~L})$ & 110.1 \\
\hline $\mathrm{C}(4 \mathrm{~S})-\mathrm{C}(3 \mathrm{~S})-\mathrm{H}(3 \mathrm{I})$ & 110.5 & $\mathrm{C}(3 \mathrm{D})-\mathrm{C}(4 \mathrm{D})-\mathrm{H}(4 \mathrm{~L})$ & 110.1 \\
\hline $\mathrm{C}(2 \mathrm{~S})-\mathrm{C}(3 \mathrm{~S})-\mathrm{H}(3 \mathrm{I})$ & 110.5 & $\mathrm{H}(4 \mathrm{~K})-\mathrm{C}(4 \mathrm{D})-\mathrm{H}(4 \mathrm{~L})$ & 108.4 \\
\hline
\end{tabular}

Symmetry transformations used to generate equivalent atoms: 
Table 10. Anisotropic displacement parameters $\left(\AA^{2} \times 10^{3}\right)$ for vL147JE_0m. The anisotropic displacement factor exponent takes the form: $-2 \pi^{2}\left[h^{2} a^{* 2} U^{11}+\ldots+2 h k a^{*} b^{*} U^{12}\right]$

\begin{tabular}{|c|c|c|c|c|c|c|}
\hline & $\mathrm{U}^{11}$ & $\mathrm{U}^{22}$ & $\mathrm{U}^{33}$ & $\mathrm{U}^{23}$ & $\mathrm{U}^{13}$ & $\mathrm{U}^{12}$ \\
\hline $\operatorname{Ir}(1)$ & $8(1)$ & $11(1)$ & 11(1) & $0(1)$ & $-1(1)$ & $0(1)$ \\
\hline $\mathrm{Cl}(1)$ & $15(1)$ & $19(1)$ & $15(1)$ & $-3(1)$ & $-2(1)$ & $-1(1)$ \\
\hline $\mathrm{C}(1 \mathrm{~A})$ & $9(2)$ & $25(2)$ & $17(2)$ & $-1(2)$ & $-2(1)$ & $-5(1)$ \\
\hline$C(2 A)$ & $10(2)$ & $22(2)$ & $15(2)$ & $1(2)$ & $-2(1)$ & $1(1)$ \\
\hline $\mathrm{C}(3 \mathrm{~A})$ & $13(2)$ & $20(2)$ & $24(2)$ & $0(1)$ & $0(1)$ & $5(1)$ \\
\hline $\mathrm{C}(4 \mathrm{~A})$ & $15(2)$ & $16(2)$ & $30(2)$ & $-3(1)$ & $0(1)$ & $3(1)$ \\
\hline $\mathrm{C}(5 \mathrm{~A})$ & 11(1) & $17(2)$ & $16(2)$ & $-4(1)$ & $0(1)$ & $1(1)$ \\
\hline $\mathrm{C}(6 \mathrm{~A})$ & 11(1) & $25(2)$ & $13(1)$ & $-1(1)$ & $0(1)$ & 1(1) \\
\hline $\mathrm{C}(7 \mathrm{~A})$ & $17(1)$ & $27(2)$ & $14(1)$ & $4(2)$ & $2(1)$ & $1(2)$ \\
\hline $\mathrm{C}(8 \mathrm{~A})$ & $15(2)$ & $26(2)$ & $23(2)$ & $6(2)$ & $1(1)$ & $-6(1)$ \\
\hline $\mathrm{P}(1)$ & $9(1)$ & $10(1)$ & 11(1) & $1(1)$ & $-1(1)$ & $0(1)$ \\
\hline $\mathrm{C}(1)$ & $8(2)$ & $13(2)$ & $12(2)$ & $1(1)$ & $-1(1)$ & $-1(1)$ \\
\hline $\mathrm{B}(2)$ & $16(2)$ & $22(2)$ & $11(2)$ & $2(1)$ & $-2(1)$ & $-4(1)$ \\
\hline $\mathrm{B}(3)$ & $11(2)$ & $17(2)$ & $16(2)$ & $4(1)$ & $-5(1)$ & $-1(1)$ \\
\hline $\mathrm{B}(4)$ & $16(2)$ & $12(2)$ & $18(2)$ & $2(1)$ & $-3(2)$ & $-2(1)$ \\
\hline $\mathrm{B}(5)$ & $12(2)$ & $19(2)$ & $16(2)$ & $5(2)$ & $-3(1)$ & $-3(2)$ \\
\hline $\mathrm{B}(6)$ & $11(2)$ & $25(2)$ & $16(2)$ & $3(2)$ & 1(1) & $-2(1)$ \\
\hline $\mathrm{B}(7)$ & $16(2)$ & $35(2)$ & $14(2)$ & $9(2)$ & $0(1)$ & $-7(2)$ \\
\hline $\mathrm{B}(8)$ & $14(2)$ & $26(2)$ & $19(2)$ & $9(2)$ & $-4(1)$ & $-6(1)$ \\
\hline $\mathrm{B}(9)$ & $17(2)$ & $17(2)$ & $27(2)$ & $10(2)$ & $-6(2)$ & $-4(1)$ \\
\hline $\mathrm{B}(10)$ & $18(2)$ & $18(2)$ & $22(2)$ & $6(2)$ & $-6(2)$ & $-6(1)$ \\
\hline $\mathrm{B}(11)$ & $13(2)$ & $29(2)$ & $18(2)$ & $10(2)$ & $-4(1)$ & $-6(2)$ \\
\hline $\mathrm{B}(12)$ & $17(2)$ & $28(2)$ & $24(2)$ & $12(2)$ & $-8(2)$ & $-9(2)$ \\
\hline $\mathrm{C}(2)$ & $10(1)$ & $17(1)$ & $14(1)$ & $2(2)$ & $-3(1)$ & $2(2)$ \\
\hline $\mathrm{C}(3)$ & $22(2)$ & $19(2)$ & $17(2)$ & $9(2)$ & $-3(2)$ & $-1(2)$ \\
\hline $\mathrm{C}(4)$ & $15(2)$ & $19(2)$ & $17(2)$ & $1(1)$ & $-5(2)$ & $-1(1)$ \\
\hline $\mathrm{C}(5)$ & $22(2)$ & $16(2)$ & $19(2)$ & $-1(2)$ & $1(2)$ & $7(2)$ \\
\hline $\mathrm{C}(6)$ & $32(2)$ & $12(2)$ & $31(2)$ & $-1(1)$ & $-8(2)$ & $2(2)$ \\
\hline $\mathrm{C}(7)$ & $22(2)$ & $29(3)$ & $33(2)$ & $0(2)$ & $6(2)$ & $15(2)$ \\
\hline $\mathrm{N}(1 \mathrm{C})$ & $14(1)$ & $22(2)$ & $16(1)$ & $1(1)$ & $2(1)$ & $3(1)$ \\
\hline
\end{tabular}




$\begin{array}{lllllll}\mathrm{C}(1 \mathrm{C}) & 19(2) & 24(2) & 18(2) & -1(1) & -1(1) & 6(1) \\ \mathrm{C}(2 \mathrm{C}) & 22(2) & 56(3) & 41(3) & -31(2) & -2(2) & -2(2) \\ \mathrm{C}(3 \mathrm{C}) & 26(2) & 26(2) & 32(2) & 8(2) & -5(2) & 7(2) \\ \mathrm{C}(4 \mathrm{C}) & 44(3) & 39(3) & 34(3) & 14(2) & 22(2) & 9(2) \\ \mathrm{O}(1 \mathrm{~S}) & 27(5) & 17(4) & 44(5) & -6(3) & 5(4) & -3(4) \\ \mathrm{C}(1 \mathrm{~S}) & 37(7) & 72(6) & 41(4) & 12(5) & 8(4) & 0(4) \\ \mathrm{C}(2 \mathrm{~S}) & 94(9) & 59(6) & 29(4) & -13(4) & 30(4) & -43(5) \\ \mathrm{C}(3 \mathrm{~S}) & 33(4) & 47(4) & 100(6) & -24(4) & -13(4) & 14(3) \\ \mathrm{C}(4 S) & 33(4) & 47(4) & 100(6) & -24(4) & -13(4) & 14(3) \\ \mathrm{O}(1 \mathrm{D}) & 34(10) & 19(9) & 49(9) & -15(7) & 11(8) & -2(10) \\ \mathrm{C}(1 \mathrm{D}) & 49(8) & 40(6) & 38(5) & -8(4) & 12(5) & -3(5) \\ \mathrm{C}(2 \mathrm{D}) & 33(4) & 47(4) & 100(6) & -24(4) & -13(4) & 14(3) \\ \mathrm{C}(3 \mathrm{D}) & 49(8) & 40(6) & 38(5) & -8(4) & 12(5) & -3(5) \\ \mathrm{C}(4 \mathrm{D}) & 37(7) & 72(6) & 41(4) & 12(5) & 8(4) & 0(4)\end{array}$


Table 11. Hydrogen coordinates ( $\times 10^{4}$ ) and isotropic displacement parameters $\left(\AA^{2} \times 10^{3}\right)$ for vL147JE_0m.

\begin{tabular}{|c|c|c|c|c|}
\hline & $\mathrm{x}$ & $\mathrm{y}$ & $\mathrm{z}$ & $\mathrm{U}(\mathrm{eq})$ \\
\hline $\mathrm{H}(1 \mathrm{~A})$ & $3300(50)$ & $1550(40)$ & $3070(30)$ & 20 \\
\hline $\mathrm{H}(2 \mathrm{~A})$ & $3340(50)$ & $3510(50)$ & $2500(20)$ & 19 \\
\hline $\mathrm{H}(3 \mathrm{~A})$ & 2563 & 4252 & 4170 & 23 \\
\hline $\mathrm{H}(3 \mathrm{~B})$ & 2669 & 5086 & 3290 & 23 \\
\hline $\mathrm{H}(4 \mathrm{~A})$ & 4680 & 5698 & 3678 & 25 \\
\hline $\mathrm{H}(4 \mathrm{~B})$ & 4155 & 5490 & 4669 & 25 \\
\hline $\mathrm{H}(5 \mathrm{~A})$ & $6240(30)$ & $4540(40)$ & $4350(30)$ & 17 \\
\hline $\mathrm{H}(6 \mathrm{~A})$ & $5970(30)$ & $2800(70)$ & $5120(20)$ & 20 \\
\hline $\mathrm{H}(7 \mathrm{~A})$ & 3448 & 3390 & 5245 & 23 \\
\hline $\mathrm{H}(7 \mathrm{~B})$ & 4113 & 2208 & 5685 & 23 \\
\hline $\mathrm{H}(8 \mathrm{~A})$ & 3452 & 1009 & 4532 & 26 \\
\hline $\mathrm{H}(8 \mathrm{~B})$ & 2275 & 1946 & 4528 & 26 \\
\hline $\mathrm{H}(2)$ & 7482 & 3291 & 1136 & 20 \\
\hline $\mathrm{H}(3)$ & 6027 & 4880 & 2329 & 18 \\
\hline $\mathrm{H}(4)$ & 7792 & 5691 & 3812 & 18 \\
\hline $\mathrm{H}(5)$ & 10365 & 4606 & 3537 & 19 \\
\hline $\mathrm{H}(6)$ & 10168 & 3137 & 1866 & 21 \\
\hline $\mathrm{H}(7)$ & 9524 & 4767 & 325 & 26 \\
\hline $\mathrm{H}(8)$ & 6973 & 5844 & 617 & 24 \\
\hline $\mathrm{H}(9)$ & 7171 & 7327 & 2282 & 25 \\
\hline $\mathrm{H}(10)$ & 9866 & 7157 & 3040 & 24 \\
\hline $\mathrm{H}(11)$ & 11320 & 5576 & 1819 & 24 \\
\hline $\mathrm{H}(12)$ & 9335 & 7262 & 1051 & 28 \\
\hline $\mathrm{H}(2 \mathrm{~B})$ & 9689 & 2722 & 4230 & 17 \\
\hline $\mathrm{H}(3 \mathrm{C})$ & 7676 & 1593 & 5184 & 29 \\
\hline $\mathrm{H}(3 \mathrm{D})$ & 9179 & 1545 & 5473 & 29 \\
\hline $\mathrm{H}(3 \mathrm{E})$ & 8658 & 885 & 4566 & 29 \\
\hline $\mathrm{H}(4 \mathrm{C})$ & 7811 & 3920 & 5264 & 26 \\
\hline $\mathrm{H}(4 \mathrm{D})$ & 8950 & 4576 & 4754 & 26 \\
\hline $\mathrm{H}(4 \mathrm{E})$ & 9281 & 3703 & 5595 & 26 \\
\hline $\mathrm{H}(5 \mathrm{~B})$ & 8222 & 1538 & 2105 & 23 \\
\hline
\end{tabular}




\begin{tabular}{|c|c|c|c|c|}
\hline $\mathrm{H}(6 \mathrm{~B})$ & 7845 & -421 & 2563 & 38 \\
\hline $\mathrm{H}(6 \mathrm{C})$ & 6659 & 399 & 2852 & 38 \\
\hline $\mathrm{H}(6 \mathrm{D})$ & 7739 & -7 & 3595 & 38 \\
\hline $\mathrm{H}(7 \mathrm{C})$ & 9970 & 742 & 3514 & 42 \\
\hline $\mathrm{H}(7 \mathrm{D})$ & 10304 & 1860 & 2876 & 42 \\
\hline $\mathrm{H}(7 \mathrm{E})$ & 10085 & 542 & 2448 & 42 \\
\hline $\mathrm{H}(1 \mathrm{~B})$ & 8034 & 8683 & 9032 & 30 \\
\hline $\mathrm{H}(1 \mathrm{C})$ & 6877 & 7751 & 9169 & 30 \\
\hline H(1D) & 7997 & 7895 & 9939 & 30 \\
\hline $\mathrm{H}(2 \mathrm{C})$ & 8168 & 10540 & 9915 & 59 \\
\hline $\mathrm{H}(2 \mathrm{D})$ & 8137 & 9778 & 10834 & 59 \\
\hline $\mathrm{H}(2 \mathrm{E})$ & 7105 & 10833 & 10638 & 59 \\
\hline $\mathrm{H}(3 \mathrm{~F})$ & 5383 & 10558 & 9491 & 43 \\
\hline $\mathrm{H}(3 \mathrm{G})$ & 5321 & 9345 & 8899 & 43 \\
\hline $\mathrm{H}(3 \mathrm{H})$ & 6452 & 10306 & 8763 & 43 \\
\hline $\mathrm{H}(4 \mathrm{~F})$ & 6379 & 8323 & 11046 & 58 \\
\hline $\mathrm{H}(4 \mathrm{G})$ & 5265 & 8178 & 10272 & 58 \\
\hline $\mathrm{H}(4 \mathrm{H})$ & 5356 & 9389 & 10865 & 58 \\
\hline $\mathrm{H}(1 \mathrm{E})$ & 4740 & 7446 & 2453 & 60 \\
\hline $\mathrm{H}(1 \mathrm{~F})$ & 3296 & 7108 & 2722 & 60 \\
\hline $\mathrm{H}(2 \mathrm{~F})$ & 3224 & 9015 & 3193 & 72 \\
\hline $\mathrm{H}(2 \mathrm{G})$ & 4385 & 9423 & 2570 & 72 \\
\hline $\mathrm{H}(3 \mathrm{I})$ & 1786 & 9558 & 2152 & 73 \\
\hline $\mathrm{H}(3 \mathrm{~J})$ & 2937 & 10139 & 1598 & 73 \\
\hline $\mathrm{H}(4 \mathrm{I})$ & 1615 & 8047 & 1221 & 73 \\
\hline $\mathrm{H}(4 \mathrm{~J})$ & 2600 & 8740 & 589 & 73 \\
\hline $\mathrm{H}(1 \mathrm{G})$ & 1916 & 7593 & 962 & 51 \\
\hline $\mathrm{H}(1 \mathrm{H})$ & 2835 & 8555 & 491 & 51 \\
\hline $\mathrm{H}(2 \mathrm{H})$ & 2379 & 9974 & 1436 & 73 \\
\hline $\mathrm{H}(2 \mathrm{I})$ & 1138 & 9154 & 1632 & 73 \\
\hline $\mathrm{H}(3 \mathrm{~K})$ & 1909 & 8736 & 2951 & 51 \\
\hline $\mathrm{H}(3 \mathrm{~L})$ & 3091 & 9639 & 2783 & 51 \\
\hline $\mathrm{H}(4 \mathrm{~K})$ & 4319 & 8047 & 2790 & 60 \\
\hline $\mathrm{H}(4 \mathrm{~L})$ & 3097 & 7153 & 2734 & 60 \\
\hline
\end{tabular}


Table 12. Torsion angles $\left[^{\circ}\right]$ for vL147JE_0m.

\begin{tabular}{lc}
\hline $\mathrm{C}(8 \mathrm{~A})-\mathrm{C}(1 \mathrm{~A})-\mathrm{C}(2 \mathrm{~A})-\mathrm{C}(3 \mathrm{~A})$ & $-1.8(7)$ \\
$\operatorname{Ir}(1)-\mathrm{C}(1 \mathrm{~A})-\mathrm{C}(2 \mathrm{~A})-\mathrm{C}(3 \mathrm{~A})$ & $-104.8(4)$ \\
$\mathrm{C}(8 \mathrm{~A})-\mathrm{C}(1 \mathrm{~A})-\mathrm{C}(2 \mathrm{~A})-\mathrm{Ir}(1)$ & $103.0(4)$ \\
$\mathrm{C}(1 \mathrm{~A})-\mathrm{C}(2 \mathrm{~A})-\mathrm{C}(3 \mathrm{~A})-\mathrm{C}(4 \mathrm{~A})$ & $93.5(5)$ \\
$\operatorname{Ir}(1)-\mathrm{C}(2 \mathrm{~A})-\mathrm{C}(3 \mathrm{~A})-\mathrm{C}(4 \mathrm{~A})$ & $12.0(4)$ \\
$\mathrm{C}(2 \mathrm{~A})-\mathrm{C}(3 \mathrm{~A})-\mathrm{C}(4 \mathrm{~A})-\mathrm{C}(5 \mathrm{~A})$ & $-30.3(5)$ \\
$\mathrm{C}(3 \mathrm{~A})-\mathrm{C}(4 \mathrm{~A})-\mathrm{C}(5 \mathrm{~A})-\mathrm{C}(6 \mathrm{~A})$ & $-47.5(5)$ \\
$\mathrm{C}(3 \mathrm{~A})-\mathrm{C}(4 \mathrm{~A})-\mathrm{C}(5 \mathrm{~A})-\mathrm{Ir}(1)$ & $34.9(4)$ \\
$\mathrm{C}(4 \mathrm{~A})-\mathrm{C}(5 \mathrm{~A})-\mathrm{C}(6 \mathrm{~A})-\mathrm{C}(7 \mathrm{~A})$ & $-1.9(5)$ \\
$\operatorname{Ir}(1)-\mathrm{C}(5 \mathrm{~A})-\mathrm{C}(6 \mathrm{~A})-\mathrm{C}(7 \mathrm{~A})$ & $-106.9(3)$ \\
$\mathrm{C}(4 \mathrm{~A})-\mathrm{C}(5 \mathrm{~A})-\mathrm{C}(6 \mathrm{~A})-\mathrm{Ir}(1)$ & $104.9(3)$ \\
$\mathrm{C}(5 \mathrm{~A})-\mathrm{C}(6 \mathrm{~A})-\mathrm{C}(7 \mathrm{~A})-\mathrm{C}(8 \mathrm{~A})$ & $94.7(5)$ \\
$\operatorname{Ir}(1)-\mathrm{C}(6 \mathrm{~A})-\mathrm{C}(7 \mathrm{~A})-\mathrm{C}(8 \mathrm{~A})$ & $14.7(5)$ \\
$\mathrm{C}(2 \mathrm{~A})-\mathrm{C}(1 \mathrm{~A})-\mathrm{C}(8 \mathrm{~A})-\mathrm{C}(7 \mathrm{~A})$ & $-47.0(6)$ \\
$\operatorname{Ir}(1)-\mathrm{C}(1 \mathrm{~A})-\mathrm{C}(8 \mathrm{~A})-\mathrm{C}(7 \mathrm{~A})$ & $34.7(4)$ \\
$\mathrm{C}(6 \mathrm{~A})-\mathrm{C}(7 \mathrm{~A})-\mathrm{C}(8 \mathrm{~A})-\mathrm{C}(1 \mathrm{~A})$ & $-32.4(5)$ \\
$\mathrm{C}(5)-\mathrm{P}(1)-\mathrm{C}(1)-\mathrm{B}(5)$ & $-95.7(4)$ \\
$\mathrm{C}(2)-\mathrm{P}(1)-\mathrm{C}(1)-\mathrm{B}(5)$ & $11.0(4)$ \\
$\operatorname{Ir}(1)-\mathrm{P}(1)-\mathrm{C}(1)-\mathrm{B}(5)$ & $144.7(3)$ \\
$\mathrm{C}(5)-\mathrm{P}(1)-\mathrm{C}(1)-\mathrm{B}(6)$ & $-20.9(3)$ \\
$\mathrm{C}(2)-\mathrm{P}(1)-\mathrm{C}(1)-\mathrm{B}(6)$ & $85.9(3)$ \\
$\operatorname{Ir}(1)-\mathrm{P}(1)-\mathrm{C}(1)-\mathrm{B}(6)$ & $-140.4(3)$ \\
$\mathrm{C}(5)-\mathrm{P}(1)-\mathrm{C}(1)-\mathrm{B}(2)$ & $52.0(3)$ \\
$\mathrm{C}(2)-\mathrm{P}(1)-\mathrm{C}(1)-\mathrm{B}(2)$ & $158.7(3)$ \\
$\operatorname{Ir}(1)-\mathrm{P}(1)-\mathrm{C}(1)-\mathrm{B}(2)$ & $-67.6(3)$ \\
$\mathrm{C}(5)-\mathrm{P}(1)-\mathrm{C}(1)-\mathrm{B}(4)$ & $-168.6(3)$ \\
$\mathrm{C}(2)-\mathrm{P}(1)-\mathrm{C}(1)-\mathrm{B}(4)$ & $-61.9(3)$ \\
$\operatorname{Ir}(1)-\mathrm{P}(1)-\mathrm{C}(1)-\mathrm{B}(4)$ & $71.8(3)$ \\
$\mathrm{C}(5)-\mathrm{P}(1)-\mathrm{C}(1)-\mathrm{B}(3)$ & $121.9(3)$ \\
$\mathrm{C}(2)-\mathrm{P}(1)-\mathrm{C}(1)-\mathrm{B}(3)$ & $-131.3(3)$ \\
$\operatorname{Ir}(1)-\mathrm{P}(1)-\mathrm{C}(1)-\mathrm{B}(3)$ & $2.3(3)$ \\
$\mathrm{B}(5)-\mathrm{C}(1)-\mathrm{B}(2)-\mathrm{B}(7)$ & $-2.2(4)$ \\
$\mathrm{B}(6)-\mathrm{C}(1)-\mathrm{B}(2)-\mathrm{B}(7)$ & $-38.9(3)$ \\
$\mathrm{B}(4)-\mathrm{C}(1)-\mathrm{B}(2)-\mathrm{B}(7)$ & \\
& \\
& \\
&
\end{tabular}


$\mathrm{B}(3)-\mathrm{C}(1)-\mathrm{B}(2)-\mathrm{B}(7)$

$\mathrm{P}(1)-\mathrm{C}(1)-\mathrm{B}(2)-\mathrm{B}(7)$

$\mathrm{B}(5)-\mathrm{C}(1)-\mathrm{B}(2)-\mathrm{B}(3)$

$\mathrm{B}(6)-\mathrm{C}(1)-\mathrm{B}(2)-\mathrm{B}(3)$

$\mathrm{B}(4)-\mathrm{C}(1)-\mathrm{B}(2)-\mathrm{B}(3)$

$\mathrm{P}(1)-\mathrm{C}(1)-\mathrm{B}(2)-\mathrm{B}(3)$

$\mathrm{B}(5)-\mathrm{C}(1)-\mathrm{B}(2)-\mathrm{B}(8)$

$\mathrm{B}(6)-\mathrm{C}(1)-\mathrm{B}(2)-\mathrm{B}(8)$

$\mathrm{B}(4)-\mathrm{C}(1)-\mathrm{B}(2)-\mathrm{B}(8)$

$\mathrm{B}(3)-\mathrm{C}(1)-\mathrm{B}(2)-\mathrm{B}(8)$

$\mathrm{P}(1)-\mathrm{C}(1)-\mathrm{B}(2)-\mathrm{B}(8)$

$\mathrm{B}(5)-\mathrm{C}(1)-\mathrm{B}(2)-\mathrm{B}(6)$

$\mathrm{B}(4)-\mathrm{C}(1)-\mathrm{B}(2)-\mathrm{B}(6)$

$\mathrm{B}(3)-\mathrm{C}(1)-\mathrm{B}(2)-\mathrm{B}(6)$

$\mathrm{P}(1)-\mathrm{C}(1)-\mathrm{B}(2)-\mathrm{B}(6)$

$\mathrm{B}(5)-\mathrm{C}(1)-\mathrm{B}(3)-\mathrm{B}(8)$

$\mathrm{B}(6)-\mathrm{C}(1)-\mathrm{B}(3)-\mathrm{B}(8)$

$\mathrm{B}(2)-\mathrm{C}(1)-\mathrm{B}(3)-\mathrm{B}(8)$

$\mathrm{B}(4)-\mathrm{C}(1)-\mathrm{B}(3)-\mathrm{B}(8)$

$\mathrm{P}(1)-\mathrm{C}(1)-\mathrm{B}(3)-\mathrm{B}(8)$

$\mathrm{B}(5)-\mathrm{C}(1)-\mathrm{B}(3)-\mathrm{B}(4)$

$\mathrm{B}(6)-\mathrm{C}(1)-\mathrm{B}(3)-\mathrm{B}(4)$

$\mathrm{B}(2)-\mathrm{C}(1)-\mathrm{B}(3)-\mathrm{B}(4)$

$\mathrm{P}(1)-\mathrm{C}(1)-\mathrm{B}(3)-\mathrm{B}(4)$

$\mathrm{B}(5)-\mathrm{C}(1)-\mathrm{B}(3)-\mathrm{B}(9)$

$\mathrm{B}(6)-\mathrm{C}(1)-\mathrm{B}(3)-\mathrm{B}(9)$

$\mathrm{B}(2)-\mathrm{C}(1)-\mathrm{B}(3)-\mathrm{B}(9)$

$\mathrm{B}(4)-\mathrm{C}(1)-\mathrm{B}(3)-\mathrm{B}(9)$

$\mathrm{P}(1)-\mathrm{C}(1)-\mathrm{B}(3)-\mathrm{B}(9)$

$\mathrm{B}(5)-\mathrm{C}(1)-\mathrm{B}(3)-\mathrm{B}(2)$

$\mathrm{B}(6)-\mathrm{C}(1)-\mathrm{B}(3)-\mathrm{B}(2)$

$\mathrm{B}(4)-\mathrm{C}(1)-\mathrm{B}(3)-\mathrm{B}(2)$

$\mathrm{P}(1)-\mathrm{C}(1)-\mathrm{B}(3)-\mathrm{B}(2)$

$\mathrm{B}(7)-\mathrm{B}(2)-\mathrm{B}(3)-\mathrm{C}(1)$

$\mathrm{B}(8)-\mathrm{B}(2)-\mathrm{B}(3)-\mathrm{C}(1)$

$\mathrm{B}(6)-\mathrm{B}(2)-\mathrm{B}(3)-\mathrm{C}(1)$
101.7(3)

$-152.7(3)$

$-103.9(3)$

$-140.6(3)$

$-36.2(3)$

105.6(3)

$-64.8(4)$

$-101.6(3)$

2.9(4)

39.1(3)

144.7(3)

36.8(3)

104.5(3)

140.6(3)

$-113.8(3)$

65.1(4)

$-2.3(4)$

$-39.3(3)$

101.9(3)

$-149.0(3)$

$-36.9(3)$

$-104.2(3)$

$-141.2(3)$

109.1(3)

2.1(4)

$-65.3(4)$

$-102.3(3)$

39.0(3)

148.1(3)

104.4(4)

$37.0(3)$

141.2(3)

$-109.7(3)$

$-98.3(3)$

$-135.4(3)$

$-34.9(3)$ 
$\mathrm{C}(1)-\mathrm{B}(2)-\mathrm{B}(3)-\mathrm{B}(8)$

135.4(3)

$\mathrm{B}(7)-\mathrm{B}(2)-\mathrm{B}(3)-\mathrm{B}(8)$

37.1(3)

$\mathrm{B}(6)-\mathrm{B}(2)-\mathrm{B}(3)-\mathrm{B}(8)$

100.4(3)

$\mathrm{C}(1)-\mathrm{B}(2)-\mathrm{B}(3)-\mathrm{B}(4)$

34.4(3)

$\mathrm{B}(7)-\mathrm{B}(2)-\mathrm{B}(3)-\mathrm{B}(4)$

$-63.9(3)$

$\mathrm{B}(8)-\mathrm{B}(2)-\mathrm{B}(3)-\mathrm{B}(4)$

$-101.0(3)$

$\mathrm{B}(6)-\mathrm{B}(2)-\mathrm{B}(3)-\mathrm{B}(4)$

$-0.5(4)$

$\mathrm{C}(1)-\mathrm{B}(2)-\mathrm{B}(3)-\mathrm{B}(9)$

97.8(3)

$\mathrm{B}(7)-\mathrm{B}(2)-\mathrm{B}(3)-\mathrm{B}(9)$

$-0.5(4)$

$\mathrm{B}(8)-\mathrm{B}(2)-\mathrm{B}(3)-\mathrm{B}(9)$

$-37.6(3)$

$\mathrm{B}(6)-\mathrm{B}(2)-\mathrm{B}(3)-\mathrm{B}(9)$

62.8(3)

$\mathrm{B}(5)-\mathrm{C}(1)-\mathrm{B}(4)-\mathrm{B}(9)$

101.8(3)

$\mathrm{B}(6)-\mathrm{C}(1)-\mathrm{B}(4)-\mathrm{B}(9)$

64.2(4)

$\mathrm{B}(2)-\mathrm{C}(1)-\mathrm{B}(4)-\mathrm{B}(9)$

$-2.9(4)$

$\mathrm{B}(3)-\mathrm{C}(1)-\mathrm{B}(4)-\mathrm{B}(9)$

$-39.2(3)$

$\mathrm{P}(1)-\mathrm{C}(1)-\mathrm{B}(4)-\mathrm{B}(9)$

$-144.8(3)$

$\mathrm{B}(5)-\mathrm{C}(1)-\mathrm{B}(4)-\mathrm{B}(3)$

141.1(3)

$\mathrm{B}(6)-\mathrm{C}(1)-\mathrm{B}(4)-\mathrm{B}(3)$

103.5(3)

$\mathrm{B}(2)-\mathrm{C}(1)-\mathrm{B}(4)-\mathrm{B}(3)$

36.3(3)

$\mathrm{P}(1)-\mathrm{C}(1)-\mathrm{B}(4)-\mathrm{B}(3)$

$-105.6(3)$

$\mathrm{B}(5)-\mathrm{C}(1)-\mathrm{B}(4)-\mathrm{B}(10)$

38.3(3)

$\mathrm{B}(6)-\mathrm{C}(1)-\mathrm{B}(4)-\mathrm{B}(10)$

$0.7(4)$

$\mathrm{B}(2)-\mathrm{C}(1)-\mathrm{B}(4)-\mathrm{B}(10)$

$-66.4(4)$

$\mathrm{B}(3)-\mathrm{C}(1)-\mathrm{B}(4)-\mathrm{B}(10)$

$-102.7(3)$

$\mathrm{P}(1)-\mathrm{C}(1)-\mathrm{B}(4)-\mathrm{B}(10)$

151.7(3)

$\mathrm{B}(6)-\mathrm{C}(1)-\mathrm{B}(4)-\mathrm{B}(5)$

$-37.6(3)$

$\mathrm{B}(2)-\mathrm{C}(1)-\mathrm{B}(4)-\mathrm{B}(5)$

$-104.8(3)$

$\mathrm{B}(3)-\mathrm{C}(1)-\mathrm{B}(4)-\mathrm{B}(5)$

$-141.1(3)$

$\mathrm{P}(1)-\mathrm{C}(1)-\mathrm{B}(4)-\mathrm{B}(5)$

113.3(3)

$\mathrm{B}(8)-\mathrm{B}(3)-\mathrm{B}(4)-\mathrm{C}(1)$

$-98.0(3)$

B(9)-B(3)-B(4)-C(1)

$-135.6(3)$

$\mathrm{B}(2)-\mathrm{B}(3)-\mathrm{B}(4)-\mathrm{C}(1)$

$-34.3(3)$

$\mathrm{C}(1)-\mathrm{B}(3)-\mathrm{B}(4)-\mathrm{B}(9)$

135.6(3)

$\mathrm{B}(8)-\mathrm{B}(3)-\mathrm{B}(4)-\mathrm{B}(9)$

37.6(3)

$\mathrm{B}(2)-\mathrm{B}(3)-\mathrm{B}(4)-\mathrm{B}(9)$

101.2(3)

$\mathrm{C}(1)-\mathrm{B}(3)-\mathrm{B}(4)-\mathrm{B}(10)$

97.8(3) 


\begin{tabular}{|c|c|}
\hline $\mathrm{B}(8)-\mathrm{B}(3)-\mathrm{B}(4)-\mathrm{B}(10)$ & $-0.1(4)$ \\
\hline $\mathrm{B}(9)-\mathrm{B}(3)-\mathrm{B}(4)-\mathrm{B}(10)$ & $-37.8(3)$ \\
\hline $\mathrm{B}(2)-\mathrm{B}(3)-\mathrm{B}(4)-\mathrm{B}(10)$ & $63.5(3)$ \\
\hline $\mathrm{C}(1)-\mathrm{B}(3)-\mathrm{B}(4)-\mathrm{B}(5)$ & $34.4(3)$ \\
\hline $\mathrm{B}(8)-\mathrm{B}(3)-\mathrm{B}(4)-\mathrm{B}(5)$ & $-63.5(4)$ \\
\hline $\mathrm{B}(9)-\mathrm{B}(3)-\mathrm{B}(4)-\mathrm{B}(5)$ & $-101.1(3)$ \\
\hline $\mathrm{B}(2)-\mathrm{B}(3)-\mathrm{B}(4)-\mathrm{B}(5)$ & $0.1(4)$ \\
\hline $\mathrm{B}(6)-\mathrm{C}(1)-\mathrm{B}(5)-\mathrm{B}(10)$ & 101.9(3) \\
\hline $\mathrm{B}(2)-\mathrm{C}(1)-\mathrm{B}(5)-\mathrm{B}(10)$ & $65.2(4)$ \\
\hline $\mathrm{B}(4)-\mathrm{C}(1)-\mathrm{B}(5)-\mathrm{B}(10)$ & $-38.4(3)$ \\
\hline $\mathrm{B}(3)-\mathrm{C}(1)-\mathrm{B}(5)-\mathrm{B}(10)$ & $-1.9(4)$ \\
\hline $\mathrm{P}(1)-\mathrm{C}(1)-\mathrm{B}(5)-\mathrm{B}(10)$ & $-145.5(3)$ \\
\hline $\mathrm{B}(6)-\mathrm{C}(1)-\mathrm{B}(5)-\mathrm{B}(11)$ & $38.7(3)$ \\
\hline $\mathrm{B}(2)-\mathrm{C}(1)-\mathrm{B}(5)-\mathrm{B}(11)$ & $2.0(4)$ \\
\hline $\mathrm{B}(4)-\mathrm{C}(1)-\mathrm{B}(5)-\mathrm{B}(11)$ & $-101.5(3)$ \\
\hline $\mathrm{B}(3)-\mathrm{C}(1)-\mathrm{B}(5)-\mathrm{B}(11)$ & $-65.0(4)$ \\
\hline $\mathrm{P}(1)-\mathrm{C}(1)-\mathrm{B}(5)-\mathrm{B}(11)$ & $151.4(3)$ \\
\hline $\mathrm{B}(2)-\mathrm{C}(1)-\mathrm{B}(5)-\mathrm{B}(6)$ & $-36.7(3)$ \\
\hline $\mathrm{B}(4)-\mathrm{C}(1)-\mathrm{B}(5)-\mathrm{B}(6)$ & $-140.2(3)$ \\
\hline $\mathrm{B}(3)-\mathrm{C}(1)-\mathrm{B}(5)-\mathrm{B}(6)$ & $-103.8(4)$ \\
\hline $\mathrm{P}(1)-\mathrm{C}(1)-\mathrm{B}(5)-\mathrm{B}(6)$ & $112.7(4)$ \\
\hline $\mathrm{B}(6)-\mathrm{C}(1)-\mathrm{B}(5)-\mathrm{B}(4)$ & $140.2(3)$ \\
\hline $\mathrm{B}(2)-\mathrm{C}(1)-\mathrm{B}(5)-\mathrm{B}(4)$ & $103.5(3)$ \\
\hline $\mathrm{B}(3)-\mathrm{C}(1)-\mathrm{B}(5)-\mathrm{B}(4)$ & $36.5(3)$ \\
\hline $\mathrm{P}(1)-\mathrm{C}(1)-\mathrm{B}(5)-\mathrm{B}(4)$ & $-107.1(4)$ \\
\hline $\mathrm{B}(9)-\mathrm{B}(4)-\mathrm{B}(5)-\mathrm{C}(1)$ & $-98.5(3)$ \\
\hline $\mathrm{B}(3)-\mathrm{B}(4)-\mathrm{B}(5)-\mathrm{C}(1)$ & $-34.8(3)$ \\
\hline $\mathrm{B}(10)-\mathrm{B}(4)-\mathrm{B}(5)-\mathrm{C}(1)$ & $-136.3(3)$ \\
\hline $\mathrm{C}(1)-\mathrm{B}(4)-\mathrm{B}(5)-\mathrm{B}(10)$ & $136.3(3)$ \\
\hline $\mathrm{B}(9)-\mathrm{B}(4)-\mathrm{B}(5)-\mathrm{B}(10)$ & $37.9(3)$ \\
\hline $\mathrm{B}(3)-\mathrm{B}(4)-\mathrm{B}(5)-\mathrm{B}(10)$ & $101.5(3)$ \\
\hline $\mathrm{C}(1)-\mathrm{B}(4)-\mathrm{B}(5)-\mathrm{B}(11)$ & $98.2(3)$ \\
\hline $\mathrm{B}(9)-\mathrm{B}(4)-\mathrm{B}(5)-\mathrm{B}(11)$ & $-0.3(4)$ \\
\hline $\mathrm{B}(3)-\mathrm{B}(4)-\mathrm{B}(5)-\mathrm{B}(11)$ & $63.4(4)$ \\
\hline $\mathrm{B}(10)-\mathrm{B}(4)-\mathrm{B}(5)-\mathrm{B}(11)$ & $-38.2(3)$ \\
\hline $\mathrm{C}(1)-\mathrm{B}(4)-\mathrm{B}(5)-\mathrm{B}(6)$ & $35.2(3)$ \\
\hline
\end{tabular}




\begin{tabular}{|c|c|}
\hline $\mathrm{B}(9)-\mathrm{B}(4)-\mathrm{B}(5)-\mathrm{B}(6)$ & $-63.3(4)$ \\
\hline $\mathrm{B}(3)-\mathrm{B}(4)-\mathrm{B}(5)-\mathrm{B}(6)$ & $0.4(4)$ \\
\hline $\mathrm{B}(10)-\mathrm{B}(4)-\mathrm{B}(5)-\mathrm{B}(6)$ & $-101.1(3)$ \\
\hline $\mathrm{B}(5)-\mathrm{C}(1)-\mathrm{B}(6)-\mathrm{B}(11)$ & $-38.9(3)$ \\
\hline $\mathrm{B}(2)-\mathrm{C}(1)-\mathrm{B}(6)-\mathrm{B}(11)$ & $102.3(3)$ \\
\hline $\mathrm{B}(4)-\mathrm{C}(1)-\mathrm{B}(6)-\mathrm{B}(11)$ & $-1.2(4)$ \\
\hline $\mathrm{B}(3)-\mathrm{C}(1)-\mathrm{B}(6)-\mathrm{B}(11)$ & $65.4(4)$ \\
\hline $\mathrm{P}(1)-\mathrm{C}(1)-\mathrm{B}(6)-\mathrm{B}(11)$ & $-150.6(3)$ \\
\hline $\mathrm{B}(5)-\mathrm{C}(1)-\mathrm{B}(6)-\mathrm{B}(7)$ & $-102.1(3)$ \\
\hline $\mathrm{B}(2)-\mathrm{C}(1)-\mathrm{B}(6)-\mathrm{B}(7)$ & $39.0(3)$ \\
\hline $\mathrm{B}(4)-\mathrm{C}(1)-\mathrm{B}(6)-\mathrm{B}(7)$ & $-64.4(4)$ \\
\hline $\mathrm{B}(3)-\mathrm{C}(1)-\mathrm{B}(6)-\mathrm{B}(7)$ & $2.1(4)$ \\
\hline $\mathrm{P}(1)-\mathrm{C}(1)-\mathrm{B}(6)-\mathrm{B}(7)$ & $146.2(3)$ \\
\hline $\mathrm{B}(5)-\mathrm{C}(1)-\mathrm{B}(6)-\mathrm{B}(2)$ & $-141.2(3)$ \\
\hline $\mathrm{B}(4)-\mathrm{C}(1)-\mathrm{B}(6)-\mathrm{B}(2)$ & $-103.5(3)$ \\
\hline $\mathrm{B}(3)-\mathrm{C}(1)-\mathrm{B}(6)-\mathrm{B}(2)$ & $-36.9(3)$ \\
\hline $\mathrm{P}(1)-\mathrm{C}(1)-\mathrm{B}(6)-\mathrm{B}(2)$ & $107.2(3)$ \\
\hline $\mathrm{B}(2)-\mathrm{C}(1)-\mathrm{B}(6)-\mathrm{B}(5)$ & $141.2(3)$ \\
\hline $\mathrm{B}(4)-\mathrm{C}(1)-\mathrm{B}(6)-\mathrm{B}(5)$ & $37.7(3)$ \\
\hline $\mathrm{B}(3)-\mathrm{C}(1)-\mathrm{B}(6)-\mathrm{B}(5)$ & $104.3(3)$ \\
\hline $\mathrm{P}(1)-\mathrm{C}(1)-\mathrm{B}(6)-\mathrm{B}(5)$ & $-111.7(4)$ \\
\hline $\mathrm{B}(7)-\mathrm{B}(2)-\mathrm{B}(6)-\mathrm{C}(1)$ & $135.7(3)$ \\
\hline $\mathrm{B}(3)-\mathrm{B}(2)-\mathrm{B}(6)-\mathrm{C}(1)$ & $35.1(3)$ \\
\hline $\mathrm{B}(8)-\mathrm{B}(2)-\mathrm{B}(6)-\mathrm{C}(1)$ & $98.2(3)$ \\
\hline $\mathrm{C}(1)-\mathrm{B}(2)-\mathrm{B}(6)-\mathrm{B}(11)$ & $-98.0(3)$ \\
\hline $\mathrm{B}(7)-\mathrm{B}(2)-\mathrm{B}(6)-\mathrm{B}(11)$ & $37.7(3)$ \\
\hline $\mathrm{B}(3)-\mathrm{B}(2)-\mathrm{B}(6)-\mathrm{B}(11)$ & $-62.8(4)$ \\
\hline $\mathrm{B}(8)-\mathrm{B}(2)-\mathrm{B}(6)-\mathrm{B}(11)$ & $0.3(4)$ \\
\hline $\mathrm{C}(1)-\mathrm{B}(2)-\mathrm{B}(6)-\mathrm{B}(7)$ & $-135.7(3)$ \\
\hline $\mathrm{B}(3)-\mathrm{B}(2)-\mathrm{B}(6)-\mathrm{B}(7)$ & $-100.5(3)$ \\
\hline $\mathrm{B}(8)-\mathrm{B}(2)-\mathrm{B}(6)-\mathrm{B}(7)$ & $-37.5(3)$ \\
\hline $\mathrm{C}(1)-\mathrm{B}(2)-\mathrm{B}(6)-\mathrm{B}(5)$ & $-34.4(3)$ \\
\hline $\mathrm{B}(7)-\mathrm{B}(2)-\mathrm{B}(6)-\mathrm{B}(5)$ & 101.3(3) \\
\hline $\mathrm{B}(3)-\mathrm{B}(2)-\mathrm{B}(6)-\mathrm{B}(5)$ & $0.8(4)$ \\
\hline $\mathrm{B}(8)-\mathrm{B}(2)-\mathrm{B}(6)-\mathrm{B}(5)$ & $63.8(4)$ \\
\hline $\mathrm{B}(10)-\mathrm{B}(5)-\mathrm{B}(6)-\mathrm{C}(1)$ & $-98.3(3)$ \\
\hline
\end{tabular}




\begin{tabular}{|c|c|}
\hline $\mathrm{B}(11)-\mathrm{B}(5)-\mathrm{B}(6)-\mathrm{C}(1)$ & $-135.8(3)$ \\
\hline $\mathrm{B}(4)-\mathrm{B}(5)-\mathrm{B}(6)-\mathrm{C}(1)$ & $-35.2(3)$ \\
\hline $\mathrm{C}(1)-\mathrm{B}(5)-\mathrm{B}(6)-\mathrm{B}(11)$ & $135.8(3)$ \\
\hline $\mathrm{B}(10)-\mathrm{B}(5)-\mathrm{B}(6)-\mathrm{B}(11)$ & $37.5(3)$ \\
\hline $\mathrm{B}(4)-\mathrm{B}(5)-\mathrm{B}(6)-\mathrm{B}(11)$ & $100.6(3)$ \\
\hline $\mathrm{C}(1)-\mathrm{B}(5)-\mathrm{B}(6)-\mathrm{B}(7)$ & $98.0(3)$ \\
\hline $\mathrm{B}(10)-\mathrm{B}(5)-\mathrm{B}(6)-\mathrm{B}(7)$ & $-0.3(4)$ \\
\hline $\mathrm{B}(11)-\mathrm{B}(5)-\mathrm{B}(6)-\mathrm{B}(7)$ & $-37.8(3)$ \\
\hline $\mathrm{B}(4)-\mathrm{B}(5)-\mathrm{B}(6)-\mathrm{B}(7)$ & $62.8(4)$ \\
\hline $\mathrm{C}(1)-\mathrm{B}(5)-\mathrm{B}(6)-\mathrm{B}(2)$ & $34.5(3)$ \\
\hline $\mathrm{B}(10)-\mathrm{B}(5)-\mathrm{B}(6)-\mathrm{B}(2)$ & $-63.8(4)$ \\
\hline $\mathrm{B}(11)-\mathrm{B}(5)-\mathrm{B}(6)-\mathrm{B}(2)$ & $-101.3(3)$ \\
\hline $\mathrm{B}(4)-\mathrm{B}(5)-\mathrm{B}(6)-\mathrm{B}(2)$ & $-0.7(4)$ \\
\hline $\mathrm{C}(1)-\mathrm{B}(6)-\mathrm{B}(7)-\mathrm{B}(8)$ & $-1.1(4)$ \\
\hline $\mathrm{B}(11)-\mathrm{B}(6)-\mathrm{B}(7)-\mathrm{B}(8)$ & $-100.7(4)$ \\
\hline $\mathrm{B}(2)-\mathrm{B}(6)-\mathrm{B}(7)-\mathrm{B}(8)$ & $37.5(3)$ \\
\hline $\mathrm{B}(5)-\mathrm{B}(6)-\mathrm{B}(7)-\mathrm{B}(8)$ & $-63.0(4)$ \\
\hline $\mathrm{C}(1)-\mathrm{B}(6)-\mathrm{B}(7)-\mathrm{B}(2)$ & $-38.6(3)$ \\
\hline $\mathrm{B}(11)-\mathrm{B}(6)-\mathrm{B}(7)-\mathrm{B}(2)$ & $-138.2(3)$ \\
\hline $\mathrm{B}(5)-\mathrm{B}(6)-\mathrm{B}(7)-\mathrm{B}(2)$ & $-100.6(3)$ \\
\hline $\mathrm{C}(1)-\mathrm{B}(6)-\mathrm{B}(7)-\mathrm{B}(12)$ & $62.1(4)$ \\
\hline $\mathrm{B}(11)-\mathrm{B}(6)-\mathrm{B}(7)-\mathrm{B}(12)$ & $-37.5(3)$ \\
\hline $\mathrm{B}(2)-\mathrm{B}(6)-\mathrm{B}(7)-\mathrm{B}(12)$ & $100.7(3)$ \\
\hline $\mathrm{B}(5)-\mathrm{B}(6)-\mathrm{B}(7)-\mathrm{B}(12)$ & $0.2(4)$ \\
\hline $\mathrm{C}(1)-\mathrm{B}(6)-\mathrm{B}(7)-\mathrm{B}(11)$ & $99.5(3)$ \\
\hline $\mathrm{B}(2)-\mathrm{B}(6)-\mathrm{B}(7)-\mathrm{B}(11)$ & $138.2(3)$ \\
\hline $\mathrm{B}(5)-\mathrm{B}(6)-\mathrm{B}(7)-\mathrm{B}(11)$ & $37.6(3)$ \\
\hline $\mathrm{C}(1)-\mathrm{B}(2)-\mathrm{B}(7)-\mathrm{B}(6)$ & $38.6(3)$ \\
\hline $\mathrm{B}(3)-\mathrm{B}(2)-\mathrm{B}(7)-\mathrm{B}(6)$ & $101.1(3)$ \\
\hline $\mathrm{B}(8)-\mathrm{B}(2)-\mathrm{B}(7)-\mathrm{B}(6)$ & $138.0(3)$ \\
\hline $\mathrm{C}(1)-\mathrm{B}(2)-\mathrm{B}(7)-\mathrm{B}(8)$ & $-99.5(3)$ \\
\hline $\mathrm{B}(3)-\mathrm{B}(2)-\mathrm{B}(7)-\mathrm{B}(8)$ & $-37.0(3)$ \\
\hline $\mathrm{B}(6)-\mathrm{B}(2)-\mathrm{B}(7)-\mathrm{B}(8)$ & $-138.0(3)$ \\
\hline $\mathrm{C}(1)-\mathrm{B}(2)-\mathrm{B}(7)-\mathrm{B}(12)$ & $-61.9(4)$ \\
\hline $\mathrm{B}(3)-\mathrm{B}(2)-\mathrm{B}(7)-\mathrm{B}(12)$ & $0.6(4)$ \\
\hline $\mathrm{B}(8)-\mathrm{B}(2)-\mathrm{B}(7)-\mathrm{B}(12)$ & $37.6(3)$ \\
\hline
\end{tabular}




\begin{tabular}{|c|c|}
\hline $\mathrm{B}(6)-\mathrm{B}(2)-\mathrm{B}(7)-\mathrm{B}(12)$ & $-100.5(3)$ \\
\hline $\mathrm{C}(1)-\mathrm{B}(2)-\mathrm{B}(7)-\mathrm{B}(11)$ & $1.4(4)$ \\
\hline $\mathrm{B}(3)-\mathrm{B}(2)-\mathrm{B}(7)-\mathrm{B}(11)$ & $63.9(4)$ \\
\hline $\mathrm{B}(8)-\mathrm{B}(2)-\mathrm{B}(7)-\mathrm{B}(11)$ & $100.9(4)$ \\
\hline $\mathrm{B}(6)-\mathrm{B}(2)-\mathrm{B}(7)-\mathrm{B}(11)$ & $-37.1(3)$ \\
\hline $\mathrm{C}(1)-\mathrm{B}(3)-\mathrm{B}(8)-\mathrm{B}(7)$ & $1.5(4)$ \\
\hline $\mathrm{B}(4)-\mathrm{B}(3)-\mathrm{B}(8)-\mathrm{B}(7)$ & $63.6(4)$ \\
\hline $\mathrm{B}(9)-\mathrm{B}(3)-\mathrm{B}(8)-\mathrm{B}(7)$ & $101.0(3)$ \\
\hline $\mathrm{B}(2)-\mathrm{B}(3)-\mathrm{B}(8)-\mathrm{B}(7)$ & $-37.2(3)$ \\
\hline $\mathrm{C}(1)-\mathrm{B}(3)-\mathrm{B}(8)-\mathrm{B}(12)$ & $-62.2(4)$ \\
\hline $\mathrm{B}(4)-\mathrm{B}(3)-\mathrm{B}(8)-\mathrm{B}(12)$ & $-0.2(4)$ \\
\hline $\mathrm{B}(9)-\mathrm{B}(3)-\mathrm{B}(8)-\mathrm{B}(12)$ & $37.2(3)$ \\
\hline $\mathrm{B}(2)-\mathrm{B}(3)-\mathrm{B}(8)-\mathrm{B}(12)$ & $-101.0(3)$ \\
\hline $\mathrm{C}(1)-\mathrm{B}(3)-\mathrm{B}(8)-\mathrm{B}(2)$ & $38.7(3)$ \\
\hline $\mathrm{B}(4)-\mathrm{B}(3)-\mathrm{B}(8)-\mathrm{B}(2)$ & $100.8(3)$ \\
\hline $\mathrm{B}(9)-\mathrm{B}(3)-\mathrm{B}(8)-\mathrm{B}(2)$ & $138.2(3)$ \\
\hline $\mathrm{C}(1)-\mathrm{B}(3)-\mathrm{B}(8)-\mathrm{B}(9)$ & $-99.5(3)$ \\
\hline $\mathrm{B}(4)-\mathrm{B}(3)-\mathrm{B}(8)-\mathrm{B}(9)$ & $-37.4(3)$ \\
\hline $\mathrm{B}(2)-\mathrm{B}(3)-\mathrm{B}(8)-\mathrm{B}(9)$ & $-138.2(3)$ \\
\hline $\mathrm{B}(6)-\mathrm{B}(7)-\mathrm{B}(8)-\mathrm{B}(3)$ & $-0.3(4)$ \\
\hline $\mathrm{B}(2)-\mathrm{B}(7)-\mathrm{B}(8)-\mathrm{B}(3)$ & $37.3(3)$ \\
\hline $\mathrm{B}(12)-\mathrm{B}(7)-\mathrm{B}(8)-\mathrm{B}(3)$ & $-100.5(3)$ \\
\hline $\mathrm{B}(11)-\mathrm{B}(7)-\mathrm{B}(8)-\mathrm{B}(3)$ & $-63.2(4)$ \\
\hline $\mathrm{B}(6)-\mathrm{B}(7)-\mathrm{B}(8)-\mathrm{B}(12)$ & $100.3(3)$ \\
\hline $\mathrm{B}(2)-\mathrm{B}(7)-\mathrm{B}(8)-\mathrm{B}(12)$ & $137.8(3)$ \\
\hline $\mathrm{B}(11)-\mathrm{B}(7)-\mathrm{B}(8)-\mathrm{B}(12)$ & $37.3(3)$ \\
\hline $\mathrm{B}(6)-\mathrm{B}(7)-\mathrm{B}(8)-\mathrm{B}(2)$ & $-37.5(3)$ \\
\hline $\mathrm{B}(12)-\mathrm{B}(7)-\mathrm{B}(8)-\mathrm{B}(2)$ & $-137.8(3)$ \\
\hline $\mathrm{B}(11)-\mathrm{B}(7)-\mathrm{B}(8)-\mathrm{B}(2)$ & $-100.5(3)$ \\
\hline $\mathrm{B}(6)-\mathrm{B}(7)-\mathrm{B}(8)-\mathrm{B}(9)$ & $63.2(4)$ \\
\hline $\mathrm{B}(2)-\mathrm{B}(7)-\mathrm{B}(8)-\mathrm{B}(9)$ & $100.7(3)$ \\
\hline $\mathrm{B}(12)-\mathrm{B}(7)-\mathrm{B}(8)-\mathrm{B}(9)$ & $-37.1(3)$ \\
\hline $\mathrm{B}(11)-\mathrm{B}(7)-\mathrm{B}(8)-\mathrm{B}(9)$ & $0.2(4)$ \\
\hline $\mathrm{C}(1)-\mathrm{B}(2)-\mathrm{B}(8)-\mathrm{B}(3)$ & $-39.0(3)$ \\
\hline $\mathrm{B}(7)-\mathrm{B}(2)-\mathrm{B}(8)-\mathrm{B}(3)$ & $-138.5(3)$ \\
\hline $\mathrm{B}(6)-\mathrm{B}(2)-\mathrm{B}(8)-\mathrm{B}(3)$ & $-101.0(3)$ \\
\hline
\end{tabular}




\begin{tabular}{|c|c|}
\hline $\mathrm{C}(1)-\mathrm{B}(2)-\mathrm{B}(8)-\mathrm{B}(7)$ & $99.4(3)$ \\
\hline $\mathrm{B}(3)-\mathrm{B}(2)-\mathrm{B}(8)-\mathrm{B}(7)$ & $138.5(3)$ \\
\hline $\mathrm{B}(6)-\mathrm{B}(2)-\mathrm{B}(8)-\mathrm{B}(7)$ & $37.4(3)$ \\
\hline $\mathrm{C}(1)-\mathrm{B}(2)-\mathrm{B}(8)-\mathrm{B}(12)$ & $61.6(4)$ \\
\hline $\mathrm{B}(7)-\mathrm{B}(2)-\mathrm{B}(8)-\mathrm{B}(12)$ & $-37.8(3)$ \\
\hline $\mathrm{B}(3)-\mathrm{B}(2)-\mathrm{B}(8)-\mathrm{B}(12)$ & $100.6(3)$ \\
\hline $\mathrm{B}(6)-\mathrm{B}(2)-\mathrm{B}(8)-\mathrm{B}(12)$ & $-0.4(4)$ \\
\hline $\mathrm{C}(1)-\mathrm{B}(2)-\mathrm{B}(8)-\mathrm{B}(9)$ & $-1.7(4)$ \\
\hline $\mathrm{B}(7)-\mathrm{B}(2)-\mathrm{B}(8)-\mathrm{B}(9)$ & $-101.2(3)$ \\
\hline $\mathrm{B}(3)-\mathrm{B}(2)-\mathrm{B}(8)-\mathrm{B}(9)$ & $37.3(3)$ \\
\hline $\mathrm{B}(6)-\mathrm{B}(2)-\mathrm{B}(8)-\mathrm{B}(9)$ & $-63.7(4)$ \\
\hline $\mathrm{C}(1)-\mathrm{B}(4)-\mathrm{B}(9)-\mathrm{B}(3)$ & $39.0(3)$ \\
\hline $\mathrm{B}(10)-\mathrm{B}(4)-\mathrm{B}(9)-\mathrm{B}(3)$ & $138.3(3)$ \\
\hline $\mathrm{B}(5)-\mathrm{B}(4)-\mathrm{B}(9)-\mathrm{B}(3)$ & $100.9(3)$ \\
\hline $\mathrm{C}(1)-\mathrm{B}(4)-\mathrm{B}(9)-\mathrm{B}(12)$ & $-61.7(4)$ \\
\hline $\mathrm{B}(3)-\mathrm{B}(4)-\mathrm{B}(9)-\mathrm{B}(12)$ & $-100.7(3)$ \\
\hline $\mathrm{B}(10)-\mathrm{B}(4)-\mathrm{B}(9)-\mathrm{B}(12)$ & $37.6(3)$ \\
\hline $\mathrm{B}(5)-\mathrm{B}(4)-\mathrm{B}(9)-\mathrm{B}(12)$ & $0.2(4)$ \\
\hline $\mathrm{C}(1)-\mathrm{B}(4)-\mathrm{B}(9)-\mathrm{B}(8)$ & $1.7(4)$ \\
\hline $\mathrm{B}(3)-\mathrm{B}(4)-\mathrm{B}(9)-\mathrm{B}(8)$ & $-37.3(3)$ \\
\hline $\mathrm{B}(10)-\mathrm{B}(4)-\mathrm{B}(9)-\mathrm{B}(8)$ & $101.0(3)$ \\
\hline $\mathrm{B}(5)-\mathrm{B}(4)-\mathrm{B}(9)-\mathrm{B}(8)$ & $63.6(4)$ \\
\hline $\mathrm{C}(1)-\mathrm{B}(4)-\mathrm{B}(9)-\mathrm{B}(10)$ & $-99.3(3)$ \\
\hline $\mathrm{B}(3)-\mathrm{B}(4)-\mathrm{B}(9)-\mathrm{B}(10)$ & $-138.3(3)$ \\
\hline $\mathrm{B}(5)-\mathrm{B}(4)-\mathrm{B}(9)-\mathrm{B}(10)$ & $-37.5(3)$ \\
\hline $\mathrm{C}(1)-\mathrm{B}(3)-\mathrm{B}(9)-\mathrm{B}(4)$ & $-38.6(3)$ \\
\hline $\mathrm{B}(8)-\mathrm{B}(3)-\mathrm{B}(9)-\mathrm{B}(4)$ & $-138.1(3)$ \\
\hline $\mathrm{B}(2)-\mathrm{B}(3)-\mathrm{B}(9)-\mathrm{B}(4)$ & $-100.6(3)$ \\
\hline $\mathrm{C}(1)-\mathrm{B}(3)-\mathrm{B}(9)-\mathrm{B}(12)$ & $62.2(4)$ \\
\hline $\mathrm{B}(8)-\mathrm{B}(3)-\mathrm{B}(9)-\mathrm{B}(12)$ & $-37.3(3)$ \\
\hline $\mathrm{B}(4)-\mathrm{B}(3)-\mathrm{B}(9)-\mathrm{B}(12)$ & $100.8(3)$ \\
\hline $\mathrm{B}(2)-\mathrm{B}(3)-\mathrm{B}(9)-\mathrm{B}(12)$ & $0.2(4)$ \\
\hline $\mathrm{C}(1)-\mathrm{B}(3)-\mathrm{B}(9)-\mathrm{B}(8)$ & $99.5(3)$ \\
\hline $\mathrm{B}(4)-\mathrm{B}(3)-\mathrm{B}(9)-\mathrm{B}(8)$ & 138.1(3) \\
\hline $\mathrm{B}(2)-\mathrm{B}(3)-\mathrm{B}(9)-\mathrm{B}(8)$ & $37.5(3)$ \\
\hline $\mathrm{C}(1)-\mathrm{B}(3)-\mathrm{B}(9)-\mathrm{B}(10)$ & $-1.5(4)$ \\
\hline
\end{tabular}




\begin{tabular}{|c|c|}
\hline $\mathrm{B}(8)-\mathrm{B}(3)-\mathrm{B}(9)-\mathrm{B}(10)$ & $-100.9(3)$ \\
\hline $\mathrm{B}(4)-\mathrm{B}(3)-\mathrm{B}(9)-\mathrm{B}(10)$ & $37.2(3)$ \\
\hline $\mathrm{B}(2)-\mathrm{B}(3)-\mathrm{B}(9)-\mathrm{B}(10)$ & $-63.4(4)$ \\
\hline $\mathrm{B}(3)-\mathrm{B}(8)-\mathrm{B}(9)-\mathrm{B}(4)$ & $37.4(3)$ \\
\hline $\mathrm{B}(7)-\mathrm{B}(8)-\mathrm{B}(9)-\mathrm{B}(4)$ & $-63.5(4)$ \\
\hline $\mathrm{B}(12)-\mathrm{B}(8)-\mathrm{B}(9)-\mathrm{B}(4)$ & $-100.8(3)$ \\
\hline $\mathrm{B}(2)-\mathrm{B}(8)-\mathrm{B}(9)-\mathrm{B}(4)$ & $0.0(4)$ \\
\hline $\mathrm{B}(7)-\mathrm{B}(8)-\mathrm{B}(9)-\mathrm{B}(3)$ & $-100.9(3)$ \\
\hline $\mathrm{B}(12)-\mathrm{B}(8)-\mathrm{B}(9)-\mathrm{B}(3)$ & $-138.2(3)$ \\
\hline $\mathrm{B}(2)-\mathrm{B}(8)-\mathrm{B}(9)-\mathrm{B}(3)$ & $-37.4(3)$ \\
\hline $\mathrm{B}(3)-\mathrm{B}(8)-\mathrm{B}(9)-\mathrm{B}(12)$ & $138.2(3)$ \\
\hline $\mathrm{B}(7)-\mathrm{B}(8)-\mathrm{B}(9)-\mathrm{B}(12)$ & $37.3(3)$ \\
\hline $\mathrm{B}(2)-\mathrm{B}(8)-\mathrm{B}(9)-\mathrm{B}(12)$ & $100.8(3)$ \\
\hline $\mathrm{B}(3)-\mathrm{B}(8)-\mathrm{B}(9)-\mathrm{B}(10)$ & $100.7(3)$ \\
\hline $\mathrm{B}(7)-\mathrm{B}(8)-\mathrm{B}(9)-\mathrm{B}(10)$ & $-0.3(4)$ \\
\hline $\mathrm{B}(12)-\mathrm{B}(8)-\mathrm{B}(9)-\mathrm{B}(10)$ & $-37.5(3)$ \\
\hline $\mathrm{B}(2)-\mathrm{B}(8)-\mathrm{B}(9)-\mathrm{B}(10)$ & $63.3(4)$ \\
\hline $\mathrm{C}(1)-\mathrm{B}(4)-\mathrm{B}(10)-\mathrm{B}(5)$ & $-37.8(3)$ \\
\hline $\mathrm{B}(9)-\mathrm{B}(4)-\mathrm{B}(10)-\mathrm{B}(5)$ & $-138.1(3)$ \\
\hline $\mathrm{B}(3)-\mathrm{B}(4)-\mathrm{B}(10)-\mathrm{B}(5)$ & $-100.6(3)$ \\
\hline $\mathrm{C}(1)-\mathrm{B}(4)-\mathrm{B}(10)-\mathrm{B}(12)$ & $63.2(4)$ \\
\hline $\mathrm{B}(9)-\mathrm{B}(4)-\mathrm{B}(10)-\mathrm{B}(12)$ & $-37.1(3)$ \\
\hline $\mathrm{B}(3)-\mathrm{B}(4)-\mathrm{B}(10)-\mathrm{B}(12)$ & $0.4(4)$ \\
\hline $\mathrm{B}(5)-\mathrm{B}(4)-\mathrm{B}(10)-\mathrm{B}(12)$ & $101.0(4)$ \\
\hline $\mathrm{C}(1)-\mathrm{B}(4)-\mathrm{B}(10)-\mathrm{B}(11)$ & $0.1(4)$ \\
\hline $\mathrm{B}(9)-\mathrm{B}(4)-\mathrm{B}(10)-\mathrm{B}(11)$ & $-100.2(3)$ \\
\hline $\mathrm{B}(3)-\mathrm{B}(4)-\mathrm{B}(10)-\mathrm{B}(11)$ & $-62.7(3)$ \\
\hline $\mathrm{B}(5)-\mathrm{B}(4)-\mathrm{B}(10)-\mathrm{B}(11)$ & $37.9(3)$ \\
\hline $\mathrm{C}(1)-\mathrm{B}(4)-\mathrm{B}(10)-\mathrm{B}(9)$ & $100.2(3)$ \\
\hline $\mathrm{B}(3)-\mathrm{B}(4)-\mathrm{B}(10)-\mathrm{B}(9)$ & $37.5(3)$ \\
\hline $\mathrm{B}(5)-\mathrm{B}(4)-\mathrm{B}(10)-\mathrm{B}(9)$ & $138.1(3)$ \\
\hline $\mathrm{C}(1)-\mathrm{B}(5)-\mathrm{B}(10)-\mathrm{B}(4)$ & $38.1(3)$ \\
\hline $\mathrm{B}(11)-\mathrm{B}(5)-\mathrm{B}(10)-\mathrm{B}(4)$ & $137.4(3)$ \\
\hline $\mathrm{B}(6)-\mathrm{B}(5)-\mathrm{B}(10)-\mathrm{B}(4)$ & $100.3(3)$ \\
\hline $\mathrm{C}(1)-\mathrm{B}(5)-\mathrm{B}(10)-\mathrm{B}(12)$ & $-61.9(4)$ \\
\hline $\mathrm{B}(11)-\mathrm{B}(5)-\mathrm{B}(10)-\mathrm{B}(12)$ & $37.5(3)$ \\
\hline
\end{tabular}




\begin{tabular}{|c|c|}
\hline $\mathrm{B}(6)-\mathrm{B}(5)-\mathrm{B}(10)-\mathrm{B}(12)$ & $0.3(4)$ \\
\hline $\mathrm{B}(4)-\mathrm{B}(5)-\mathrm{B}(10)-\mathrm{B}(12)$ & $-99.9(3)$ \\
\hline $\mathrm{C}(1)-\mathrm{B}(5)-\mathrm{B}(10)-\mathrm{B}(11)$ & $-99.4(3)$ \\
\hline $\mathrm{B}(6)-\mathrm{B}(5)-\mathrm{B}(10)-\mathrm{B}(11)$ & $-37.2(3)$ \\
\hline $\mathrm{B}(4)-\mathrm{B}(5)-\mathrm{B}(10)-\mathrm{B}(11)$ & $-137.4(3)$ \\
\hline $\mathrm{C}(1)-\mathrm{B}(5)-\mathrm{B}(10)-\mathrm{B}(9)$ & $0.9(4)$ \\
\hline $\mathrm{B}(11)-\mathrm{B}(5)-\mathrm{B}(10)-\mathrm{B}(9)$ & $100.3(3)$ \\
\hline $\mathrm{B}(6)-\mathrm{B}(5)-\mathrm{B}(10)-\mathrm{B}(9)$ & $63.1(4)$ \\
\hline $\mathrm{B}(4)-\mathrm{B}(5)-\mathrm{B}(10)-\mathrm{B}(9)$ & $-37.2(3)$ \\
\hline $\mathrm{B}(3)-\mathrm{B}(9)-\mathrm{B}(10)-\mathrm{B}(4)$ & $-37.3(3)$ \\
\hline $\mathrm{B}(12)-\mathrm{B}(9)-\mathrm{B}(10)-\mathrm{B}(4)$ & $-138.0(3)$ \\
\hline $\mathrm{B}(8)-\mathrm{B}(9)-\mathrm{B}(10)-\mathrm{B}(4)$ & $-100.5(3)$ \\
\hline $\mathrm{B}(4)-\mathrm{B}(9)-\mathrm{B}(10)-\mathrm{B}(5)$ & $37.6(3)$ \\
\hline $\mathrm{B}(3)-\mathrm{B}(9)-\mathrm{B}(10)-\mathrm{B}(5)$ & $0.4(4)$ \\
\hline $\mathrm{B}(12)-\mathrm{B}(9)-\mathrm{B}(10)-\mathrm{B}(5)$ & $-100.4(4)$ \\
\hline $\mathrm{B}(8)-\mathrm{B}(9)-\mathrm{B}(10)-\mathrm{B}(5)$ & $-62.9(4)$ \\
\hline $\mathrm{B}(4)-\mathrm{B}(9)-\mathrm{B}(10)-\mathrm{B}(12)$ & $138.0(3)$ \\
\hline $\mathrm{B}(3)-\mathrm{B}(9)-\mathrm{B}(10)-\mathrm{B}(12)$ & $100.7(4)$ \\
\hline $\mathrm{B}(8)-\mathrm{B}(9)-\mathrm{B}(10)-\mathrm{B}(12)$ & $37.5(3)$ \\
\hline $\mathrm{B}(4)-\mathrm{B}(9)-\mathrm{B}(10)-\mathrm{B}(11)$ & $100.8(3)$ \\
\hline $\mathrm{B}(3)-\mathrm{B}(9)-\mathrm{B}(10)-\mathrm{B}(11)$ & $63.5(4)$ \\
\hline $\mathrm{B}(12)-\mathrm{B}(9)-\mathrm{B}(10)-\mathrm{B}(11)$ & $-37.2(3)$ \\
\hline $\mathrm{B}(8)-\mathrm{B}(9)-\mathrm{B}(10)-\mathrm{B}(11)$ & $0.2(4)$ \\
\hline $\mathrm{C}(1)-\mathrm{B}(6)-\mathrm{B}(11)-\mathrm{B}(5)$ & $38.4(3)$ \\
\hline $\mathrm{B}(7)-\mathrm{B}(6)-\mathrm{B}(11)-\mathrm{B}(5)$ & $138.1(3)$ \\
\hline $\mathrm{B}(2)-\mathrm{B}(6)-\mathrm{B}(11)-\mathrm{B}(5)$ & $100.6(3)$ \\
\hline $\mathrm{C}(1)-\mathrm{B}(6)-\mathrm{B}(11)-\mathrm{B}(12)$ & $-62.2(4)$ \\
\hline $\mathrm{B}(7)-\mathrm{B}(6)-\mathrm{B}(11)-\mathrm{B}(12)$ & $37.5(3)$ \\
\hline $\mathrm{B}(2)-\mathrm{B}(6)-\mathrm{B}(11)-\mathrm{B}(12)$ & $0.0(4)$ \\
\hline $\mathrm{B}(5)-\mathrm{B}(6)-\mathrm{B}(11)-\mathrm{B}(12)$ & $-100.6(4)$ \\
\hline $\mathrm{C}(1)-\mathrm{B}(6)-\mathrm{B}(11)-\mathrm{B}(7)$ & $-99.7(3)$ \\
\hline $\mathrm{B}(2)-\mathrm{B}(6)-\mathrm{B}(11)-\mathrm{B}(7)$ & $-37.5(3)$ \\
\hline $\mathrm{B}(5)-\mathrm{B}(6)-\mathrm{B}(11)-\mathrm{B}(7)$ & $-138.1(3)$ \\
\hline $\mathrm{C}(1)-\mathrm{B}(6)-\mathrm{B}(11)-\mathrm{B}(10)$ & $1.2(4)$ \\
\hline $\mathrm{B}(7)-\mathrm{B}(6)-\mathrm{B}(11)-\mathrm{B}(10)$ & $100.9(3)$ \\
\hline $\mathrm{B}(2)-\mathrm{B}(6)-\mathrm{B}(11)-\mathrm{B}(10)$ & $63.4(4)$ \\
\hline
\end{tabular}




\begin{tabular}{|c|c|}
\hline $\mathrm{B}(5)-\mathrm{B}(6)-\mathrm{B}(11)-\mathrm{B}(10)$ & $-37.2(3)$ \\
\hline $\mathrm{C}(1)-\mathrm{B}(5)-\mathrm{B}(11)-\mathrm{B}(6)$ & $-38.5(3)$ \\
\hline $\mathrm{B}(10)-\mathrm{B}(5)-\mathrm{B}(11)-\mathrm{B}(6)$ & $-138.3(3)$ \\
\hline $\mathrm{B}(4)-\mathrm{B}(5)-\mathrm{B}(11)-\mathrm{B}(6)$ & $-100.5(3)$ \\
\hline $\mathrm{C}(1)-\mathrm{B}(5)-\mathrm{B}(11)-\mathrm{B}(12)$ & $62.3(4)$ \\
\hline $\mathrm{B}(10)-\mathrm{B}(5)-\mathrm{B}(11)-\mathrm{B}(12)$ & $-37.6(3)$ \\
\hline $\mathrm{B}(6)-\mathrm{B}(5)-\mathrm{B}(11)-\mathrm{B}(12)$ & $100.8(3)$ \\
\hline $\mathrm{B}(4)-\mathrm{B}(5)-\mathrm{B}(11)-\mathrm{B}(12)$ & $0.3(4)$ \\
\hline $\mathrm{C}(1)-\mathrm{B}(5)-\mathrm{B}(11)-\mathrm{B}(7)$ & $-1.0(4)$ \\
\hline $\mathrm{B}(10)-\mathrm{B}(5)-\mathrm{B}(11)-\mathrm{B}(7)$ & $-100.9(3)$ \\
\hline $\mathrm{B}(6)-\mathrm{B}(5)-\mathrm{B}(11)-\mathrm{B}(7)$ & $37.5(3)$ \\
\hline $\mathrm{B}(4)-\mathrm{B}(5)-\mathrm{B}(11)-\mathrm{B}(7)$ & $-63.0(4)$ \\
\hline $\mathrm{C}(1)-\mathrm{B}(5)-\mathrm{B}(11)-\mathrm{B}(10)$ & $99.8(3)$ \\
\hline $\mathrm{B}(6)-\mathrm{B}(5)-\mathrm{B}(11)-\mathrm{B}(10)$ & $138.3(3)$ \\
\hline $\mathrm{B}(4)-\mathrm{B}(5)-\mathrm{B}(11)-\mathrm{B}(10)$ & $37.9(3)$ \\
\hline $\mathrm{B}(8)-\mathrm{B}(7)-\mathrm{B}(11)-\mathrm{B}(6)$ & $100.7(3)$ \\
\hline $\mathrm{B}(2)-\mathrm{B}(7)-\mathrm{B}(11)-\mathrm{B}(6)$ & $37.3(3)$ \\
\hline $\mathrm{B}(12)-\mathrm{B}(7)-\mathrm{B}(11)-\mathrm{B}(6)$ & $138.0(3)$ \\
\hline $\mathrm{B}(6)-\mathrm{B}(7)-\mathrm{B}(11)-\mathrm{B}(5)$ & $-37.6(3)$ \\
\hline $\mathrm{B}(8)-\mathrm{B}(7)-\mathrm{B}(11)-\mathrm{B}(5)$ & $63.1(4)$ \\
\hline $\mathrm{B}(2)-\mathrm{B}(7)-\mathrm{B}(11)-\mathrm{B}(5)$ & $-0.3(4)$ \\
\hline $\mathrm{B}(12)-\mathrm{B}(7)-\mathrm{B}(11)-\mathrm{B}(5)$ & $100.4(3)$ \\
\hline $\mathrm{B}(6)-\mathrm{B}(7)-\mathrm{B}(11)-\mathrm{B}(12)$ & $-138.0(3)$ \\
\hline $\mathrm{B}(8)-\mathrm{B}(7)-\mathrm{B}(11)-\mathrm{B}(12)$ & $-37.2(3)$ \\
\hline $\mathrm{B}(2)-\mathrm{B}(7)-\mathrm{B}(11)-\mathrm{B}(12)$ & $-100.6(4)$ \\
\hline $\mathrm{B}(6)-\mathrm{B}(7)-\mathrm{B}(11)-\mathrm{B}(10)$ & $-100.8(3)$ \\
\hline B(8)-B(7)-B(11)-B(10) & $0.0(4)$ \\
\hline $\mathrm{B}(2)-\mathrm{B}(7)-\mathrm{B}(11)-\mathrm{B}(10)$ & $-63.4(4)$ \\
\hline $\mathrm{B}(12)-\mathrm{B}(7)-\mathrm{B}(11)-\mathrm{B}(10)$ & $37.2(3)$ \\
\hline $\mathrm{B}(4)-\mathrm{B}(10)-\mathrm{B}(11)-\mathrm{B}(6)$ & $-0.8(4)$ \\
\hline $\mathrm{B}(5)-\mathrm{B}(10)-\mathrm{B}(11)-\mathrm{B}(6)$ & $37.4(3)$ \\
\hline $\mathrm{B}(12)-\mathrm{B}(10)-\mathrm{B}(11)-\mathrm{B}(6)$ & $-100.5(3)$ \\
\hline $\mathrm{B}(9)-\mathrm{B}(10)-\mathrm{B}(11)-\mathrm{B}(6)$ & $-63.5(3)$ \\
\hline $\mathrm{B}(4)-\mathrm{B}(10)-\mathrm{B}(11)-\mathrm{B}(5)$ & $-38.2(3)$ \\
\hline $\mathrm{B}(12)-\mathrm{B}(10)-\mathrm{B}(11)-\mathrm{B}(5)$ & $-137.9(3)$ \\
\hline $\mathrm{B}(9)-\mathrm{B}(10)-\mathrm{B}(11)-\mathrm{B}(5)$ & $-100.9(3)$ \\
\hline
\end{tabular}




\begin{tabular}{|c|c|}
\hline $\mathrm{B}(4)-\mathrm{B}(10)-\mathrm{B}(11)-\mathrm{B}(12)$ & $99.7(3)$ \\
\hline $\mathrm{B}(5)-\mathrm{B}(10)-\mathrm{B}(11)-\mathrm{B}(12)$ & $137.9(3)$ \\
\hline $\mathrm{B}(9)-\mathrm{B}(10)-\mathrm{B}(11)-\mathrm{B}(12)$ & $37.0(3)$ \\
\hline $\mathrm{B}(4)-\mathrm{B}(10)-\mathrm{B}(11)-\mathrm{B}(7)$ & $62.6(4)$ \\
\hline $\mathrm{B}(5)-\mathrm{B}(10)-\mathrm{B}(11)-\mathrm{B}(7)$ & $100.7(3)$ \\
\hline $\mathrm{B}(12)-\mathrm{B}(10)-\mathrm{B}(11)-\mathrm{B}(7)$ & $-37.2(3)$ \\
\hline $\mathrm{B}(9)-\mathrm{B}(10)-\mathrm{B}(11)-\mathrm{B}(7)$ & $-0.1(4)$ \\
\hline $\mathrm{B}(4)-\mathrm{B}(9)-\mathrm{B}(12)-\mathrm{B}(8)$ & $100.7(3)$ \\
\hline $\mathrm{B}(3)-\mathrm{B}(9)-\mathrm{B}(12)-\mathrm{B}(8)$ & $37.2(3)$ \\
\hline $\mathrm{B}(10)-\mathrm{B}(9)-\mathrm{B}(12)-\mathrm{B}(8)$ & 138.1(3) \\
\hline $\mathrm{B}(4)-\mathrm{B}(9)-\mathrm{B}(12)-\mathrm{B}(7)$ & $63.6(4)$ \\
\hline $\mathrm{B}(3)-\mathrm{B}(9)-\mathrm{B}(12)-\mathrm{B}(7)$ & $0.2(4)$ \\
\hline $\mathrm{B}(8)-\mathrm{B}(9)-\mathrm{B}(12)-\mathrm{B}(7)$ & $-37.1(3)$ \\
\hline $\mathrm{B}(10)-\mathrm{B}(9)-\mathrm{B}(12)-\mathrm{B}(7)$ & 101.1(3) \\
\hline $\mathrm{B}(4)-\mathrm{B}(9)-\mathrm{B}(12)-\mathrm{B}(11)$ & $0.0(4)$ \\
\hline $\mathrm{B}(3)-\mathrm{B}(9)-\mathrm{B}(12)-\mathrm{B}(11)$ & $-63.4(4)$ \\
\hline $\mathrm{B}(8)-\mathrm{B}(9)-\mathrm{B}(12)-\mathrm{B}(11)$ & $-100.6(3)$ \\
\hline $\mathrm{B}(10)-\mathrm{B}(9)-\mathrm{B}(12)-\mathrm{B}(11)$ & $37.5(3)$ \\
\hline $\mathrm{B}(4)-\mathrm{B}(9)-\mathrm{B}(12)-\mathrm{B}(10)$ & $-37.5(3)$ \\
\hline $\mathrm{B}(3)-\mathrm{B}(9)-\mathrm{B}(12)-\mathrm{B}(10)$ & $-100.9(3)$ \\
\hline $\mathrm{B}(8)-\mathrm{B}(9)-\mathrm{B}(12)-\mathrm{B}(10)$ & $-138.1(3)$ \\
\hline $\mathrm{B}(3)-\mathrm{B}(8)-\mathrm{B}(12)-\mathrm{B}(9)$ & $-37.2(3)$ \\
\hline $\mathrm{B}(7)-\mathrm{B}(8)-\mathrm{B}(12)-\mathrm{B}(9)$ & $-138.5(3)$ \\
\hline $\mathrm{B}(2)-\mathrm{B}(8)-\mathrm{B}(12)-\mathrm{B}(9)$ & $-100.8(3)$ \\
\hline $\mathrm{B}(3)-\mathrm{B}(8)-\mathrm{B}(12)-\mathrm{B}(7)$ & 101.3(3) \\
\hline $\mathrm{B}(2)-\mathrm{B}(8)-\mathrm{B}(12)-\mathrm{B}(7)$ & $37.7(3)$ \\
\hline $\mathrm{B}(9)-\mathrm{B}(8)-\mathrm{B}(12)-\mathrm{B}(7)$ & $138.5(3)$ \\
\hline $\mathrm{B}(3)-\mathrm{B}(8)-\mathrm{B}(12)-\mathrm{B}(11)$ & $64.0(4)$ \\
\hline $\mathrm{B}(7)-\mathrm{B}(8)-\mathrm{B}(12)-\mathrm{B}(11)$ & $-37.3(3)$ \\
\hline $\mathrm{B}(2)-\mathrm{B}(8)-\mathrm{B}(12)-\mathrm{B}(11)$ & $0.4(4)$ \\
\hline $\mathrm{B}(9)-\mathrm{B}(8)-\mathrm{B}(12)-\mathrm{B}(11)$ & $101.2(3)$ \\
\hline $\mathrm{B}(3)-\mathrm{B}(8)-\mathrm{B}(12)-\mathrm{B}(10)$ & $0.4(4)$ \\
\hline $\mathrm{B}(7)-\mathrm{B}(8)-\mathrm{B}(12)-\mathrm{B}(10)$ & $-100.9(3)$ \\
\hline $\mathrm{B}(2)-\mathrm{B}(8)-\mathrm{B}(12)-\mathrm{B}(10)$ & $-63.2(4)$ \\
\hline $\mathrm{B}(9)-\mathrm{B}(8)-\mathrm{B}(12)-\mathrm{B}(10)$ & $37.7(3)$ \\
\hline $\mathrm{B}(6)-\mathrm{B}(7)-\mathrm{B}(12)-\mathrm{B}(9)$ & $-63.8(4)$ \\
\hline
\end{tabular}




\begin{tabular}{|c|c|}
\hline $\mathrm{B}(8)-\mathrm{B}(7)-\mathrm{B}(12)-\mathrm{B}(9)$ & $37.2(3)$ \\
\hline $\mathrm{B}(2)-\mathrm{B}(7)-\mathrm{B}(12)-\mathrm{B}(9)$ & $-0.5(4)$ \\
\hline $\mathrm{B}(11)-\mathrm{B}(7)-\mathrm{B}(12)-\mathrm{B}(9)$ & $-101.1(3)$ \\
\hline $\mathrm{B}(6)-\mathrm{B}(7)-\mathrm{B}(12)-\mathrm{B}(8)$ & $-101.0(3)$ \\
\hline $\mathrm{B}(2)-\mathrm{B}(7)-\mathrm{B}(12)-\mathrm{B}(8)$ & $-37.6(3)$ \\
\hline $\mathrm{B}(11)-\mathrm{B}(7)-\mathrm{B}(12)-\mathrm{B}(8)$ & $-138.3(3)$ \\
\hline $\mathrm{B}(6)-\mathrm{B}(7)-\mathrm{B}(12)-\mathrm{B}(11)$ & $37.3(3)$ \\
\hline $\mathrm{B}(8)-\mathrm{B}(7)-\mathrm{B}(12)-\mathrm{B}(11)$ & $138.3(3)$ \\
\hline $\mathrm{B}(2)-\mathrm{B}(7)-\mathrm{B}(12)-\mathrm{B}(11)$ & $100.6(3)$ \\
\hline $\mathrm{B}(6)-\mathrm{B}(7)-\mathrm{B}(12)-\mathrm{B}(10)$ & $0.0(4)$ \\
\hline $\mathrm{B}(8)-\mathrm{B}(7)-\mathrm{B}(12)-\mathrm{B}(10)$ & $101.0(3)$ \\
\hline $\mathrm{B}(2)-\mathrm{B}(7)-\mathrm{B}(12)-\mathrm{B}(10)$ & $63.4(4)$ \\
\hline $\mathrm{B}(11)-\mathrm{B}(7)-\mathrm{B}(12)-\mathrm{B}(10)$ & $-37.2(3)$ \\
\hline $\mathrm{B}(6)-\mathrm{B}(11)-\mathrm{B}(12)-\mathrm{B}(9)$ & $63.4(4)$ \\
\hline $\mathrm{B}(5)-\mathrm{B}(11)-\mathrm{B}(12)-\mathrm{B}(9)$ & $-0.2(4)$ \\
\hline $\mathrm{B}(7)-\mathrm{B}(11)-\mathrm{B}(12)-\mathrm{B}(9)$ & $100.8(3)$ \\
\hline $\mathrm{B}(10)-\mathrm{B}(11)-\mathrm{B}(12)-\mathrm{B}(9)$ & $-37.6(3)$ \\
\hline $\mathrm{B}(6)-\mathrm{B}(11)-\mathrm{B}(12)-\mathrm{B}(8)$ & $-0.2(4)$ \\
\hline $\mathrm{B}(5)-\mathrm{B}(11)-\mathrm{B}(12)-\mathrm{B}(8)$ & $-63.8(4)$ \\
\hline $\mathrm{B}(7)-\mathrm{B}(11)-\mathrm{B}(12)-\mathrm{B}(8)$ & $37.2(3)$ \\
\hline $\mathrm{B}(10)-\mathrm{B}(11)-\mathrm{B}(12)-\mathrm{B}(8)$ & $-101.2(3)$ \\
\hline $\mathrm{B}(6)-\mathrm{B}(11)-\mathrm{B}(12)-\mathrm{B}(7)$ & $-37.5(3)$ \\
\hline $\mathrm{B}(5)-\mathrm{B}(11)-\mathrm{B}(12)-\mathrm{B}(7)$ & $-101.0(3)$ \\
\hline $\mathrm{B}(10)-\mathrm{B}(11)-\mathrm{B}(12)-\mathrm{B}(7)$ & $-138.5(3)$ \\
\hline $\mathrm{B}(6)-\mathrm{B}(11)-\mathrm{B}(12)-\mathrm{B}(10)$ & $101.0(3)$ \\
\hline $\mathrm{B}(5)-\mathrm{B}(11)-\mathrm{B}(12)-\mathrm{B}(10)$ & $37.4(3)$ \\
\hline $\mathrm{B}(7)-\mathrm{B}(11)-\mathrm{B}(12)-\mathrm{B}(10)$ & $138.5(3)$ \\
\hline $\mathrm{B}(4)-\mathrm{B}(10)-\mathrm{B}(12)-\mathrm{B}(9)$ & $37.0(3)$ \\
\hline $\mathrm{B}(5)-\mathrm{B}(10)-\mathrm{B}(12)-\mathrm{B}(9)$ & $100.7(3)$ \\
\hline $\mathrm{B}(11)-\mathrm{B}(10)-\mathrm{B}(12)-\mathrm{B}(9)$ & $138.2(3)$ \\
\hline $\mathrm{B}(4)-\mathrm{B}(10)-\mathrm{B}(12)-\mathrm{B}(8)$ & $-0.5(4)$ \\
\hline $\mathrm{B}(5)-\mathrm{B}(10)-\mathrm{B}(12)-\mathrm{B}(8)$ & $63.1(4)$ \\
\hline $\mathrm{B}(11)-\mathrm{B}(10)-\mathrm{B}(12)-\mathrm{B}(8)$ & $100.6(4)$ \\
\hline $\mathrm{B}(9)-\mathrm{B}(10)-\mathrm{B}(12)-\mathrm{B}(8)$ & $-37.6(3)$ \\
\hline $\mathrm{B}(4)-\mathrm{B}(10)-\mathrm{B}(12)-\mathrm{B}(7)$ & $-63.9(4)$ \\
\hline $\mathrm{B}(5)-\mathrm{B}(10)-\mathrm{B}(12)-\mathrm{B}(7)$ & $-0.2(4)$ \\
\hline
\end{tabular}




\begin{tabular}{|c|c|}
\hline $\mathrm{B}(11)-\mathrm{B}(10)-\mathrm{B}(12)-\mathrm{B}(7)$ & $37.2(3)$ \\
\hline $\mathrm{B}(9)-\mathrm{B}(10)-\mathrm{B}(12)-\mathrm{B}(7)$ & $-100.9(3)$ \\
\hline $\mathrm{B}(4)-\mathrm{B}(10)-\mathrm{B}(12)-\mathrm{B}(11)$ & $-101.1(3)$ \\
\hline $\mathrm{B}(5)-\mathrm{B}(10)-\mathrm{B}(12)-\mathrm{B}(11)$ & $-37.5(3)$ \\
\hline $\mathrm{B}(9)-\mathrm{B}(10)-\mathrm{B}(12)-\mathrm{B}(11)$ & $-138.2(3)$ \\
\hline $\mathrm{C}(5)-\mathrm{P}(1)-\mathrm{C}(2)-\mathrm{C}(4)$ & $173.0(3)$ \\
\hline $\mathrm{C}(1)-\mathrm{P}(1)-\mathrm{C}(2)-\mathrm{C}(4)$ & $63.4(3)$ \\
\hline $\operatorname{Ir}(1)-\mathrm{P}(1)-\mathrm{C}(2)-\mathrm{C}(4)$ & $-61.5(4)$ \\
\hline $\mathrm{C}(5)-\mathrm{P}(1)-\mathrm{C}(2)-\mathrm{C}(3)$ & $-61.2(3)$ \\
\hline $\mathrm{C}(1)-\mathrm{P}(1)-\mathrm{C}(2)-\mathrm{C}(3)$ & $-170.8(3)$ \\
\hline $\operatorname{Ir}(1)-P(1)-C(2)-C(3)$ & $64.3(4)$ \\
\hline$C(2)-P(1)-C(5)-C(6)$ & $93.6(4)$ \\
\hline$C(1)-P(1)-C(5)-C(6)$ & $-156.3(4)$ \\
\hline $\operatorname{Ir}(1)-P(1)-C(5)-C(6)$ & $-39.1(4)$ \\
\hline $\mathrm{C}(2)-\mathrm{P}(1)-\mathrm{C}(5)-\mathrm{C}(7)$ & $-34.0(4)$ \\
\hline $\mathrm{C}(1)-\mathrm{P}(1)-\mathrm{C}(5)-\mathrm{C}(7)$ & $76.1(4)$ \\
\hline $\operatorname{Ir}(1)-\mathrm{P}(1)-\mathrm{C}(5)-\mathrm{C}(7)$ & $-166.8(3)$ \\
\hline $\mathrm{C}(4 \mathrm{~S})-\mathrm{O}(1 \mathrm{~S})-\mathrm{C}(1 \mathrm{~S})-\mathrm{C}(2 \mathrm{~S})$ & $-27.4(18)$ \\
\hline $\mathrm{O}(1 \mathrm{~S})-\mathrm{C}(1 \mathrm{~S})-\mathrm{C}(2 \mathrm{~S})-\mathrm{C}(3 \mathrm{~S})$ & 21.3(13) \\
\hline $\mathrm{C}(1 \mathrm{~S})-\mathrm{C}(2 \mathrm{~S})-\mathrm{C}(3 \mathrm{~S})-\mathrm{C}(4 \mathrm{~S})$ & $-7.8(14)$ \\
\hline $\mathrm{C}(1 \mathrm{~S})-\mathrm{O}(1 \mathrm{~S})-\mathrm{C}(4 \mathrm{~S})-\mathrm{C}(3 \mathrm{~S})$ & $23(2)$ \\
\hline $\mathrm{C}(2 \mathrm{~S})-\mathrm{C}(3 \mathrm{~S})-\mathrm{C}(4 \mathrm{~S})-\mathrm{O}(1 \mathrm{~S})$ & $-8.5(19)$ \\
\hline $\mathrm{C}(4 \mathrm{D})-\mathrm{O}(1 \mathrm{D})-\mathrm{C}(1 \mathrm{D})-\mathrm{C}(2 \mathrm{D})$ & $26(3)$ \\
\hline $\mathrm{O}(1 \mathrm{D})-\mathrm{C}(1 \mathrm{D})-\mathrm{C}(2 \mathrm{D})-\mathrm{C}(3 \mathrm{D})$ & $-19(3)$ \\
\hline $\mathrm{C}(1 \mathrm{D})-\mathrm{C}(2 \mathrm{D})-\mathrm{C}(3 \mathrm{D})-\mathrm{C}(4 \mathrm{D})$ & $4(2)$ \\
\hline $\mathrm{C}(1 \mathrm{D})-\mathrm{O}(1 \mathrm{D})-\mathrm{C}(4 \mathrm{D})-\mathrm{C}(3 \mathrm{D})$ & $-23(3)$ \\
\hline $\mathrm{C}(2 \mathrm{D})-\mathrm{C}(3 \mathrm{D})-\mathrm{C}(4 \mathrm{D})-\mathrm{O}(1 \mathrm{D})$ & $12(3)$ \\
\hline
\end{tabular}

Symmetry transformations used to generate equivalent atoms: 


\section{References:}

1. El-Hellani, A., Kefalidis, C.E., Tham, F.S., Maron, L., Lavallo, V. Organometallics. 2013, 32 (23), 6887.

2. Nunez, R., Vinas, C., Teixidor, F., Sillanpaa, R., Kivekas, R. J. Organomet. Chem. 1999, 592, 22.

3. APEX 2, version 2014.1-1, Bruker (2014), Bruker AXS Inc., Madison, Wisconsin, USA.

4. SAINT, version V8.34A, Bruker (2012), Bruker AXS Inc., Madison, Wisconsin, USA.

5. SADABS, version 2012/1, Bruker (2012), Bruker AXS Inc., Madison, Wisconsin, USA.

6. SHELXTL, version 2013/4, Bruker (2013), Bruker AXS Inc., Madison, Wisconsin, USA. 